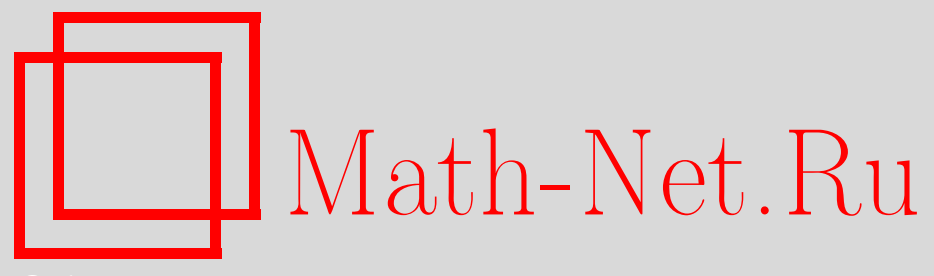

В. В. Бавула, Алгебры Ли треугольных полиномиальных дифференцирований и критерий изоморфности их факторалгебр Ли, Изв. РАН. Сер. матем., 2013, том 77, выпуск $6,3-44$

DOI: https://doi.org/10.4213/im8005

Использование Общероссийского математического портала Math-Net.Ru подразумевает, что вы прочитали и согласны с пользовательским соглашением http: //www . mathnet.ru/rus/agreement

Параметры загрузки:

IP: 52.90 .164 .192

26 апреля 2023 г., 04:14:57

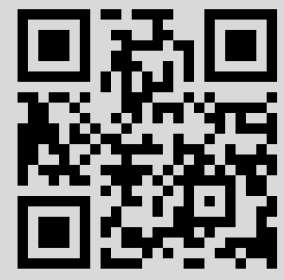




\section{В. В. Бавула}

\section{Алгебры Ли \\ треугольных полиномиальных дифференцирований и критерий изоморфности их факторалгебр Ли}

Подробно изучаются алгебры Ли $\mathfrak{u}_{n}, n \geqslant 2$, треугольных полиномиальных дифференцирований, их прямой предел $\mathfrak{u}_{\infty}$ и пополнение $\widehat{\mathfrak{u}}_{\infty}$. Классифицируются идеалы алгебр $\mathfrak{u}_{n}$, все они являются характеристическими идеалами. С помощью классификации идеалов дается явный критерий изоморфности факторалгебр Ли алгебр $\mathfrak{u}_{n}$ и $\mathfrak{u}_{m}$. Для алгебр (Ли) и их модулей вводятся две новые размерности: центральная размерность с. dim и однорядная размерность $\mathrm{u} . \operatorname{dim}$. Показано, что с. $\operatorname{dim}\left(\mathfrak{u}_{n}\right)=\mathrm{u} \cdot \operatorname{dim}\left(\mathfrak{u}_{n}\right)=$ $\omega^{n-1}+\omega^{n-2}+\cdots+\omega+1$ для всех $n \geqslant 2$, где $\omega-$ первый бесконечный ординал. Аналогичные результаты доказываются для алгебр Ли $\mathfrak{u}_{\infty}$ и $\widehat{\mathfrak{u}}_{\infty}$. В частности, $\mathrm{u} \cdot \operatorname{dim}\left(\mathfrak{u}_{\infty}\right)=\omega^{\omega}$ и с. $\operatorname{dim}\left(\mathfrak{u}_{\infty}\right)=0$.

Библиография: 5 наименований.

Ключевые слова: алгебра Ли, треугольные полиномиальные дифференцирования, автоморфизм, проблема изоморфизма, ряд коммутантов, нижний центральный ряд, локально нильпотентное дифференцирование, локально нильпотентная и локально конечномерная алгебра Ли.

DOI: $10.4213 / \operatorname{im} 8005$

\section{§ 1. Введение}

Всюду далее модуль означает левый модуль; $\mathbb{N}:=\{0,1, \ldots\}-$ множество натуральных чисел; $K$ - поле характеристики нуль, $K^{*}$ - его группа единиц; $P_{n}:=$ $K\left[x_{1}, \ldots, x_{n}\right]=\bigoplus_{\alpha \in \mathbb{N}^{n}} K x^{\alpha}-$ алгебра многочленов над $K$, где $x^{\alpha}:=x_{1}^{\alpha_{1}} \cdots x_{n}^{\alpha_{n}}$; $\partial_{1}:=\frac{\partial}{\partial x_{1}}, \ldots, \partial_{n}:=\frac{\partial}{\partial x_{n}}-$ частные производные $(K$-линейные дифференцирования) алгебры $P_{n} ; \operatorname{Der}_{K}\left(P_{n}\right)=\bigoplus_{i=1}^{n} P_{n} \partial_{i}$ - алгебра Ли $K$-дифференцирований алгебры $P_{n} ; A_{n}:=K\left\langle x_{1}, \ldots, x_{n}, \partial_{1}, \ldots, \partial_{n}\right\rangle=\bigoplus_{\alpha, \beta \in \mathbb{N}^{n}} K x^{\alpha} \partial^{\beta}-n$-я алгебра Вейля; для каждого натурального $n \geqslant 2$

$$
\mathfrak{u}_{n}:=K \partial_{1}+P_{1} \partial_{2}+\cdots+P_{n-1} \partial_{n}
$$

- алгебра Ли треугольных полиномиальных дифферениирований (это подалгебра Ли алгебры Ли $\left.\operatorname{Der}_{K}\left(P_{n}\right)\right), U_{n}:=U\left(\mathfrak{u}_{n}\right)$ - ее универсальная обертывающая алгебра.

Многие свойства алгебр Ли $\mathfrak{u}_{n}$, где $n \geqslant 2$, доказаны в предложении 2.1. Доказано, в частности, что алгебры Ли $\mathfrak{u}_{n}, n \geqslant 2$, попарно неизоморфны, все они являются разрешимыми, но не нильпотентными алгебрами Ли, а их внутренние

Работа выполнена при частичной поддержка Королевского общества (Royal Society) и Исследовательского совета по инженерным и физическим наукам (EPSRC). 
дифференцирования являются локально нильпотентными дифференцированиями. Найдены ряд коммутантов и нижний центральный ряд алгебры Ли $\mathfrak{u}_{n}$.

Вводится новая размерность для алгебр и модулей - однорядная размерность (см. §4), которая оказалась очень полезным инструментом в изучении ненётеровых алгебр Ли, их идеалов и автоморфизмов [1], [2].

В $\S 3$ дается классификация всех идеалов алгебры $\mathfrak{u}_{n}$ и для каждого идеала указывается явный базис. Доказывается, что $\mathfrak{u}_{n}$ является однорядной, артиновой, но не нётеровой алгеброй Ли, а ее однорядная размерность есть

$$
\text { u. } \operatorname{dim}\left(\mathfrak{u}_{n}\right)=\omega^{n-1}+\omega^{n-2}+\cdots+\omega+1,
$$

где $\omega$ - первый бесконечный ординал (теорема 3.6). Показано, что гиперцентральный ряд $\left\{Z^{(\lambda)}\left(\mathfrak{u}_{n}\right)\right\}_{\lambda \in \mathrm{W}}$ стабилизируется в точности на шаге $\operatorname{ord}\left(\Omega_{n}\right):=$ $\omega^{n-1}+\omega^{n-2}+\cdots+\omega+1$. Более того, $Z^{(\lambda)}\left(\mathfrak{u}_{n}\right)=I_{\lambda}$ для каждого $\lambda \in\left[1, \operatorname{ord}\left(\Omega_{n}\right)\right]$, где $I_{\lambda}$ явно задано формулой (3.3). Центральная размерность алгебры Ли $\mathfrak{u}_{n}$ есть с. $\operatorname{dim}\left(\mathfrak{u}_{n}\right)=\operatorname{ord}\left(\Omega_{n}\right)$ (теорема 3.10). Доказано, что все идеалы алгебры Ли $\mathfrak{u}_{n}$ являются характеристическими идеалами (следствие 3.11 ).

Алгебра Ли $\mathcal{G}$ называется локально нильпотентной (соответственно, локально конечномерной) алгеброй Ли, если каждая конечно порожденная подалгебра Ли алгебры $\mathcal{G}$ является нильпотентной (соответственно, конечномерной) алгеброй Ли. В $\S 4$ доказано, что все алгебры Ли $\mathfrak{u}_{n}$ являются локально конечномерными и локально нильпотентными (теорема 4.2).

В $§ 5$ теорема 5.1 и следствие 5.2 дают ответ на следующий

Вопрос. Пусть $I$ и $J$ - идеалы алгебр Ли $\mathfrak{u}_{n}$ и $\mathfrak{u}_{m}$ соответственно. При каких условиях факторалгебры Ли $\mathfrak{u}_{n} / I$ и $\mathfrak{u}_{m} / J$ изоморфны?

Ответ дается в явном виде (через однорядные размерности идеалов $I$ и $J$ ) с использованием классификации идеалов алгебр Ли $\mathfrak{u}_{n}$ (теорема 3.6). В частности, имеется только счетное число идеалов $I$ алгебры Ли $\mathfrak{u}_{n}$, для которых $\mathfrak{u}_{n} / I \simeq \mathfrak{u}_{n}$. Теорема $\left.3.6,1\right)$ показывает, что каждый идеал $I$ алгебры Ли $\mathfrak{u}_{n}$ однозначно определяется его однорядной размерностью

$$
\lambda=\mathrm{u} \cdot \operatorname{dim}(I) \in\left[0, \omega^{n-1}+\omega^{n-2}+\cdots+\omega+1\right],
$$

т. е. $I=I_{\lambda}$, где $\omega-$ первый бесконечный ординал.

В $\S 6$ изучается алгебра Ли $\mathfrak{u}_{\infty}:=\bigcup_{n \geqslant 2} \mathfrak{u}_{n}=\bigoplus_{n \geqslant 2} P_{n-1} \partial_{n}$. Многие свойства алгебры Ли $\mathfrak{u}_{\infty}$ схожи со свойством алгебр $\mathfrak{u}_{n}, n \geqslant 2$, но существует ряд отличий. Например, алгебра Ли $\mathfrak{u}_{\infty}$ не разрешима, не артинова, но почти артинова, $\boldsymbol{u} \cdot \operatorname{dim}\left(\mathfrak{u}_{\infty}\right)=\omega^{\omega}$. Получена классификация всех идеалов алгебры Ли $\mathfrak{u}_{\infty}$ (теорема 6.2). Все идеалы алгебры Ли $\mathfrak{u}_{\infty}$ являются характеристическими идеалами (следствие 6.4). Следствие 6.3 дает явный критерий изоморфности двух факторалгебр Ли алгебры $\mathfrak{u}_{\infty}$.

В $\S 7$ изучается топологическая алгебра Ли $\widehat{\mathfrak{u}}_{\infty}$, являющаяся пополнением алгебры Ли $\mathfrak{u}_{\infty}$. Ее свойства еще далее отклоняются от свойств алгебр Ли $\mathfrak{u}_{n}$, $n \geqslant 2$, и $\mathfrak{u}_{\infty}$. Классифицируются все замкнутые и все открытые идеалы топологической алгебры Ли $\widehat{\mathfrak{u}}_{\infty}$ (теорема $\left.7.2,1\right)$ ). 


\section{§ 2. Ряд коммутантов и нижний центральный ряд алгебр Ли $\mathfrak{u}_{n}$}

В этом параграфе доказываются различные свойства алгебр Ли $\mathfrak{u}_{n}$ (предложение 2.1, следствие 2.3), которые широко используются в настоящей работе. В конце параграфа вычисляются образ и ядро гомоморфизма алгебр $\chi_{n}: U_{n} \rightarrow A_{n-1} \otimes K\left[\partial_{n}\right]$ (см. (2.11) и теорему 2.6). В частности, показывается, что алгебра $\chi_{n}\left(U_{n}\right)$ не конечно порождена и не является ни лево-, ни правонётеровой.

Пусть $\mathcal{G}$ - алгебра Ли над полем $K$, a $\mathfrak{a}, \mathfrak{b}$ - ее идеалы. Kоммутант $[\mathfrak{a}, \mathfrak{b}]$ идеалов $\mathfrak{a}$ и $\mathfrak{b}-$ это линейная оболочка в $\mathcal{G}$ всех элементов $[a, b]$, где $a \in \mathfrak{a}$ и $b \in \mathfrak{b}$. Коммутант $[U, V]$ подпространств $U$ и $V$ алгебры $\mathcal{G}$ определяется таким же образом. Коммутант $[\mathfrak{a}, \mathfrak{b}]$ является идеалом алгебры Ли $\mathcal{G}$, для которого $[\mathfrak{a}, \mathfrak{b}] \subseteq \mathfrak{a} \cap \mathfrak{b}$. В частности, $\mathcal{G}_{(1)}:=\mathcal{G}^{(1)}:=[\mathcal{G}, \mathcal{G}]$ называется коммутантом алгебры Ли $\mathcal{G}$. Определим по индукции следующее множество идеалов алгебры Ли $\mathcal{G}$ :

$$
\mathcal{G}_{(i)}:=\left[\mathcal{G}_{(i-1)}, \mathcal{G}_{(i-1)}\right], \quad \mathcal{G}^{(i)}:=\left[\mathcal{G}, \mathcal{G}^{(i-1)}\right], \quad i \geqslant 2 .
$$

Ясно, что $\mathcal{G}_{(i)} \subseteq \mathcal{G}^{(i)}$ для всех $i \geqslant 1$. Убывающие цепочки идеалов алгебры Ли $\mathcal{G}$

$$
\begin{aligned}
& \mathcal{G}_{(0)}:=\mathcal{G} \supseteq \mathcal{G}_{(1)} \supseteq \cdots \supseteq \mathcal{G}_{(i)} \supseteq \mathcal{G}_{(i+1)} \supseteq \cdots, \\
& \mathcal{G}^{(0)}:=\mathcal{G} \supseteq \mathcal{G}^{(1)} \supseteq \cdots \supseteq \mathcal{G}^{(i)} \supseteq \mathcal{G}^{(i+1)} \supseteq \cdots
\end{aligned}
$$

называются рядом коммутантов и нижним центральным рядом алгебры Ли $\mathcal{G}$ соответственно. Заметим, что

$$
\mathfrak{u}_{n}=\bigoplus_{i=1}^{n} \bigoplus_{\alpha \in \mathbb{N}^{i-1}} K x^{\alpha} \partial_{i}
$$

Значит, элементы

$$
X_{\alpha, i}:=x^{\alpha} \partial_{i}=x_{1}^{\alpha_{1}} \cdots x_{i-1}^{\alpha_{i-1}} \partial_{i}, \quad i=1, \ldots, n, \quad \alpha \in \mathbb{N}^{i-1},
$$

образуют $K$-базис $\mathcal{B}_{n}$ алгебры Ли $\mathfrak{u}_{n}$. Базис $\mathcal{B}_{n}$ называется каноническим базисом алгебры $\mathfrak{u}_{n}$. Для всех $1 \leqslant i \leqslant j \leqslant n, \alpha \in \mathbb{N}^{i-1}$ и $\beta \in \mathbb{N}^{j-1}$ имеем

$$
\left[X_{\alpha, i}, X_{\beta, j}\right]=\left\{\begin{array}{lll}
0, & \text { если } & i=j, \\
\beta_{i} X_{\alpha+\beta-e_{i}, j}, & \text { если } & i<j,
\end{array}\right.
$$

где $e_{1}:=(1,0, \ldots, 0), \ldots, e_{n}:=(0, \ldots, 0,1)$ - канонический свободный $\mathbb{Z}$-базис $\mathbb{Z}$-модуля $\mathbb{Z}^{n}$ и $\mathbb{N}^{i}:=\sum_{k=1}^{i} \mathbb{N}_{k} \subseteq \mathbb{Z}^{i}:=\sum_{k=1}^{i} \mathbb{Z} e_{k}$. В частности, $\mathbb{N} \subseteq \mathbb{N}^{2} \subseteq \cdots$ $\cdots \subseteq \mathbb{N}^{n}$ и $\mathbb{Z} \subseteq \mathbb{Z}^{2} \subseteq \cdots \subseteq \mathbb{Z}^{n}$. Алгебра Ли $\mathfrak{u}_{n}=\bigoplus_{i=1}^{n} P_{i-1} \partial_{i}$ является прямой суммой абелевых (бесконечномерных, когда $i>1$ ) подалгебр Ли $P_{i-1} \partial_{i}$ (т. е. $\left.\left[P_{i-1} \partial_{i}, P_{i-1} \partial_{i}\right]=0\right)$ таких, что для всех $i<j$ выполнено

$$
\left[P_{i-1} \partial_{i}, P_{j-1} \partial_{j}\right]=P_{j-1} \partial_{j} .
$$

Вложение $\subseteq$ в $(2.3)$ очевидно, но равенство следует из того, что $\left[\partial_{i}, P_{j-1}\right]=$ $P_{j-1}$. В силу (2.3) алгебра Ли $\mathfrak{u}_{n}$ допускает конечную строго убывающую цепочку идеалов

$$
\mathfrak{u}_{n, 1}:=\mathfrak{u}_{n} \supset \mathfrak{u}_{n, 2} \supset \cdots \supset \mathfrak{u}_{n, i} \supset \cdots \supset \mathfrak{u}_{n, n} \supset \mathfrak{u}_{n, n+1}:=0,
$$


где $\mathfrak{u}_{n, i}:=\sum_{j=i}^{n} P_{j-1} \partial_{j}$ при $i=1, \ldots, n$. В силу $(2.3)$ для всех $i<j$

$$
\left[\mathfrak{u}_{n, i}, \mathfrak{u}_{n, j}\right] \subseteq\left\{\begin{array}{lll}
\mathfrak{u}_{n, i+1}, & \text { если } & i=j \\
\mathfrak{u}_{n, j}, & \text { если } & i<j
\end{array}\right.
$$

Для всех $i=1, \ldots, n$ имеется канонический изоморфизм алгебр Ли

$$
\mathfrak{u}_{i} \simeq \mathfrak{u}_{n} / \mathfrak{u}_{n, i+1}, \quad X_{\alpha, j} \mapsto X_{\alpha, j}+\mathfrak{u}_{n, i+1}
$$

В частности, $\mathfrak{u}_{n-1} \simeq \mathfrak{u}_{n} / P_{n-1} \partial_{n}$. Ясно, что

$$
\mathfrak{u}_{2} \subset \mathfrak{u}_{3} \subset \cdots \subset \mathfrak{u}_{n} \subset \mathfrak{u}_{n+1} \subset \cdots \subset \mathfrak{u}_{\infty}:=\bigcup_{n \geqslant 2} \mathfrak{u}_{n}=\bigoplus_{i \geqslant 1} \bigoplus_{\alpha \in \mathbb{N}^{i-1}} K x^{\alpha} \partial_{i}
$$

является возрастающей цепочкой алгебр Ли. Алгебра многочленов $P_{n}$ является $A_{n}$-модулем: для всех элементов $p \in P_{n}$

$$
x_{i} * p=x_{i} p, \quad \partial_{i} * p=\frac{\partial p}{\partial x_{i}}, \quad i=1, \ldots, n .
$$

Ясно, что $P_{n} \simeq A_{n} / \sum_{i=1}^{n} A_{n} \partial_{i}, 1 \mapsto 1+\sum_{i=1}^{n} A_{n} \partial_{i}$. Поскольку $\mathfrak{u}_{n} \subseteq A_{n}$, алгебра многочленов $P_{n}$ также является $\mathfrak{u}_{n}$-модулем.

Пусть $V$ - векторное пространство над полем $K$; $K$-линейное отображение $\delta: V \rightarrow V$ называется локально нильпотентным отображением, если $V=\bigcup_{i \geqslant 1} \operatorname{ker}\left(\delta^{i}\right)$, или, что равносильно, для каждого $v \in V$ выполняется $\delta^{i}(v)=0$ для всех $i \gg 1$. Когда $\delta$ - локально нильпотентное отображение в $V$, будем также говорить, что $\delta$ действует локально нильпотентно на $V$. Каждое нилъпотентное линейное отображение $\delta$, т. е. такое, что $\delta^{n}=0$ для некоторого $n \geqslant 1$, является локально нильпотентным отображением, но, вообще говоря, не наоборот. Пусть $\mathcal{G}$ - алгебра Ли. Каждый элемент $a \in \mathcal{G}$ определяет дифференцирование алгебры Ли $\mathcal{G}$ по правилу $\operatorname{ad}(a): \mathcal{G} \rightarrow \mathcal{G}, b \mapsto[a, b]$, которое называется внутренним дифберенцированием, связанным с $а$. Множество $\operatorname{Inn}(\mathcal{G})$ всех внутренних дифференцирований алгебры Ли $\mathcal{G}$ является подалгеброй Ли алгебры Ли $\left(\operatorname{End}_{K}(\mathcal{G}),[\cdot, \cdot]\right)$, где $[f, g]:=f g-g f$. Имеется короткая точная последовательность алгебр Ли

$$
0 \rightarrow Z(\mathcal{G}) \rightarrow \mathcal{G} \stackrel{\text { ad }}{\rightarrow} \operatorname{Inn}(\mathcal{G}) \rightarrow 0
$$

т. е. $\operatorname{Inn}(\mathcal{G}) \simeq \mathcal{G} / Z(\mathcal{G})$, где $Z(\mathcal{G})$ - центр алгебры Ли $\mathcal{G}$ и $\operatorname{ad}([a, b])=[\operatorname{ad}(a), \operatorname{ad}(b)]$ для всех элементов $a, b \in \mathcal{G}$. Элемент $a \in \mathcal{G}$ называется локально нильпотентным элементом (соответственно, нильпотентным элементом), если таковым является внутреннее дифференцирование $\operatorname{ad}(a)$ алгебры Ли $\mathcal{G}$. Пусть $J-$ непустое подмножество алгебры $\mathcal{G}$; тогда $\operatorname{Cen}_{\mathcal{G}}(J):=\{a \in \mathcal{G} \mid[a, b]=0$ для всех $b \in J\}$ называется централизатором $J$ в $\mathcal{G}$. Это подалгебра Ли алгебры Ли $\mathcal{G}$. Пусть $A$ - ассоциативная алгебра и $I-$ непустое подмножество из $A$. Тогда $\operatorname{Cen}_{A}(I):=\{a \in A \mid a b=b a$ для всех $b \in I\}$ называется иентрализатором $I$ в $A$. Это подалгебра из $A$. 
ПРЕДЛОЖЕНИЕ 2.1. 1) Алгебра Ли $\mathfrak{u}_{n}$ является разрешимой, но не нильпотентной алгеброй Ли.

2) Конечная цепочка идеалов (2.4) является рядом коммутантов алгебры Ли $\mathfrak{u}_{n}$, m.e. $\left(\mathfrak{u}_{n}\right)_{(i)}=\mathfrak{u}_{n, i+1}$ для всех $i \geqslant 0$.

3) Нижний центральный ряд алгебры Ли $\mathfrak{u}_{n}$ стабилизируется на первом maгe, m.e. $\left(\mathfrak{u}_{n}\right)^{(0)}=\mathfrak{u}_{n} u\left(\mathfrak{u}_{n}\right)^{(i)}=\mathfrak{u}_{n, 2}$ для всех $i \geqslant 1$.

4) Каждый элемент $u \in \mathfrak{u}_{n}$ действует локально нильпотентно на $\mathfrak{u}_{n}$-модуле $P_{n}$.

5) Все внутренние дифферениирования алгебры Ли $\mathfrak{u}_{n}$ являются локально нильпотентными дифференцированиями.

6) Центр $Z\left(\mathfrak{u}_{n}\right)$ алгебры Ли $\mathfrak{u}_{n}$ равен $K \partial_{n}$.

7) Алгебры Ли $\mathfrak{u}_{n}$, где $n \geqslant 2$, попарно неизоморфиъ.

ДоказАтельство. Утверждение 1) следует из утверждений 2) и 3), которые, в свою очередь, следуют из разложения $\mathfrak{u}_{n}=\bigoplus_{i=1}^{n} P_{i-1} \partial_{i}$ и $(2.3)$. Утверждение 4) следует из определения алгебр Ли $\mathfrak{u}_{n}$.

Докажем утверждение 5). Пусть $u \in \mathfrak{u}_{n, i} \backslash \mathfrak{u}_{n, i+1}$ для некоторого $i=1, \ldots, n$. Тогда $u=a \partial_{i}+u^{\prime}$ для некоторых элементов $a \in P_{i-1}$ и $u^{\prime} \in \mathfrak{u}_{n, i+1}$. Пусть $\delta=\operatorname{ad}(u), \partial=\operatorname{ad}\left(a \partial_{i}\right)$ и $v \in \mathfrak{u}_{n}$. Необходимо показать, что $\delta^{s}(v)=0$ для всех $s \gg 1$. Дважды применяя (2.5), видим, что $\delta(v) \in \mathfrak{u}_{n, i}$ и $\delta^{2}(v) \in \mathfrak{u}_{n, i+1}$. Заменяя элемент $v$ на $\delta^{2}(v)$, без ограничения общности можно считать, что $v \in \mathfrak{u}_{n, i+1}$ и $v \neq 0$. Ввиду равенства $\mathfrak{u}_{n, i+1}=\bigoplus_{j=i+1}^{n} P_{j-1} \partial_{j}$ имеем $v=b \partial_{i+1}+v^{\prime}$ для некоторых элементов $0 \neq b \in P_{i}$ и $v^{\prime} \in \mathfrak{u}_{n, i+2}$. Для всех натуральных $s \geqslant 1$ в силу (2.5) имеем

$$
\delta^{s}(v) \equiv \delta^{s}\left(b \partial_{i+1}\right) \equiv \delta^{s}(b) \partial_{i+1} \equiv \partial^{s}(b) \partial_{i+1} \bmod \mathfrak{u}_{n, i+2}
$$

В силу утверждения 4) имеем $\partial^{s}(b)=0$ для всех $s \gg 1$. Тогда $\delta^{s}(v) \in \mathfrak{u}_{n, i+2}$ для всех $s \gg 1$. Аналогично, $\delta^{s}(v) \in \mathfrak{u}_{n, i+3}$ для всех $s \gg 1$. Применяя то же рассуждение несколько раз, видим, что $\delta^{s}(v) \in \mathfrak{u}_{n, n+1}=0$ для всех $s \gg 1$. Это означает, что $\delta$ - локально нильпотентное отображение, как и требуется.

Теперь докажем утверждение 6). Хорошо известно и легко показать, что

$$
\operatorname{Cen}_{A_{n}}\left(\partial_{1}, \ldots, \partial_{n}\right)=K\left[\partial_{1}, \ldots, \partial_{n}\right]
$$

Отсюда следует, что

$$
\operatorname{Cen}_{A_{n}}\left(\partial_{1}, \ldots, \partial_{n}, x_{1} \partial_{n}, \ldots, x_{n-1} \partial_{n}\right)=K\left[\partial_{n}\right]
$$

а тогда

$$
Z\left(\mathfrak{u}_{n}\right) \subseteq \mathfrak{u}_{n} \cap \operatorname{Cen}_{A_{n}}\left(\partial_{1}, \ldots, \partial_{n}, x_{1} \partial_{n}, \ldots, x_{n-1} \partial_{n}\right)=\mathfrak{u}_{n} \cap K\left[\partial_{n}\right]=K \partial_{n}
$$

Обратное включение $Z\left(\mathfrak{u}_{n}\right) \supseteq K \partial_{n}$ очевидно. Значит, $Z\left(\mathfrak{u}_{n}\right)=K \partial_{n}$.

Утверждение 7) следует из утверждения 2), так как ряды коммутантов алгебр $\mathfrak{u}_{n}$ имеют различную длину (и являются инвариантами при изоморфизмах). Предложение доказано. 
Предложение $2.1,5)$ позволяет получить много автоморфизмов алгебры Ли $\mathfrak{u}_{n}$. Для каждого элемента $a \in \mathfrak{u}_{n}$ внутреннее дифференцирование $\operatorname{ad}(a)$ является локально нильпотентным дифференцированием, поэтому

$$
e^{\operatorname{ad}(a)}:=\sum_{i \geqslant 0} \frac{\operatorname{ad}(a)^{i}}{i !} \in \operatorname{Aut}_{K}\left(\mathfrak{u}_{n}\right) .
$$

B [1] найдены группа автоморфизмов $\operatorname{Aut}_{K}\left(\mathfrak{u}_{n}\right)$ алгебры Ли $\mathfrak{u}_{n}$ и ее явные порождающие и показано, что присоединенная группа $\left\langle e^{\operatorname{ad}(a)} \mid a \in \mathfrak{u}_{n}\right\rangle$ является очень малой частью группы $\operatorname{Aut}_{K}\left(\mathfrak{u}_{n}\right)$.

Следующая лемма классифицирует нильпотентные внутренние дифференцирования алгебр Ли $\mathfrak{u}_{n}$.

Лемма 2.2. Пусть $a \in \mathfrak{u}_{n} u \delta=\operatorname{ad}(a)$. Следующие утверждения равносильнь:

1) отображение $\delta$ является нильпотентным дифберениированием алгебры Лu $\mathfrak{u}_{n}$;

2) $a \in P_{n-1} \partial_{n}$;

3) $\delta^{2}=0$.

ДокАЗАтЕльство. Импликации 2) $\Rightarrow 3$ ) $\Rightarrow$ 1) очевидны.

Докажем импликацию 1) $\Rightarrow 2$ ). Покажем, что дифференцирование $\delta$ не является нильпотентным для каждого элемента $a \in \mathfrak{u}_{n} \backslash P_{n-1} \partial_{n}$. Пусть $u \in$ $\mathfrak{u}_{n} \backslash P_{n-1} \partial_{n}$, т. е. $u=p_{i} \partial_{i}+p_{i+1} \partial_{i+1}+\cdots+p_{n} \partial_{n}=p_{i} \partial_{i}+\cdots$, где $p_{j} \in P_{j-1}$ для всех $j=i, \ldots, n, i<n$, и $p_{i} \neq 0$. Поскольку

$$
\delta^{m}\left(x_{i}^{m} \partial_{i+1}\right)=m ! p_{i}^{m} \partial_{i+1}+\cdots, \quad m \geqslant 1,
$$

дифференцирование $\delta$ не является нильпотентным дифференцированием. Лемма доказана.

Пусть $A$ - кольцо. Подмножество $S$ из $A$ называется мультипликативным подмножеством или мультипликативно замкнутым подмножеством кольца $A$, если $1 \in S, S S \subseteq S$ и $0 \notin S$. Каждую ассоциативную алгебру $A$ можно

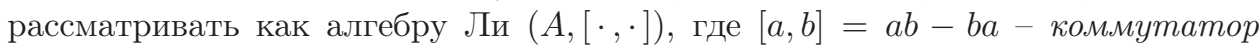
элементов $a, b \in A$. Для каждого элемента $a \in A$ отображение $\operatorname{ad}(a): A \rightarrow A$, $b \mapsto[a, b]$, является $K$-дифференцированием алгебры $A$, рассматриваемой как ассоциативная алгебра и алгебра Ли. Дифференцирование $\operatorname{ad}(a)$ называется внутренним дифференицрованием кольца $A$, связанным с элементом $a$. Таким образом, ассоциативная алгебра $A$ и алгебра Ли $(A,[\cdot, \cdot])$ имеют то же самое множество внутренних дифференцирований $\operatorname{Inn}(A)$ и тот же самый центр $Z(A)$.

Пусть $\delta$ - дифференцирование кольца $A$. Для всех элементов $a, b \in A$

$$
\begin{gathered}
\delta^{n}(a b)=\sum_{i=0}^{n}\left(\begin{array}{l}
n \\
i
\end{array}\right) \delta^{i}(a) \delta^{n-i}(b), \quad n \geqslant 1, \\
a^{n} b=\sum_{i=0}^{n}\left(\begin{array}{l}
n \\
i
\end{array}\right)(\operatorname{ad} a)^{i}(b) a^{n-i}, \quad n \geqslant 1 .
\end{gathered}
$$


СлеДСтвиЕ 2.3. 1) Внутренние дифберениирования $\left\{\operatorname{ad}(u) \mid u \in \mathfrak{u}_{n}\right\}$ универсальной обертывающей алгебры $U_{n}$ алгебры Ли $\mathfrak{u}_{n}$ являются локально нильпотентными дифферениированиями.

2) Каждое мультипликативное подмножество $S$ из $U_{n}$, порожденное произвольным множеством элементов алгебры $\mathfrak{u}_{n}$, является (левым и правым) множеством Оре в $U_{n}$. Значит, $S^{-1} U_{n} \simeq U_{n} S^{-1}$.

ДокАзАТЕльство. Утверждение 1) следует из (2.9) и предложения 2.1, 5). Утверждение 2) является несложным следствием из соотношения (2.10) и предложения $2.1,5)$. Следствие доказано.

Пример 2.4. Множество $S=\left\{\partial^{\alpha} \mid \alpha \in \mathbb{N}^{n}\right\}$ является мультипликативным подмножеством алгебры $U_{n}$. В силу следствия $\left.2.3,2\right)$ существует кольцо частных $S^{-1} U_{n}$.

Пусть $D$ - кольцо, $\sigma=\left(\sigma_{1}, \ldots, \sigma_{n}\right)$ - набор из $n$ коммутирующих автоморфизмов кольца $D$ (т. е. $\sigma_{i} \sigma_{j}=\sigma_{j} \sigma_{i}$ для всех $\left.i, j\right)$ и $a=\left(a_{1}, \ldots, a_{n}\right)$ - набор из $n$ (ненулевых) элементов центра $Z(D)$ кольца $D$, для которых $\sigma_{i}\left(a_{j}\right)=a_{j}$ при всех $i \neq j$.

Обобщенная алгебра Вейля $A=D(\sigma, a)$ (кратко ОАВ) степени $n$ с базовым кольцом $D$ - это кольцо, порожденное $D$ и $2 n$ переменными $x_{1}, \ldots, x_{n}$, $y_{1}, \ldots, y_{n}$, подчиненными следующим определяющим соотношениям [3], [4]:

$$
\begin{gathered}
y_{i} x_{i}=a_{i}, \quad x_{i} y_{i}=\sigma_{i}\left(a_{i}\right), \\
x_{i} \alpha=\sigma_{i}(\alpha) x_{i}, \quad y_{i} \alpha=\sigma_{i}^{-1}(\alpha) y_{i}, \quad \alpha \in D, \\
{\left[x_{i}, x_{j}\right]=\left[y_{i}, y_{j}\right]=\left[x_{i}, y_{j}\right]=0, \quad i \neq j,}
\end{gathered}
$$

где $[x, y]=x y-y x$. Будем говорить, что $a$ и $\sigma$ - множества определяющих элементов и автоморфизмов алгебры $A$ соответственно. Для вектора $k=\left(k_{1}, \ldots, k_{n}\right) \in \mathbb{Z}^{n}$ положим $v_{k}=v_{k_{1}}(1) \cdots v_{k_{n}}(n)$, где $v_{m}(i)=x_{i}^{m}$, $v_{-m}(i)=y_{i}^{m}, v_{0}(i)=1$ для $1 \leqslant i \leqslant n$ и $m \geqslant 0$. Из определения ОАВ следует, что

$$
A=\bigoplus_{k \in \mathbb{Z}^{n}} A_{k}
$$

является $\mathbb{Z}^{n}$-градуированной алгеброй $\left(A_{k} A_{e} \subset A_{k+e}\right.$ для всех $\left.k, e \in \mathbb{Z}^{n}\right)$, где $A_{k}=D v_{k}=v_{k} D$.

Пусть $\mathcal{P}_{n}$ - алгебра многочленов $K\left[H_{1}, \ldots, H_{n}\right]$ от $n$ переменных, и пусть $\sigma=\left(\sigma_{1}, \ldots, \sigma_{n}\right)$ - набор из $n$ коммутирующих автоморфизмов алгебры $\mathcal{P}_{n}$, определенный следующим образом: $\sigma_{i}\left(H_{i}\right)=H_{i}-1$ и $\sigma_{i}\left(H_{j}\right)=H_{j}$ при $i \neq j$. Отображение

$$
A_{n} \rightarrow \mathcal{P}_{n}\left(\left(\sigma_{1}, \ldots, \sigma_{n}\right),\left(H_{1}, \ldots, H_{n}\right)\right), \quad x_{i} \mapsto x_{i}, \quad \partial_{i} \mapsto y_{i}, \quad i=1, \ldots, n,
$$

является изоморфизмом $K$-алгебр. Мы отождествляем алгебру Вейля $A_{n}$ с вышеупомянутой ОАВ посредством этого изоморфизма. Алгебра Вейля $A_{n}=\bigoplus_{\alpha \in \mathbb{Z}^{n}} A_{n, \alpha}$ является $\mathbb{Z}$-градуированной алгеброй $\left(A_{n, \alpha} A_{n, \beta} \subseteq A_{n, \alpha+\beta}\right.$ для всех $\left.\alpha, \beta \in \mathbb{Z}^{n}\right)$. 
Мультипликативные множества $S=\left\{\partial^{\alpha} \mid \alpha \in \mathbb{N}^{n}\right\}$ и $T=\left\{x^{\alpha} \mid \alpha \in \mathbb{N}^{n}\right\}$ являются (левыми и правыми) множествами Оре алгебры Вейля $A_{n}$, причем

$$
\begin{gathered}
B_{n}:=S^{-1} A_{n}=K\left[H_{1}, \ldots, H_{n}\right]\left[\partial_{1}^{ \pm 1}, \ldots, \partial_{n}^{ \pm 1} ; \sigma_{1}^{-1}, \ldots, \sigma_{n}^{-1}\right], \\
B_{n}^{\prime}:=T^{-1} A_{n}=K\left[H_{1}, \ldots, H_{n}\right]\left[x_{1}^{ \pm 1}, \ldots, x_{n}^{ \pm 1} ; \sigma_{1}, \ldots, \sigma_{n}\right] .
\end{gathered}
$$

Алгебра Вейля $\left(A_{n},[\cdot, \cdot]\right)$ является $\mathbb{Z}^{n}$-градуированной алгеброй Ли, т. е. $\left[A_{n, \alpha}\right.$, $\left.A_{n, \beta}\right] \subseteq A_{n, \alpha+\beta}$ для всех элементов $\alpha, \beta \in \mathbb{Z}^{n}$. По определению $\mathfrak{u}_{n}$ и $\operatorname{Der}_{K}\left(P_{n}\right)$ являются $\mathbb{Z}^{n}$-градуированными подалгебрами Ли алгебры Ли $A_{n}$.

Алгебра Ли $\mathfrak{u}_{n}$ содержит конечно- и бесконечномерные максимальные абелевы подалгебры Ли, как показывает следующая лемма.

Лемма 2.5. 1) $\mathcal{D}_{n}:=\bigoplus_{i=1}^{n} K_{i}$ является максималъной абелевой подалгеброй Ли алгебры Ли $\mathfrak{u}_{n}$, причем $\operatorname{Cen}_{\mathfrak{u}_{n}}\left(\mathcal{D}_{n}\right)=\mathcal{D}_{n}$.

2) Идеал $P_{n-1} \partial_{n}$ алгебры Ли $\mathfrak{u}_{n}$ является максимальной абелевой подалгеброй Ли алгебры $\mathfrak{u}_{n}$, причем $\operatorname{Cen}_{\mathfrak{u}_{n}}\left(P_{n-1} \partial_{n}\right)=P_{n-1} \partial_{n}$.

ДокАзАтельство. Утверждение 1) вытекает из следующих двух фактов: $\operatorname{Cen}_{A_{n}}\left(\mathcal{D}_{n}\right)=K\left[\partial_{1}, \ldots, \partial_{n}\right]($ см. $(2.7))$ и $\operatorname{Cen}_{\mathfrak{u}_{n}}\left(\mathcal{D}_{n}\right)=\mathfrak{u}_{n} \cap K\left[\partial_{1}, \ldots, \partial_{n}\right]=\mathcal{D}_{n}$.

Докажем утверждение 2$)$. Из равенства $\operatorname{Cen}_{A_{n-1}}\left(P_{n-1}\right)=P_{n-1}$ следует, что

$$
\operatorname{Cen}_{A_{n-1} \otimes K\left[\partial_{n}\right]}\left(P_{n-1} \partial_{n}\right)=\operatorname{Cen}_{A_{n-1}}\left(P_{n-1}\right) \otimes K\left[\partial_{n}\right]=P_{n-1}\left[\partial_{n}\right] .
$$

Тогда $\operatorname{Cen}_{\mathfrak{u}_{n}}\left(P_{n-1} \partial_{n}\right)=\mathfrak{u}_{n} \cap P_{n-1}\left[\partial_{n}\right]=P_{n-1} \partial_{n}$. Значит, идеал $P_{n-1} \partial_{n}$ является максимальной абелевой подалгеброй Ли алгебры $\mathfrak{u}_{n}$. Лемма доказана.

Вложение $\mathfrak{u}_{n} \subseteq A_{n-1} \otimes K\left[\partial_{n}\right]$ индуцирует гомоморфизм алгебр

$$
\chi_{n}: U_{n} \rightarrow A_{n-1} \otimes K\left[\partial_{n}\right], \quad X_{\alpha, i} \mapsto x^{\alpha} \partial_{i} .
$$

Образ и ядро $\chi_{n}$ вычисляются в теореме 2.6. В силу следствия $\left.2.3,2\right)$ гомоморфизм $\chi_{n}$ продолжается до гомоморфизма алгебр

$$
\chi_{n}: S^{-1} U_{n} \rightarrow S^{-1}\left(A_{n-1} \otimes K\left[\partial_{n}\right]\right)=B_{n-1} \otimes K\left[\partial_{n}, \partial_{n}^{-1}\right],
$$

где $S=\left\{\partial^{\alpha} \mid \alpha \in \mathbb{N}^{\alpha}\right\}$. Очевидно, что $\chi_{n}\left(S^{-1} U_{n}\right)=B_{n-1} \otimes K\left[\partial_{n}, \partial_{n}^{-1}\right]$ (так как $X_{\alpha, i} \partial_{i}^{-1} \mapsto x^{\alpha} \partial_{i} \partial_{i}^{-1}=x^{\alpha}$ и $\left.x_{i}=\partial_{i}^{-1} H_{i}\right)$.

Определим отношение $\prec$ на множестве $\mathbb{N}^{n}$ : будем записывать $\alpha \prec \beta$ для элементов $\alpha=\left(\alpha_{i}\right)$ и $\beta=\left(\beta_{i}\right)$ из $\mathbb{N}^{n}$ тогда и только тогда, когда либо $\alpha=0$, а $\beta$ произвольно (т. е. $0 \prec \beta$ для всех $\beta \in \mathbb{N}^{n}$ ), либо $\alpha \neq 0, \beta \neq 0$ и $\max \{i \mid$ $\left.\alpha_{i} \neq 0\right\}<\max \left\{i \mid \beta_{i} \neq 0\right\}$. Ясно, что из $\alpha \prec \beta$ и $\beta \prec \gamma$ следует $\alpha \prec \gamma ; \alpha \prec \alpha$ тогда и только тогда, когда $\alpha=0$; для всех $\alpha, \beta \in \mathbb{N}^{n} \backslash\{0\}$ из $\alpha \prec \beta$ следует $\beta \nprec \alpha$.

Теорема 2.6. 1) Множество $W_{n}:=\left\{x^{\alpha} \partial^{\beta} \mid \alpha, \beta \in \mathbb{N}^{n} ; \alpha \prec \beta\right\}$ является $K$ базисом алгебры $U_{n}^{\prime}:=\chi_{n}\left(U_{n}\right)$.

2) Ядро гомоморфизма алгебр $\chi_{n}$ является идеалом алгебры $U_{n}$, порожденным элементами $X_{\alpha, i} X_{\beta, j}-X_{0, i} X_{\alpha+\beta, j}$, где $i \leqslant j, \alpha \in \mathbb{N}^{i-1} u \beta \in \mathbb{N}^{j-1}$.

3) Гомоморфизм $\chi_{n}$ не является сюргективным отображением.

4) Алгебра $U_{n}^{\prime}$ не является ни конечно порожденной, ни лево-, ни правонётеровой алгеброй. 
ДокАзАтельство. Сначала докажем утверждение 1). Элементы множества $W_{n}$ являются $K$-линейно независимыми элементами, так как они являются таковыми как элементы алгебры $A_{n-1} \otimes K\left[\partial_{n}\right]$. Пусть $\mathcal{I}_{n}:=\sum_{w \in W_{n}} K w$. Необходимо показать, что $\chi_{n}\left(U_{n}\right)=\mathcal{I}_{n}$. Алгебра $\chi_{n}\left(U_{n}\right)$ порождается элементами $\chi_{n}\left(X_{\alpha, i}\right)=x^{\alpha} \partial_{i}$. Используя соотношения

$$
x^{\alpha} \partial_{i} x^{\beta} \partial_{i}=x^{\alpha+\beta} \partial_{i}^{2}, \quad\left[x^{\alpha} \partial_{i}, x^{\gamma} \partial_{j}\right]=\gamma_{i} x^{\alpha+\gamma-e_{i}} \partial_{i} \partial_{j}, \quad i<j,
$$

видим, что алгебра $\chi_{n}\left(U_{n}\right)$ содержится в линейной оболочке, скажем, $\mathcal{I}_{n}^{\prime}$ элементов

$$
\partial_{1}^{\beta_{1}} x^{\alpha_{2}} \partial_{2}^{\beta_{2}} \cdots x^{\alpha_{i}} \partial_{i}^{\beta_{i}} \cdots x^{\alpha_{n}} \partial_{n}^{\beta_{n}}, \quad \beta_{i} \in \mathbb{N}, \quad \alpha_{i} \in \mathbb{N}^{i-1}, \quad i=2, \ldots, n .
$$

Используя соотношения коммутирования $\left[\partial_{i}, x_{j}\right]=\delta_{i j}$ (где $\delta_{i j}$ - дельта-функция Кронекера), каждый такой элемент можно записать в виде линейной комбинации некоторых элементов из $W_{n}$. Значит, $\chi_{n}\left(U_{n}\right) \subseteq \mathcal{I}_{n}^{\prime} \subseteq \mathcal{I}_{n}$.

Чтобы доказать, что выполняется обратное вложение $\chi_{n}\left(U_{n}\right) \supseteq \mathcal{I}_{n}$ и тем самым завершить доказательство утверждения 1), нам нужно показать, что каждый элемент $w \neq 1$ из $W_{n}$ принадлежит алгебре $\chi_{n}\left(U_{n}\right)$. Элемент $w \neq 1$ является произведением

$$
x_{1}^{\alpha_{1}} \cdots x_{s}^{\alpha_{s}} \partial_{1}^{\beta_{1}} \cdots \partial_{t}^{\beta_{t}} \quad \text { для некоторых } \quad \alpha_{i}, \beta_{j} \in \mathbb{N}, \quad s<t, \quad \beta_{t} \neq 0 .
$$

Случай, когда $\alpha_{1}=\cdots=\alpha_{s}=0$, очевиден. Итак, можно считать, что $\alpha_{s} \neq 0$. Каждый элемент $x_{1}^{\alpha_{1}} \cdots x_{s}^{\alpha_{s}} \partial_{1}^{\beta_{1}} \cdots \partial_{s}^{\beta_{s}}$ алгебры Вейля $A_{s}$ можно записать в виде суммы $\sum_{u, v \in \mathbb{N}^{s}} \lambda_{u v} \partial^{u} x^{v}$, где $\lambda_{u v} \in K$. Теперь

$$
w=\left(\sum_{u, v \in \mathbb{N}^{s}} \lambda_{u v} \partial^{u} x^{v}\right) \partial_{s+1}^{\beta_{s+1}} \cdots \partial_{t}^{\beta_{t}}=\sum_{u, v \in \mathbb{N}^{s}} \lambda_{u v} \partial^{u} \partial_{s+1}^{\beta_{s+1}} \cdots \partial_{t-1}^{\beta_{t-1}}\left(x^{v} \partial_{t}^{\beta_{t}}\right) \in \chi_{n}\left(U_{n}\right) .
$$

Доказательство утверждения 1) закончено. Более того, из последнего шага следует, что множество

$$
W_{n}^{\prime}:=\left\{\partial^{\alpha}, \partial^{\beta} x^{\nu} \partial_{t}^{i} \mid \alpha \in \mathbb{N}^{n} ; i \geqslant 1 ; \beta, \nu \in \mathbb{N}^{t-1}, \nu \neq 0 ; t=2, \ldots, n\right\}
$$

также является $K$-базисом алгебры $\chi_{n}\left(U_{n}\right)$. Подробнее, алгебра $\chi_{n}\left(U_{n}\right)$ является линейной оболочкой $W_{n}^{\prime}$, а элементы множества $W_{n}^{\prime}$ линейно независимы, так как они линейно независимы как элементы алгебры Вейля $A_{n}$. Значит, $W_{n}^{\prime}$ является $K$-базисом алгебры $\chi_{n}\left(U_{n}\right)$. Этот базис используется в доказательстве утверждения 2).

Докажем утверждение 2). Пусть $I$ - идеал алгебры $U_{n}$, порожденный элементами из утверждения 2) (кандидатами на роль порождающих $\left.\operatorname{ker}\left(\chi_{n}\right)\right)$. Для каждого элемента $w^{\prime}$ множества $W_{n}^{\prime}$, т. е. для $\partial^{\alpha}$ и $\partial^{\beta} x^{v} \partial_{t}^{i}$, выберем элемент $w^{\prime \prime} \in \chi_{n}^{-1}\left(w^{\prime}\right)$ следующим образом:

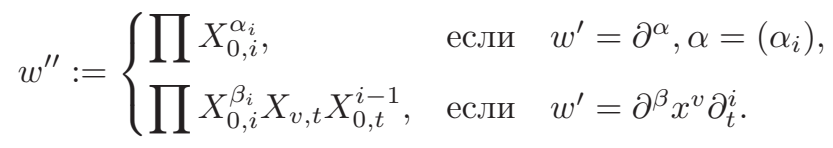

Итак, $\chi_{n}\left(w^{\prime \prime}\right)=w^{\prime}$ для всех элементов $w^{\prime} \in W_{n}^{\prime}$. Пусть $W_{n}^{\prime \prime}-$ множество всех элементов $w^{\prime \prime}$. Элементы из $W_{n}^{\prime \prime}$ линейно независимы в алгебре $U_{n}$ как 
прообразы линейно независимых элементов. Пусть $\mathcal{I}_{n}^{\prime \prime}:=\sum_{w^{\prime \prime} \in W_{n}^{\prime \prime}} K w^{\prime \prime}$. Для завершения доказательства утверждения 2) достаточно показать, что

$$
U_{n}=\mathcal{I}_{n}^{\prime \prime}+I
$$

(Предположим, что выполняется равенство. Поскольку $I \subseteq \operatorname{ker}\left(\chi_{n}\right)$ и множество $W_{n}^{\prime \prime}$ отображается биективно на базис $W_{n}^{\prime}$ алгебры $\chi_{n}\left(U_{n}\right)$, из этих двух фактов с необходимостью следует, что $I=\operatorname{ker}\left(\chi_{n}\right)$.) Чтобы доказать равенство $U_{n}=\mathcal{I}_{n}^{\prime \prime}+I$, следуем схеме доказательства утверждения 1$)$. Из соотношений $(2.2)$ и $X_{\alpha, i} X_{\beta, i} \equiv X_{0, i} X_{\alpha+\beta, i} \bmod I$ следует, что линейная оболочка элементов

$$
\begin{aligned}
\left\{X_{0,1}^{\beta_{1}}\left(X_{0,2}^{\beta_{2}} X_{\alpha_{2}, 2}^{\prime}\right) \cdots\left(X_{0, i}^{\beta_{i}} X_{\alpha_{i}, i}^{\prime}\right) \cdots\left(X_{0, n}^{\beta_{n}} X_{\alpha_{n}, n}^{\prime}\right) \mid \beta_{i} \in \mathbb{N}, \alpha_{i} \in \mathbb{N}^{i-1},\right. \\
\left.X_{\alpha_{i}, i}^{\prime} \in\left\{1, X_{\alpha_{i}, i}\right\}, i=2, \ldots, n\right\},
\end{aligned}
$$

где

$$
\left(X_{0, i}^{\beta_{i}} X_{\alpha_{i}, i}^{\prime}\right)=\left\{\begin{array}{lll}
1, & \text { если } & \beta_{i}=0, \\
X_{0, i}^{\beta_{i}} X_{\alpha_{i}, i}^{\prime}, & \text { если } & \beta_{i} \neq 0,
\end{array}\right.
$$

порождает алгебру $U_{n}$ по модулю идеала $I$. Используя порождающие для идеала $I$ и соотношения (2.2), каждый из этих элементов можно записать в виде линейной комбинации элементов $w^{\prime \prime}$ (определенных выше). Тогда $U_{n}=\mathcal{I}_{n}^{\prime \prime}+I$.

Теперь докажем утверждение 3 ). Предположим, что гомоморфизм $\chi_{n}$ является сюръективным отображением; получим противоречие. Идеал $\mathfrak{a}:=U_{n} \mathfrak{u}_{n} U_{n}$ алгебры $U_{n}$ содержит $\operatorname{ker}\left(\chi_{n}\right), U_{n} / \mathfrak{a} \simeq K, \chi_{n}(\mathfrak{a})$ - идеал алгебры $\chi_{n}\left(U_{n}\right)=$ $A_{n-1} \otimes K\left[\partial_{n}\right]$, и

$A_{n-1} \otimes K\left[\partial_{n}\right] / \chi_{n}(\mathfrak{a})=\chi_{n}\left(U_{n}\right) / \chi_{n}(\mathfrak{a})=\chi_{n}(K+\mathfrak{a}) / \chi_{n}(\mathfrak{a})=\left(K+\chi_{n}(\mathfrak{a})\right) / \chi_{n}(\mathfrak{a}) \simeq K$.

Алгебра Вейля $A_{n-1}$ является простой бесконечномерной алгеброй, поэтому она отображается изоморфно на свой образ под действием гомоморфизма алгебр

$$
A_{n-1} \rightarrow A_{n-1} \otimes K\left[\partial_{n}\right] / \chi_{n}(\mathfrak{a}) \simeq K, \quad a \mapsto a \otimes 1+\chi_{n}(\mathfrak{a}) .
$$

Противоречие.

Утверждение 4) следует из предложения 3.13, где доказано более сильное утверждение.

СлЕДСТвиЕ 2.7. Множество $W_{n}^{\prime}($ см. (2.12)) является K-базисом алгебpъь $U_{n}^{\prime}$.

ДокАЗАТЕльство. Этот факт установлен в доказательстве утверждения 1) теоремы 2.6 .

\section{§ 3. Классификация идеалов алгебры Ли $\mathfrak{u}_{n}$}

В этом параграфе вводятся однорядная и центральная размерности и дается классификация идеалов алгебры Ли $\mathfrak{u}_{n}$ (теорема $\left.3.6,1\right)$ ). Доказывается, что алгебра Ли $\mathfrak{u}_{n}$ является однорядной, артиновой, но не нётеровой алгеброй Ли однорядной размерности $\mathrm{u} \cdot \operatorname{dim}\left(\mathfrak{u}_{n}\right)=\omega^{n-1}+\omega^{n-2}+\cdots+\omega+1($ теорема $\left.3.6,2)\right)$; 
каждый идеал из $\mathfrak{u}_{n}$ является характеристическим идеалом (следствие 3.11 ); найден гиперцентральный ряд алгебры Ли $\mathfrak{u}_{n}$ и показано, что центральной размерностью алгебры Ли $\mathfrak{u}_{n}$ является с. $\operatorname{dim}\left(\mathfrak{u}_{n}\right)=\omega^{n-1}+\omega^{n-2}+\cdots+\omega+1$ (теорема 3.10).

3.1. Однорядная размерность. Пусть $(S, \leqslant)$ - частично упорядоченное множество, т. е. множество $S$ допускает отношение $\leqslant$, удовлетворяющее для всех $a, b, c \in S$ трем условиям:

(i) $a \leqslant a$;

(ii) из $a \leqslant b$ и $b \leqslant a$ следует $a=b$;

(iii) из $a \leqslant b$ и $b \leqslant c$ следует $a \leqslant c$.

Частично упорядоченное множество $(S, \leqslant)$ называется артиновым частично упорядоченным множеством, если каждое непустое подмножество $T$ из $S$ обладает наименъшим элементом, скажем, $t \in T$, т. е. $t \leqslant t^{\prime}$ для всех $t^{\prime} \in T$. Частично упорядоченное множество $(S, \leqslant)$ линейно упорядочено, если для всех элементов $a, b \in S$ либо $a \leqslant b$, либо $b \leqslant a$. Биекция $f: S \rightarrow S^{\prime}$ между двумя частично упорядоченными множествами $(S, \leqslant)$ и $\left(S^{\prime}, \leqslant\right)$ называется изоморфизмом, если из $a \leqslant b$ в $S$ следует $f(a) \leqslant f(b)$ в $S^{\prime}$. Напомним, что ординальные числа - это классы изоморфизмов линейно упорядоченных артиновых множеств (т.е. вполне упорядоченных множеств). Ординальное число (класс изоморфизма) линейно упорядоченного артинова множества $(S, \leqslant)$ обозначается через $\operatorname{ord}(S)$. Класс всех ординальных чисел обозначается через W. Класс W линейно упорядочен по включению $\leqslant$ и артинов. В W определены ассоциативное сложение + и ассоциативное умножение $\cdot$, продолжающие сложение и умножение натуральных чисел. Каждое ненулевое натуральное число $n$ отождествляется с $\operatorname{ord}(1<2<\cdots<n)$. Пусть $\omega:=\operatorname{ord}(\mathbb{N}, \leqslant)$. (Более подробно с ординальными числами можно ознакомиться в книге [5].)

ОПРедЕЛЕНИЕ 3.1. Пусть $(S, \leqslant)$ - частично упорядоченное множество. Однорядной размерностъю u. $\operatorname{dim}(S)$ множества $S$ называется супремум ординальных чисел $\operatorname{ord}(\mathcal{I})$, где $\mathcal{I}$ пробегает все артиновы линейно упорядоченные подмножества из $S$.

Для алгебры Ли $\mathcal{G}$ пусть $\mathcal{J}_{0}(\mathcal{G})$ и $\mathcal{J}(\mathcal{G})$ - множества всех и всех ненулевых идеалов алгебры Ли $\mathcal{G}$ соответственно. Таким образом, $\mathcal{J}_{0}(\mathcal{G})=\mathcal{J}(\mathcal{G}) \cup\{0\}$. Множества $\mathcal{J}_{0}(\mathcal{G})$ и $\mathcal{J}(\mathcal{G})$ являются частично упорядоченными множествами по включению. Алгебра Ли $\mathcal{G}$ называется артиновой (соответственно, нётеровой), если частично упорядоченное множество $\mathcal{J}(\mathcal{G})$ артиново (соответственно, нётерово). Это означает, что каждая убывающая (соответственно, возрастающая) цепочка идеалов стабилизируется. Алгебра Ли $\mathcal{G}$ называется однорядной алгеброй Ли, если частично упорядоченное множество $\mathcal{J}(\mathcal{G})$ является линейно упорядоченным множеством. Это означает, что для любых двух идеалов $\mathfrak{a}$ и $\mathfrak{b}$ алгебры Ли $\mathcal{G}$ либо $\mathfrak{a} \subseteq \mathfrak{b}$, либо $\mathfrak{b} \subseteq \mathfrak{a}$.

ОПРЕДЕлЕниЕ 3.2. Пусть $\mathcal{G}$ - артинова однорядная алгебра Ли. Ординальное число $\mathrm{u} \operatorname{dim}(\mathcal{G}):=\operatorname{ord}(\mathcal{J}(\mathcal{G}))$ артинова линейно упорядоченного множества $\mathcal{J}(\mathcal{G})$ ненулевых идеалов алгебры $\mathcal{G}$ называется однорядной размерностью алгебры Ли $\mathcal{G}$. Для произвольной алгебры Ли $\mathcal{G}$ однорядная размерность 
u. $\operatorname{dim}(\mathcal{G})$ - это супремум ординальных чисел $\operatorname{ord}(\mathcal{I})$, где $\mathcal{I}$ пробегает все артиновы линейно упорядоченные множества идеалов.

Если $\mathcal{G}$ - нётерова алгебра Ли, то и. $\operatorname{dim}(\mathcal{G}) \leqslant \omega$. Таким образом, однорядная размерность является мерой отклонения от условия нётеровости. Понятие однорядной размерности имеет смысл для любого класса алгебр (ассоциативных, йордановых и т. п.).

Пусть $A$ - алгебра, а $M$ - ее модуль, и пусть $\mathcal{J}_{l}(A)$ и $\mathcal{M}(M)$ - множества всех ненулевых левых идеалов алгебры $A$ и всех ненулевых подмодулей из $M$ соответственно. Они являются частично упорядоченными множествами относительно $\subseteq$. Левая однорядная размерность алгебры $A$ определяется как u. $\operatorname{dim}(A):=\mathrm{u} \cdot \operatorname{dim}\left(\mathcal{J}_{l}(A)\right)$ и однорядная размерность $A$-модуля $M$ определяется как и. $\operatorname{dim}(M):=$ u. $\operatorname{dim}(\mathcal{M}(M))$.

3.2. Артиново линейное упорядочение на каноническом базисе $\mathcal{B}_{n}$ алгебры $\mathfrak{u}_{n}$. Определим артиново линейное упорядочение $\leqslant$ на каноническом базисе $\mathcal{B}_{n}$ для алгебры Ли $\mathfrak{u}_{n}$ по правилу $X_{\alpha, i}>X_{\beta, j}$ тогда и только тогда, когда $i<j$ или $i=j$ и $\alpha_{n-1}=\beta_{n-1}, \ldots, \alpha_{m+1}=\beta_{m+1}, \alpha_{m}>\beta_{m}$ для некоторого $m$.

ПримеР 3.3. Для $n=2$ имеем $\partial_{2}<x_{1} \partial_{2}<x_{1}^{2} \partial_{2}<\cdots<\partial_{1}$. Для $n=3$ имеем

$$
\begin{gathered}
\partial_{3}<x_{1} \partial_{3}<x_{1}^{2} \partial_{3}<\cdots<x_{2} \partial_{3}<x_{1} x_{2} \partial_{3}<x_{1}^{2} x_{2} \partial_{3}<\cdots \\
\cdots<x_{2}^{i} \partial_{3}<x_{1} x_{2}^{i} \partial_{3}<x_{1}^{2} x_{2}^{i} \partial_{3}<\cdots<\partial_{2}<x_{1} \partial_{2}<x_{1}^{2} \partial_{2}<\cdots<\partial_{1}
\end{gathered}
$$

Следующая лемма является простым следствием определения упорядочения $<$; записываем $0<X_{\alpha, i}$ для всех $X_{\alpha, i} \in \mathcal{B}_{n}$.

Лемма 3.4. Если $X_{\alpha, i}>X_{\beta, j}$, mo:

1) $X_{\alpha+\gamma, i}>X_{\beta+\gamma, j}$ для всех $\gamma \in \mathbb{N}^{i-1}$;

2) $X_{\alpha-\gamma, i}>X_{\beta-\gamma, j}$ для всех $\gamma \in \mathbb{N}^{i-1} \operatorname{ma\kappa ux,~что~} \alpha-\gamma \in \mathbb{N}^{i-1} u \beta-\gamma \in \mathbb{N}^{j-1}$;

3) $\left[\partial_{k}, X_{\alpha, i}\right]>\left[\partial_{k}, X_{\beta, j}\right]$ для всех $k=1, \ldots, i-1$ maких, что $\alpha_{k} \neq 0$;

4) $\left[X_{\gamma, k}, X_{\alpha, i}\right]>\left[X_{\gamma, k}, X_{\beta, j}\right]$ для всех $X_{\gamma, k}>X_{\alpha, i} \operatorname{ma\kappa ux,~что~}\left[X_{\gamma, k}, X_{\alpha, i}\right] \neq 0$, m.e. $\alpha_{k} \neq 0$.

Пусть $\Omega_{n}$ - множество индексов $\{(\alpha, i)\}$, параметризующих канонический базис $\left\{X_{\alpha, i}\right\}$ алгебры Ли $\mathfrak{u}_{n}$. Множество $\left(\Omega_{n}, \leqslant\right)$ является артиновым линейно упорядоченным множеством, где $(\alpha, i) \geqslant(\beta, j)$ тогда и только тогда, когда $X_{\alpha, i} \geqslant X_{\beta, j}$, которое изоморфно артинову линейно упорядоченному множеству $\left(\mathcal{B}_{n}, \leqslant\right)$ посредством $(\alpha, i) \mapsto X_{\alpha, i}$. Отождествим частично упорядоченные множества $\left(\Omega_{n}, \leqslant\right)$ и $\left(\mathcal{B}_{n}, \leqslant\right)$ с помощью этого изоморфизма. Очевидно, что

$$
\operatorname{ord}\left(\mathcal{B}_{n}\right)=\operatorname{ord}\left(\Omega_{n}\right)=\omega^{n-1}+\omega^{n-2}+\cdots+\omega+1
$$

$\Omega_{2} \subset \Omega_{3} \subset \cdots$ и $\mathcal{B}_{2} \subset \mathcal{B}_{3} \subset \cdots$. Пусть $\left[1, \operatorname{ord}\left(\Omega_{n}\right)\right]:=\left\{\lambda \in \mathrm{W} \mid 1 \leqslant \lambda \leqslant \operatorname{ord}\left(\Omega_{n}\right)\right\}$. В силу $(2.2)$ если $\left[X_{\alpha, i}, X_{\beta, j}\right] \neq 0$, то

$$
\left[X_{\alpha, i}, X_{\beta, j}\right]<\min \left\{X_{\alpha, i}, X_{\beta, j}\right\} .
$$


В силу (3.2) отображение

$$
\rho_{n}:\left[1, \operatorname{ord}\left(\Omega_{n}\right)\right] \rightarrow \mathcal{J}\left(\mathfrak{u}_{n}\right), \quad \lambda \mapsto I_{\lambda}:=I_{\lambda, n}:=\bigoplus_{(\alpha, i) \leqslant \lambda} K X_{\alpha, i},
$$

является мономорфизмом частично упорядоченных множеств $\left(\rho_{n}-\right.$ сохраняющая порядок инъекция). Мы докажем, что отображение $\rho_{n}$ является биекцией $($ теорема $3.6,1))$ и в результате получим классификацию всех идеалов алгебры Ли $\mathfrak{u}_{n}$. Каждый ненулевой элемент $u$ из $\mathfrak{u}_{n}$ является конечной линейной комбинацией:

$$
u=\lambda X_{\alpha, i}+\mu X_{\beta, j}+\cdots+\nu X_{\sigma, k}=\lambda X_{\alpha, i}+\cdots,
$$

где $\lambda, \mu, \ldots, \nu \in K^{*}$ и $X_{\alpha, i}>X_{\beta, j}>\cdots>X_{\sigma, k}$. Элементы $\lambda X_{\alpha, i}$ и $\lambda \in K^{*}$ называются старшим членом и старшим коэффиииентом элемента и соответственно, а ординальное число, обозначаемое через $\operatorname{ord}\left(X_{\alpha, i}\right)=\operatorname{ord}(\alpha, i) \in\left[1, \operatorname{ord}\left(\Omega_{n}\right)\right]$, по определению равное ординальному числу, представляющему артиново линейно упорядоченное множество $\left\{(\beta, j) \in \Omega_{n} \mid(\beta, j) \leqslant(\alpha, i)\right\}$, называется ординальной степенъю элемента $u$, обозначаемой через $\operatorname{ord}(u)$ (надеемся, что эти обозначения не вызовут путаницы). Для всех ненулевых элементов $u, v \in \mathfrak{u}_{n}$ и $\lambda \in K^{*}$ выполнено:

(i) $\operatorname{ord}(u+v) \leqslant \max \{\operatorname{ord}(u), \operatorname{ord}(v)\}$, как только $u+v \neq 0$;

(ii) $\operatorname{ord}(\lambda u)=\operatorname{ord}(u)$;

(iii) $\operatorname{ord}([u, v])<\min \{\operatorname{ord}(u), \operatorname{ord}(v)\}$, как только $[u, v] \neq 0$;

(iv) $\operatorname{ord}(\sigma(u))=\operatorname{ord}(u)$ для всех автоморфизмов $\sigma$ алгебры Ли $\mathfrak{u}_{n}$ (следствие 3.12$)$.

3.3. Классификация идеалов алгебры Ли $\mathfrak{u}_{n}$. Следующая лемма является решающим фактом в доказательстве теоремы 3.6.

Лемма 3.5. Предположим, что I - ненулевой идеал алгебры Ли $\mathfrak{u}_{n}$. Тогда $I=\bigcup_{0 \neq u \in I} I_{\operatorname{ord}(u)}$. В частности, $(v)=I_{\operatorname{ord}(v)}$ для всех ненулевых элементов $v$ из $\mathfrak{u}_{n}$, где $(v)$ - идеал алгебры Ли $\mathfrak{u}_{n}$, порожденный элементом $v$.

ДокАзАтельство. Достаточно показать, что выполнено второе утверждение, т. е. $(v)=I_{\mathrm{ord}(v)}$, так как тогда

$$
I=\sum_{0 \neq u \in I}(u)=\sum_{0 \neq u \in I} I_{\operatorname{ord}(u)}=\bigcup_{0 \neq u \in I} I_{\operatorname{ord}(u)} .
$$

Чтобы доказать равенство $(v)=I_{\text {ord }(v)}$, используем двойную индукцию: сначала по $n \geqslant 2$, а затем для фиксированного $n$ вторую индукцию по $\lambda=\operatorname{ord}(v)$. Без ограничения общности можно считать, что старший коэффициент элемента $v$ равен 1. Заметим, что $\operatorname{ord}(v)$ - непредельный ординал.

А. Пусть $n=2$. Если $\lambda=1$, то $v=\partial_{2}$ и, значит, $(v)=K \partial_{2}=I_{1}$, как и требуется. Предположим, что $\lambda>1$ и утверждение верно для всех непредельных ординалов $\lambda^{\prime}$ таких, что $\lambda^{\prime}<\lambda$.

Случай 1: $\lambda=\operatorname{ord}\left(x_{1}^{i} \partial_{2}\right)$ для некоторого $i \geqslant 1$, т. е.

$$
v=x_{1}^{i} \partial_{2}+\mu_{i-1} x_{1}^{i-1} \partial_{2}+\cdots+\mu_{0} \partial_{2}
$$


для некоторых скаляров $\mu_{j} \in K$. Пусть $\delta=\operatorname{ad}\left(\partial_{1}\right)$. Тогда $I_{\lambda} \supseteq(v) \supseteq$ $\sum_{j=0}^{i} K \delta^{j}(v)=I_{\lambda}$, т. е. $(v)=I_{\lambda}$.

Случай 2: $\lambda=\operatorname{ord}\left(\partial_{1}\right)=\omega+1$, т. е. $v=\partial_{1}+p \partial_{2}$ для некоторого элемента $p \in P_{1}$. Для всех $i \geqslant 1$ имеем $(v) \ni\left[v, x_{1}^{i} \partial_{2}\right]=i x_{1}^{i-1} \partial_{2}$. В силу случая 1 имеем $\mathfrak{u}_{2,1} \subseteq(v)$, и потому $\partial_{1} \in(v)$. Значит, $(v)=\mathfrak{u}_{2}=I_{\omega+1}=I_{\operatorname{ord}(v)}$.

В. Предположим, что $n>2$ и утверждение выполняется для всех $n^{\prime}$ таких, что $n^{\prime}<n$. Если $\lambda=1$, то $v \in K^{*} \partial_{n}$, и потому $(v)=K \partial_{n}=I_{1}$, как и требуется. Предположим, что $\lambda>1$ и утверждение верно для всех непредельных ординалов $\lambda^{\prime}$ таких, что $\lambda^{\prime}<\lambda$.

Случай 1: $\lambda=\operatorname{ord}\left(x^{\alpha} \partial_{n}\right)$ для некоторого $0 \neq \alpha \in \mathbb{N}^{n-1}$, т. е. $v=x^{\alpha} \partial_{n}+\cdots$, где три точки означают младшие члены. Следующее утверждение вытекает из $(2.2)$ и определения линейного упорядочения на каноническом базисе $\mathcal{B}_{n}$ алгебры $\mathfrak{u}_{n}$.

Известно следующее утверждение: для каждого непредельного ординала $\lambda^{\prime}$ такого, что $\lambda^{\prime}<\lambda$, существуют элементь из $\mathcal{B}_{n}$, скажем, $a_{1}, \ldots, a_{s}$ тиna $X_{\beta, i}, i \neq n$, maкue, чmo

$$
\operatorname{ord}\left(\operatorname{ad}\left(a_{1}\right) \cdots \operatorname{ad}\left(a_{s}\right)(v)\right)=\lambda^{\prime} .
$$

Из этого утверждения индукцией по $\lambda$ следует, что $(v)=I_{\operatorname{ord}(v)}$.

Случай 2: $v=\partial_{i}+\cdots$ для некоторого $i$ такого, что $i<n$. Для всех элементов $x^{\beta} \partial_{i+1}$ при $\beta \in \mathbb{N}^{i}$ и $\beta_{i} \neq 0$ имеем

$$
(v) \ni\left[v, x^{\beta} \partial_{i+1}\right]=\beta_{i} x^{\beta-e_{i}} \partial_{i+1}+\cdots,
$$

и потому $\operatorname{ord}\left(\left[v, x^{\beta} \partial_{i+1}\right]\right)=\operatorname{ord}\left(x^{\beta-e_{i}} \partial_{i+1}\right)$. По индукции $(v) \supseteq \bigcup_{\mu<\lambda} I_{\mu}=: J$. Однако $I_{\lambda} \supseteq(v)=K v+J=K \partial_{i}+J=I_{\lambda}$, и потому $(v)=I_{\lambda}$.

Случай 3: $v=x^{\alpha} \partial_{i}+\cdots$ для некоторого $i$ такого, что $i<n$ и $\alpha \in \mathbb{N}^{i-1} \backslash\{0\}$. Заметим, что

$$
(v) \ni \prod_{j=1}^{i-1} \frac{\operatorname{ad}\left(\partial_{j}\right)^{\alpha_{j}}}{\alpha_{j} !}(v)=\partial_{i}+\cdots .
$$

В силу случая 2 имеем $I_{\text {ord }\left(\partial_{i}\right)} \subseteq(v)$. Поскольку $\mathfrak{u}_{n, i+1} \subseteq I_{\operatorname{ord}\left(\partial_{i}\right)}$, имеем $\mathfrak{u}_{n, i+1} \subseteq$ $(v)$. В силу $(2.6)$ получаем $\mathfrak{u}_{i} \simeq \mathfrak{u}_{n} / \mathfrak{u}_{n, i+1}$. По предположению имеем $i<n$. Рассматривая элемент $v+\mathfrak{u}_{n, i+1} \in \mathfrak{u}_{i}$, доказываем лемму в случае 3 индукцией по $i$. Лемма доказана.

Пусть $u, v \in \mathfrak{u}_{n} \backslash\{0\}$. Тогда $(u) \subseteq(v)$, если и только если $\operatorname{ord}(u) \leqslant \operatorname{ord}(v)$.

ТЕОРема 3.6. 1) Отображение (3.3) является биекиией.

2) Алгебра Ли й является однорядной, артиновой, но не нётеровой алгеброй Ли, и ее однорядная размерность есть и. $\operatorname{dim}\left(\mathfrak{u}_{n}\right)=\operatorname{ord}\left(\Omega_{n}\right)=\omega^{n-1}+$ $\omega^{n-2}+\cdots+\omega+1$.

ДокАзАтельство. Утверждение 1) немедленно следует из леммы 3.5. Утверждение 2) следует из утверждения 1). Теорема доказана.

Идеал $\mathfrak{a}$ алгебры Ли $\mathcal{G}$ называется собственным (соответственно, коконечным $)$, если $\mathfrak{a} \neq 0, \mathcal{G}$ (соответственно, $\left.\operatorname{dim}_{K}(\mathcal{G} / \mathfrak{a})<\infty\right)$. 
СлЕДСТвИЕ 3.7. 1) Идеал $\mathfrak{u}_{n, 2}$ является наибольшим собственным идеалом алгебры Ли $\mathfrak{u}_{n}$.

2) Идеал $\mathfrak{u}_{n, 2}$ является единственным собственным коконечным идеалом алгебры Ли $\mathfrak{u}_{n}$, причем $\operatorname{dim}_{K}\left(\mathfrak{u}_{n} / \mathfrak{u}_{n, 2}\right)=1$.

3) Центр $Z\left(\mathfrak{u}_{n}\right)=K \partial_{n}$ алгебры Ли $\mathfrak{u}_{n}$ является наименъшим ненулевым идеалом алгебрь Ли $\mathfrak{u}_{n}$.

4) Идеалы $I_{s}:=\sum_{i=0}^{s-1} K x_{1}^{i} \partial_{n}, s=1,2, \ldots$, и только они являются конечномерными идеалами алгебры Ли $\mathfrak{u}_{n}$, причем $\operatorname{dim}_{K}\left(I_{s}\right)=s$.

ДокАзАтЕльство. Все утверждения - это несложные следствия теоремы 3.6.

3.4. Централизаторы идеалов алгебры $\mathfrak{u}_{n}$. В сочетании с теоремой 3.6 следующее предложение описывает централизаторы всех идеалов алгебры Ли $\mathfrak{u}_{n}$. Заметим, что централизатор идеала алгебры Ли также является идеалом.

ПРЕДЛОЖЕНИЕ 3.8. 1) Имеем

$$
\operatorname{Cen}_{\mathfrak{u}_{n}}\left(I_{\lambda, n}\right)=\left\{\begin{array}{lll}
K \partial_{n}, & \text { если } & \lambda=\operatorname{ord}\left(\Omega_{n}\right)=\omega^{n-1}+\cdots+\omega+1, \\
I_{\omega^{i}, n}=P_{i} \partial_{n}, & \text { если } & \lambda \in\left(\omega^{n-1}+\cdots+\omega^{i+1}, \omega^{n-1}+\cdots+\omega^{i}\right], \\
& & i=1, \ldots, n-2, \\
\bigoplus_{i=m+1}^{n} P_{i-1} \partial_{i}, & \text { если } & \lambda \in\left(\omega^{m-1}, \omega^{m}\right], m=1, \ldots, n-1, \\
\mathfrak{u}_{n}, & \text { если } & \lambda=1 .
\end{array}\right.
$$

2) Множество $\mathcal{C}\left(\mathfrak{u}_{n}\right)$ всех централизаторов идеалов алгебры Ли $\mathfrak{u}_{n}$ содержит в точности $2 n-1$ элементов, а отображение $\mathcal{C}\left(\mathfrak{u}_{n}\right) \rightarrow \mathcal{C}\left(\mathfrak{u}_{n}\right), C \mapsto$ $\mathrm{Cen}_{\mathfrak{u}_{n}}(C)$, является биекцией. Более того, оно является обращающей вложение инволюиией, m.е. $\operatorname{Cen}_{\mathfrak{u}_{n}}\left(\operatorname{Cen}_{\mathfrak{u}_{n}}(C)\right)=C$ для всех $C \in \mathcal{C}\left(\mathfrak{u}_{n}\right)$.

3) Имеем $\operatorname{Cen}_{\mathfrak{u}_{n}}\left(\mathfrak{u}_{n}\right)=K \partial_{n}, \operatorname{Cen}_{\mathfrak{u}_{n}}\left(K \partial_{n}\right)=\mathfrak{u}_{n}, \operatorname{Cen}_{\mathfrak{u}_{n}}\left(P_{n-1} \partial_{n}\right)=P_{n-1} \partial_{n}$, $\operatorname{Cen}_{\mathfrak{u}_{n}}\left(P_{i} \partial_{n}\right)=\bigoplus_{j=i+1}^{n} P_{j-1} \partial_{j} u \operatorname{Cen}_{\mathfrak{u}_{n}}\left(\bigoplus_{j=i+1}^{n} P_{j-1} \partial_{j}\right)=P_{i} \partial_{n} \partial л я i=1, \ldots, n-2$.

ДокАЗАТЕЛЬСтво. Пусть $C_{\lambda}:=\operatorname{Cen}_{\mathfrak{u}_{n}}\left(I_{\lambda, n}\right)$. Если $\lambda=\operatorname{ord}\left(\Omega_{n}\right)$, то $I_{\lambda, n}=\mathfrak{u}_{n}$ и $C_{\lambda}=Z\left(\mathfrak{u}_{n}\right)=K \partial_{n}$ по предложению $\left.2.1,6\right)$. Если $\lambda=1$, то $I_{1, n}=K \partial_{n}=$ $Z\left(\mathfrak{u}_{n}\right)$, и потому $C_{1}=\mathfrak{u}_{n}$. Можно считать, что $\lambda \neq 1, \operatorname{ord}\left(\Omega_{n}\right)$. Чтобы доказать утверждение 1 ), воспользуемся индукцией по $n \geqslant 2$. При $n=2$ необходимо рассмотреть только случай, когда $\lambda \in(1, \omega]$. В этом случае

$$
I_{\lambda, 2}=\left\{\begin{array}{lll}
\bigoplus_{i=0}^{\lambda-1} x_{1}^{i} \partial_{2}, & \text { если } & 1<\lambda<\omega, \\
P_{1} \partial_{2}, & \text { если } \quad \lambda=\omega,
\end{array}\right.
$$

и потому $C_{\lambda}=P_{1} \partial_{2}$.

Пусть $n>2$; предположим, что утверждение 1) выполнено для всех $n^{\prime}<n$.

Далее мы докажем следующее утверждение: имеем $C_{\omega^{m}}=\bigoplus_{i=m+1}^{n} P_{i-1} \partial_{i}$ для $m=1, \ldots, n-1$. 
Для $m=n-1$ имеем $I_{\omega^{n-1}, n}=P_{n-1} \partial_{n}$ и данное утверждение - это лемма $2.5,2)$. Предположим, что $m<n-1$. Тогда $I_{\omega^{m}, n}=P_{m} \partial_{n} \subset I_{\omega^{n-1}, n}$; отсюда $C_{\omega^{m}} \supseteq C_{\omega^{n-1}}=P_{n-1} \partial_{n}$. Это означает, что $\mathfrak{u}_{n}$-модуль $I_{\omega^{m}, n}$ является также $\left(\mathfrak{u}_{n} / P_{n-1} \partial_{n}\right)$-модулем и $\mathfrak{u}_{n-1}$-модулем, так как $\mathfrak{u}_{n-1} \simeq \mathfrak{u}_{n} / P_{n-1} \partial_{n}$. Отображение

$$
I_{\omega^{m}, n}=P_{m} \partial_{n} \rightarrow I_{\omega^{m}, n-1}=P_{m} \partial_{n-1}, \quad p \partial_{n} \mapsto p \partial_{n-1},
$$

является изоморфизмом $\mathfrak{u}_{n-1}$-модулей (так как $\partial_{n} \in Z\left(\mathfrak{u}_{n}\right)$ и $\partial_{n-1} \in Z\left(\mathfrak{u}_{n-1}\right)$ ), и данное утверждение следует по индукции по $n$.

Предположим, что $1<\lambda \leqslant \omega^{n-1}$, т. е. $\lambda \in\left(\omega^{m-1}, \omega^{m}\right]$ для некоторого $m \in$ $\{1, \ldots, n-1\}$. Надо показать, что $C_{\lambda}=C_{\omega m}$ (см. утверждение). В силу данного утверждения из вложений $I_{\omega^{m-1}, n} \subset I_{\lambda, n} \subseteq I_{\omega^{m}, n}$ следуют вложения $C_{\omega^{m-1}} \supseteq$ $C_{\lambda} \supseteq C_{\omega^{m}}$. Заметим, что $C_{\omega^{m-1}}=C_{\omega^{m}} \oplus P_{m-1} \partial_{m}, x_{m} \partial_{n} \in I_{\lambda, m}$ (так как $\lambda \in$ $\left.\left(\omega^{m-1}, \omega^{m}\right]\right)$ и $\left[p \partial_{m}, x_{m} \partial_{n}\right]=p \partial_{n} \neq 0$ для всех ненулевых элементов $p \in P_{m-1}$. Отсюда следует, что $C_{\lambda}=C_{\omega m}$, как и требуется.

Пусть $\omega^{n-1}<\lambda<\operatorname{ord}\left(\Omega_{n}\right)=\omega^{n-1}+\cdots+\omega+1$, т. е. $\lambda \in\left(\omega^{n-1}+\cdots+\omega^{i+1}\right.$, $\left.\omega^{n-1}+\cdots+\omega^{i}\right]$ для некоторого $i \in\{1, \ldots, n-2\}$, и пусть $\lambda_{i}:=\omega^{n-1}+\cdots+\omega^{i}$. Тогда $I_{\lambda, n} \subseteq I_{\lambda_{i}, n}=\bigoplus_{j=i+1}^{n} P_{j-1} \partial_{j}=\operatorname{Cen}_{\mathfrak{u}_{n}}\left(I_{\omega^{i}, n}\right)$ в силу приведенного утверждения. Значит, $C_{\lambda} \supseteq I_{\omega^{i}, n}=P_{i} \partial_{n}$. Из вложения $I_{\lambda, n} \supset P_{n-1} \partial_{n}=I_{\omega^{n-1}, n}$ следует вложение $C_{\lambda} \subseteq C_{\omega^{n-1}}=P_{n-1} \partial_{n}$ в силу данного утверждения. Тогда из вложения $\left\{\partial_{i+1}, \ldots, \partial_{n}\right\} \subseteq I_{\lambda, n}$ следует, что $C_{\lambda} \subseteq \operatorname{Cen}_{P_{n-1}}\left(\partial_{i+1}, \ldots, \partial_{n}\right) \partial_{n}=$ $P_{i} \partial_{n}=I_{\omega^{i}, n}$. Это означает, что $C_{\lambda}=I_{\omega^{i}, n}$.

Утверждение 2) следует из утверждения 3). Утверждение 3) следует из утверждения 1). Предложение доказано.

3.5. Гиперцентральный ряд и центральная размерность. Для алгебры Ли $\mathcal{G}$ над полем $K$ определим по индукции ее гиперцентралъный ряд $\left\{Z^{(\lambda)}(\mathcal{G})\right\}_{\lambda \in \mathrm{W}}$. Положим $Z^{(0)}(\mathcal{G}):=Z(\mathcal{G})$. Если $\lambda$ - непредельный ординал, т. е. $\lambda=\mu+1$ для некоторого $\mu \in \mathrm{W}$, то

$$
Z^{(\lambda)}(\mathcal{G}):=\pi_{\mu}^{-1}\left(Z\left(\mathcal{G} / Z^{(\mu)}(\mathcal{G})\right)\right), \quad \text { где } \quad \pi_{\mu}: \mathcal{G} \rightarrow \mathcal{G} / Z^{(\mu)}(\mathcal{G}), \quad a \mapsto a+Z^{(\mu)}(\mathcal{G}) .
$$

Если $\lambda$ - предельный ординал, то $Z^{(\lambda)}(\mathcal{G}):=\bigcup_{\mu<\lambda} Z^{(\mu)}(\mathcal{G})$. Если $\lambda \leqslant \mu$, то $Z^{(\lambda)}(\mathcal{G}) \subseteq Z^{(\mu)}(\mathcal{G})$. Итак, $\left\{Z^{(\lambda)}(\mathcal{G})\right\}_{\lambda \in \mathrm{W}}$ - возрастающий класс идеалов алгебры Ли $\mathcal{G}$. Пусть $Z^{(\mathrm{W})}(\mathcal{G}):=\bigcup_{\lambda \in \mathrm{W}} Z^{(\lambda)}(\mathcal{G})$.

ОПРЕДЕЛЕНИЕ 3.9. Минимальное ординальное число $\lambda$ (если оно существует) такое, что $Z^{(\lambda)}(\mathcal{G})=Z^{(\mathrm{W})}(\mathcal{G})$, называется центральной размерностъю алгебры Ли $\mathcal{G}$ и обозначается через с. $\operatorname{dim}(\mathcal{G})$. В том случае, когда такого ординала $\lambda$ не существует, пишем с. $\operatorname{dim}(\mathcal{G})=\mathrm{W}$. Понятие центральной размерности имеет смысл для любого класса алгебр (ассоциативных, йордановых и т. п.).

Алгебра Ли $\mathcal{G}$ центральна, т. е. $Z(\mathcal{G})=0$, тогда и только тогда, когда c. $\operatorname{dim}(\mathcal{G})=0$. Таким образом, центральная размерность измеряет отклонение от “центральности". Следующая теорема описывает гиперцентральный ряд для алгебры Ли $\mathfrak{u}_{n}$ и дает с. $\operatorname{dim}\left(\mathfrak{u}_{n}\right)$.

Теорема 3.10. Гиперцентралъный ряд $\left\{Z^{(\lambda)}\left(\mathfrak{u}_{n}\right)\right\}_{\lambda \in \mathrm{W}}$ стабилизируется 8 точности на шаге $\operatorname{ord}\left(\Omega_{n}\right)=\omega^{n-1}+\omega^{n-2}+\cdots+\omega+1$, m. e. c. $\operatorname{dim}\left(\mathfrak{u}_{n}\right)=$ 
$\operatorname{ord}\left(\Omega_{n}\right)$. Более того, $Z^{(\lambda)}\left(\mathfrak{u}_{n}\right)=I_{\lambda}$ для каждого $\lambda \in\left[1, \operatorname{ord}\left(\Omega_{n}\right)\right]$. В частности, $Z^{\left(\operatorname{codim}\left(\mathfrak{u}_{n}\right)\right)}\left(\mathfrak{u}_{n}\right)=\mathfrak{u}_{n}$.

ДокАзАтельство. Достаточно показать, что $Z^{(\lambda)}=I_{\lambda}$ для всех $\lambda \in$ $\left[1, \operatorname{ord}\left(\Omega_{n}\right)\right]$, где $Z^{(\lambda)}:=Z^{(\lambda)}\left(\mathfrak{u}_{n}\right)$. Воспользуемся индукцией по $\lambda$. Случай $\lambda=1$ следует из предложения 2.1,6): $Z^{(1)}=Z(\mathcal{G})=K \partial_{n}=I_{1}$. Предположим, что $\lambda>1$ и равенство выполняется для всех ординалов $\lambda^{\prime}<\lambda$.

Если $\lambda-$ предельный ординал, то

$$
Z^{(\lambda)}=\bigcup_{\mu<\lambda} Z^{(\mu)}=\bigcup_{\mu<\lambda} I_{\mu}=I_{\lambda} .
$$

Предположим, что $\lambda$ - непредельный ординал, т. е. $\lambda=\mu+1$ для некоторого ординального числа $\mu$.

Случай 1: $\lambda \leqslant \omega^{n-1}$. Тогда с необходимостью имеем $\lambda<\omega^{n-1}$, так как $\lambda$ - непредельный ординал, но $\omega^{n-1}$ предельный. Ясно, что $I_{\lambda} \subseteq Z^{(\lambda)}$ в силу (2.2) (так как $Z^{(\mu)}=I_{\mu}$ по индукции). Предположим, что $I_{\lambda} \neq Z^{(\lambda)}$; тогда с необходимостью $I_{\lambda+1} \subseteq Z^{(\lambda)}$ (по теореме $\left.3.6,1\right)$ ). Если $\lambda=X_{\alpha, i}$ для некоторого $\alpha \in \mathbb{N}^{n-1}$ (напомним, что мы отождествили $\left(\Omega_{n}, \leqslant\right)$ и $\left(\mathcal{B}_{n}, \leqslant\right)$ ), то $\lambda+1=X_{\alpha+e_{1}, n}$ и $X_{\alpha+e_{1}, n} \in I_{\lambda+1}$, но $\left[\partial_{1}, X_{\alpha+e_{1}, n}\right]=\left(\alpha_{1}+1\right) X_{\alpha, n} \notin Z^{(\mu)}$. Противоречие. Значит, $Z^{(\lambda)}=I_{\lambda}$.

Случай 2: $\lambda>\omega^{n-1}$. Заметим, что $Z^{(\lambda)} \supseteq I_{\lambda} \supseteq I_{\omega^{n-1}}=\mathfrak{u}_{n, n}$ и $\mathfrak{u}_{n} / \mathfrak{u}_{n, n} \simeq \mathfrak{u}_{n-1}$ в силу (2.6). Теперь завершим рассуждение индукцией по $n$. База индукции $n=2$ следует из случаев 1,2 для $n=2$. Теорема доказана.

Идеал $I$ алгебры Ли $\mathcal{G}$ называется характеристическим идеалом, если он инвариантен под действием всех автоморфизмов алгебры Ли $\mathcal{G}$, т. е. $\sigma(I)=I$ для всех $\sigma \in \operatorname{Aut}_{K}(\mathcal{G})$. Очевидно, что идеал $I$ характеристический тогда и только тогда, когда $\sigma(I) \subseteq I$ для всех $\sigma \in \operatorname{Aut}_{K}(\mathcal{G})$.

СЛЕДСтвиЕ 3.11. Все идеалы алгебры Ли $\mathfrak{u}_{n}$ являются характеристическими идеалами.

ДокАЗАТЕльство. В силу определения все идеалы в центральном ряду $\left\{Z^{(\lambda)}\left(\mathfrak{u}_{n}\right)\right\}$ являются характеристическими идеалами алгебры $\mathfrak{u}_{n}$, но это все идеалы алгебры Ли $\mathfrak{u}_{n}$ по теореме 3.10. Следствие можно также вывести из теоремы $3.6,2)$.

СЛЕДСтвиЕ 3.12. Для всех ненулевых элементов $u \in \mathfrak{u}_{n} u$ всех автоморфизмов $\sigma$ алгебры Ли $\mathfrak{u}_{n}$ выполняется $\operatorname{ord}(\sigma(u))=\operatorname{ord}(u)$.

ДокАЗАтЕЛЬСтво. Заметим, что

$$
\operatorname{ord}(u)=\min \left\{\lambda \in\left[1, \operatorname{ord}\left(\Omega_{n}\right)\right] \mid u \in I_{\lambda}\right\} .
$$

Теперь утверждение следует из того, что все идеалы $I_{\lambda}$ являются характеристическими (следствие 3.11).

3.6. Подалгебра $U_{n}^{\prime}$ алгебры Вейля $A_{n}$. Пусть $\mathfrak{a}$ - идеал алгебры Ли $\mathcal{G}$. Тогда идеалом $U(\mathcal{G}) \mathfrak{a} U(\mathcal{G})$ универсальной обертывающей алгебры $U(\mathcal{G})$, порожденным $\mathfrak{a}$, является $U(\mathcal{G}) \mathfrak{a}=\mathfrak{a} U(\mathcal{G})$. Цепочка идеалов алгебры Ли $\mathfrak{u}_{n}$

$$
I_{1} \subset \cdots \subset I_{\lambda} \subset \cdots \subset \mathfrak{u}_{n}=I_{\operatorname{ord}\left(\Omega_{n}\right)}, \quad \lambda \in\left[1, \operatorname{ord}\left(\Omega_{n}\right)\right],
$$


дает цепочку подалгебр и цепочку идеалов алгебры $U_{n}$ соответственно

$$
U\left(I_{1}\right) \subset \cdots \subset U\left(I_{\lambda}\right) \subset \cdots \subset U_{n}, \quad U_{n} I_{1} \subset \cdots \subset U_{n} I_{\lambda} \subset \cdots \subset U_{n} .
$$

Пусть $U_{n, \lambda}^{\prime}:=\chi_{n}\left(U\left(I_{\lambda}\right)\right)$ и $I_{\lambda}^{\prime}:=\chi_{n}\left(U_{n} I_{\lambda}\right)$, где $\chi_{n}$ - гомоморфизм алгебр (2.11). Тогда

$$
U_{n, 1}^{\prime} \subseteq \cdots \subseteq U_{n, \lambda}^{\prime} \subseteq \cdots \subseteq U_{n}^{\prime}=\chi_{n}\left(U_{n}\right)
$$

является цепочкой подалгебр алгебры $U_{n}^{\prime}$, а

$$
I_{n, 1}^{\prime} \subseteq \cdots \subseteq I_{n, \lambda}^{\prime} \subseteq \cdots \subseteq U_{n}^{\prime}
$$

является цепочкой идеалов алгебры $U_{n}^{\prime}$.

ПреДлОженИЕ 3.13. 1) Все включения в (3.5) строгие. Алгебра $U_{n}^{\prime}$ не конечно порождена.

2) Все включения в (3.6) строгие. В частности, алгебра $U_{n}^{\prime}$ не является ни лево-, ни правонётеровой алгеброй и не удовлетворяет условию максимальности для идеалов.

ДокАЗАтельство. 1. Воспользуется индукцией по $n$. Пусть $n=2$. Для каждого $i \in[1, \omega)$ алгебра $U_{2, i}^{\prime}$ порождается коммутирующими элементами $x_{1}^{j} \partial_{2}$, где $0 \leqslant j \leqslant i-1$, так как

$$
x_{1}^{j} \partial_{2} \cdot x_{1}^{k} \partial_{2}=x_{1}^{j+k} \partial_{2}^{2} \quad \forall j, k .
$$

Эти равенства означают, что алгебра $U_{2, i}^{\prime}$ изоморфна моноидной алгебре $K \mathcal{M}_{i}$ (посредством $\left.x_{1}^{j} \partial_{2} \mapsto(j, 1)\right)$, где $\mathcal{M}_{i}-$ подмоноид из $\left(\mathbb{N}^{2},+\right)$, порожденный элементами $(j, 1), 0 \leqslant j \leqslant i-1$. Следовательно,

$$
x_{1}^{i-1} \partial_{2} \in U_{2, i}^{\prime} \backslash U_{2, i-1}^{\prime}, \quad i=2,3, \ldots .
$$

Это означает, что вложения $U_{2,1}^{\prime} \subset U_{2,2}^{\prime} \subset \cdots \subset U_{2, i}^{\prime} \subset \cdots \subset U_{2, \omega}^{\prime}=\bigcup_{i \geqslant 1} U_{2, i}^{\prime}$ строгие, и потому алгебра $U_{2, \omega}^{\prime}$ не конечно порождена. Поскольку $\partial_{1} \in U_{2}^{\prime} \backslash U_{2, \omega}^{\prime}$, $U_{2}^{\prime}=U_{2, \omega}^{\prime}\left[\partial_{1} ; \operatorname{ad}\left(\partial_{1}\right)\right]$ является алгеброй косых многочленов и $\left[\partial_{1}, U_{2, i}^{\prime}\right] \subseteq U_{2, i}^{\prime}$ для всех $i \geqslant 1$, утверждение 1) верно для $n=2$.

Предположим, что $n>2$ и утверждение 1) верно для всех $n^{\prime}<n$. Пусть $\lambda \in\left[1, \omega^{n-1}\right)$. Тогда $\lambda+1$ не является предельным ординалом. Заметим, что $\left[1, \omega^{n-1}\right) \subseteq\left[1, \operatorname{ord}\left(\Omega_{n}\right)\right)$. Отсюда следует, что $\lambda+1=(\alpha, n)$. Элементы $\left\{x^{\beta} \partial_{n} \mid\right.$ $\left.\beta \in \mathbb{N}^{n-1}\right\}$ перестановочны. Более того,

$$
x^{\beta} \partial_{n} \cdot x^{\gamma} \partial_{n}=x^{\beta+\gamma} \partial_{n}^{2}, \quad \beta, \gamma \in \mathbb{N}^{n-1} .
$$

Значит, $U_{n, \lambda}^{\prime}$ является коммутативной алгеброй, изоморфной моноидной алгебре $K \mathcal{M}_{n, \lambda}$ (посредством $\left.x^{\beta} \partial_{n} \mapsto(\beta, 1)\right)$, где $\mathcal{M}_{n, \lambda}-$ подмоноид из $\left(\mathbb{N}^{n},+\right)$, порожденный элементами $\{(\beta, 1) \mid(\beta, n) \leqslant \lambda\}$. Следовательно,

$$
x^{\alpha} \partial_{n} \in U_{n, \lambda+1}^{\prime} \backslash U_{n, \lambda}^{\prime} \quad \forall \lambda \in\left[1, \omega^{n-1}\right) .
$$

Это означает, что (включения строгие)

$$
U_{n, 1}^{\prime} \subset U_{n, 2}^{\prime} \subset \cdots \subset U_{n, \lambda}^{\prime} \subset \cdots \subset U_{n, \omega^{n-1}}^{\prime}=\bigcup_{\lambda \in\left[1, \omega^{n-1}\right)} U_{n, \lambda}^{\prime} .
$$


Пусть $\lambda \geqslant \omega^{n-1}$. В силу теоремы $\left.2.6,1\right)$ и $(3.3)$

$U_{n}^{\prime} / I_{n, \omega^{n-1}}^{\prime} \simeq U_{n-1}^{\prime}, \quad\left(U_{n, \omega^{n-1}+\mu}^{\prime}+I_{n, \omega^{n-1}}^{\prime}\right) / I_{n, \omega^{n-1}}^{\prime} \simeq U_{n-1, \mu}^{\prime}, \quad \mu \in\left[1, \operatorname{ord}\left(\Omega_{n-1}\right)\right]$.

В силу индукции по $n$ утверждение 1) выполняется.

2. Мы свободно используем факты, доказанные в п. 1. Воспользуемся индукцией по $n$. Пусть $n=2$. Для каждого $i \in[1, \omega)$ идеал $I_{2, i}^{\prime}$ алгебры $U_{2}^{\prime}$ порожден коммутирующими элементами $x_{1}^{j} \partial_{2}$, где $0 \leqslant j \leqslant i-1$. В силу следствия 2.7 множество $W_{2}^{\prime}=\left\{\partial_{1}^{i} \partial_{2}^{j}, \partial_{1}^{i} x_{1}^{k} \partial_{2} \cdot \partial_{2}^{j} \mid i, j \in \mathbb{N}, k \geqslant 1\right\}$ является $K$-базисом алгебры $U_{2}^{\prime}$. Для каждого $i \in[1, \omega)$ имеем

$$
I_{2, i}^{\prime}=\sum_{j=0}^{i-1} U_{2}^{\prime} x_{1}^{j} \partial_{2}=\bigoplus_{i \geqslant 0} \bigoplus_{k \geqslant 0, j \geqslant 2} K \partial_{1}^{i} x_{1}^{k} \partial_{2}^{j} \oplus \bigoplus_{i \geqslant 0}^{i-1} \bigoplus_{k=0}^{i} K \partial_{1}^{i} x_{1}^{k} \partial_{2} .
$$

Следовательно,

$$
x_{1}^{i-1} \partial_{2} \in I_{2, i}^{\prime} \backslash I_{2, i-1}^{\prime}, \quad i=2,3, \ldots
$$

Это означает, что

$$
I_{2,1}^{\prime} \subset I_{2,2}^{\prime} \subset \cdots \subset I_{2, i}^{\prime} \subset \cdots \subset I_{2, \omega}^{\prime}=\bigcup_{i \geqslant 1} I_{2, i}^{\prime} .
$$

Поскольку $1 \in U_{2}^{\prime} \backslash I_{2, \omega+1}^{\prime}$ и $\partial_{1} \in I_{2, \omega+1}^{\prime} \backslash I_{2, \omega}^{\prime}$, утверждение 2) верно для $n=2$.

Предположим, что $n>2$ и утверждение 2) верно для всех $n^{\prime}<n$. Пусть $\lambda \in\left[1, \omega^{n-1}\right)$. Тогда $\lambda+1$ не является предельным ординалом. Заметим, что $\left[1, \omega^{n-1}\right) \subseteq\left[1, \operatorname{ord}\left(\Omega_{n}\right)\right)$. Отсюда следует, что $\lambda+1=(\alpha, n)$. Из равенства $I_{n, \lambda+1}^{\prime}=U_{n}^{\prime} \chi_{n}\left(I_{\lambda+1}\right)=\sum_{(\beta, n) \leqslant \lambda+1} U_{n}^{\prime} x^{\beta} \partial_{n}$ и $(2.2)$ следует, что

$$
I_{n, \lambda+1}^{\prime} \cap\left(\bigoplus_{\beta \in \mathbb{N}^{n-1}} K x^{\beta} \partial_{n}\right)=\bigoplus_{(\beta, n) \leqslant \lambda+1} K x^{\beta} \partial_{n}
$$

Следовательно,

$$
x^{\alpha} \partial_{n} \in I_{n, \lambda+1}^{\prime} \backslash I_{n, \lambda}^{\prime} \quad \forall \lambda \in\left[1, \omega^{n-1}\right) .
$$

Это означает, что

$$
I_{n, 1}^{\prime} \subset I_{n, 2}^{\prime} \subset \cdots \subset I_{n, \lambda}^{\prime} \subset \cdots \subset I_{n, \omega^{n-1}}^{\prime}=\bigcup_{\lambda \in\left[1, \omega^{n-1}\right)} I_{n, \lambda}^{\prime} .
$$

Пусть $\lambda>\omega^{n-1}$. Ввиду (3.7) также имеем

$$
I_{n, \omega^{n-1}+\mu}^{\prime} / I_{n, \omega^{n-1}}^{\prime} \simeq I_{n-1, \mu}^{\prime} \quad \forall \mu \in\left[1, \operatorname{ord}\left(\Omega_{n-1}\right)\right] .
$$

В силу индукции по $n$ утверждение 2) выполняется. Предложение доказано.

3.7. Подалгебры Ли Гейзенберга алгебры $\mathfrak{u}_{n}$. Пусть

$$
\mathrm{gl}_{n}(K)=\bigoplus_{i, j=1}^{n} K E_{i j}
$$

- алгебра Ли матриц размера $n \times n$, где $E_{i j}$ - матричные единицы. Для каждого $n \geqslant 2$ пусть $\operatorname{UT}_{n}(K)=\bigoplus_{i \leqslant j} K E_{i j}$ - алгебра Ли верхнетреугольных матриц 
размера $n \times n, \mathrm{UT}_{n}(K) \subseteq \mathrm{gl}_{n}(K) ; K$-линейное отображение

$$
\mathrm{UT}_{n}(K) \rightarrow \mathfrak{u}_{n}, \quad E_{i j} \mapsto x_{i} \partial_{j},
$$

является мономорфизмом алгебр Ли. Отождествим алгебру Ли $\mathrm{UT}_{n}(K)$ с ее образом в $\mathfrak{u}_{n}$.

Алгебра Ли Гейзенберга $\mathcal{H}_{n}$ - это $(2 n+1)$-мерная алгебра Ли с $K$-базисом $X_{1}, \ldots, X_{n}, Y_{1}, \ldots, Y_{n}, Z$, в которой $Z$ - центральный элемент алгебры $\mathcal{H}_{n}$,

$$
\left[Y_{i}, X_{j}\right]=\delta_{i j} Z, \quad\left[X_{i}, X_{j}\right]=0, \quad\left[Y_{i}, Y_{j}\right]=0, \quad i, j=1, \ldots, n,
$$

где $\delta_{i j}$ - дельта-функция Кронекера. $K$-линейное отображение

$$
\mathcal{H}_{n-1} \rightarrow \mathfrak{u}_{n}, \quad X_{i} \mapsto x_{i} \partial_{n}, \quad Y_{i} \mapsto \partial_{i}, \quad Z \mapsto \partial_{n}, \quad i=1, \ldots, n-1,
$$

является мономорфизмом алгебр Ли. Отождествим алгебру Ли Гейзенберга $\mathcal{H}_{n-1}$ с ее образом в $\mathfrak{u}_{n}$. Поскольку $\mathcal{H}_{i-1} \subseteq \mathfrak{u}_{i} \subseteq \mathfrak{u}_{n}$ для $i=2, \ldots, n-1$, алгебра Ли $\mathfrak{u}_{n}$ содержит алгебры Ли Гейзенберга $\mathcal{H}_{i}, i=1, \ldots, n-1$. Для всех натуральных чисел $i$ и $j, i \neq j$, имеем $\mathcal{H}_{i} \cap \mathcal{H}_{j}=\sum_{k=1}^{\min \{i, j\}} K \partial_{k}$.

Пусть $A$ - алгебра или алгебра Ли, а $M-$ ее модуль. Тогда $\operatorname{ann}_{A}(M):=\{a \in$ $A \mid a M=0\}$ называется аннулятором $A$-модуля $M$. Это идеал алгебры $A$. Модуль называется точным, если его аннулятор равен нулю.

3.8. $\mathfrak{u}_{n}$-модуль $P_{n}$. Утверждать, что $P_{n}$ является $\mathfrak{u}_{n}$-модулем - это то же, что утверждать, что $P_{n}$ является $U_{n}^{\prime}$-модулем. Важность этого очевидного наблюдения состоит в том, что соотношения алгебры Вейля $A_{n}$ можно использовать в различных вычислениях в алгебре $U_{n}^{\prime}$, так как $U_{n}^{\prime} \subseteq A_{n}$. Для каждого $n \geqslant 2$ алгебра Ли $\mathfrak{u}_{n}$ является подалгеброй Ли алгебры Ли $\mathfrak{u}_{n+1}=\mathfrak{u}_{n} \oplus P_{n} \partial_{n+1}$, $P_{n} \partial_{n+1}$ - идеал алгебры Ли $\mathfrak{u}_{n+1}$ и $\left[P_{n} \partial_{n+1}, P_{n} \partial_{n+1}\right]=0$. В частности, $P_{n} \partial_{n+1}-$ левый $\mathfrak{u}_{n}$-модуль, где действие алгебры Ли $\mathfrak{u}_{n}$ на $P_{n} \partial_{n+1}$ задается по правилу $u v:=[u, v]$ для всех $u \in \mathfrak{u}_{n}$ и $v \in P_{n} \partial_{n+1}$. Напомним, что алгебра многочленов $P_{n}$ является левым $\mathfrak{u}_{n}$-модулем.

Лемма 3.14. 1) K-линейное отображение $P_{n} \rightarrow P_{n} \partial_{n+1}, p \mapsto p \partial_{n+1}$, является изоморбизмом $\mathfrak{u}_{n}$-модулей.

2) $\mathfrak{u}_{n}$-модуль $P_{n}$ является неразложимым однорядным $\mathfrak{u}_{n}$-модулем, кроме mого, u. $\operatorname{dim}\left(P_{n}\right)=\omega^{n} u \operatorname{ann}_{\mathfrak{u}_{n}}\left(P_{n}\right)=0$.

3) Множество $\left\{P_{\lambda, n}:=\bigoplus_{\alpha \in \mathbb{N}^{n},(\alpha, n+1) \leqslant \lambda} K x^{\alpha} \mid \lambda \in\left[1, \omega^{n}\right]\right\}$ является множеством всех ненулевых $\mathfrak{u}_{n}$-подмодулей из $P_{n} ; P_{\lambda, n} \subset P_{\mu, n}$ тогда и только тогда, когда $\lambda<\mu$; u. $\operatorname{dim}\left(P_{\lambda, n}\right)=\lambda$ для всех $\lambda \in\left[1, \omega^{n}\right]$.

4) Все $\mathfrak{u}_{n}$-подмодули из $P_{n}$ являются попарно неизоморбными неразложимыми однорядными $\mathfrak{u}_{n}-$ модулями.

ДоказАТЕЛЬство. Для доказательства утверждения 1) отметим, что данное отображение является биекцией и $\mathfrak{u}_{n}$-гомоморфизмом.

Докажем утверждение 3). В силу утверждения 1) можно отождествить $\mathfrak{u}_{n}$-модуль $P_{n}$ с идеалом $P_{n} \partial_{n+1}$ алгебры Ли $\mathfrak{u}_{n+1}$. При этом отождествлении каждый $\mathfrak{u}_{n}$-подмодуль из $P_{n}$ становится идеалом алгебры Ли $\mathfrak{u}_{n+1}$ в $P_{n} \partial_{n+1}$, и наоборот. Теперь утверждение 3) следует из утверждения 1) и классификации идеалов алгебры Ли $\mathfrak{u}_{n+1}$ (теорема $\left.3.6,1\right)$ ). 
Теперь докажем утверждение 2). В силу утверждения 3) $\mathfrak{u}_{n}$-модуль $P_{n}$ одноряден, следовательно, неразложим и u. $\operatorname{dim}\left(P_{n}\right)=\omega^{n}$. Алгебра Вейля $A_{n}$ является простой алгеброй, отсюда следует, что $\operatorname{ann}_{A_{n}}\left(P_{n}\right)=0$. Тогда $\operatorname{ann}_{\mathfrak{u}_{n}}\left(P_{n}\right)=\mathfrak{u}_{n} \cap \operatorname{ann}_{A_{n}}\left(P_{n}\right)=0$, так как $\mathfrak{u}_{n} \subseteq A_{n}$.

Докажем утверждение 4). В силу утверждения 3) имеем u. $\operatorname{dim}\left(P_{\lambda, n}\right)=\lambda$. Отсюда следует, что $P_{\lambda, n} \nsucceq P_{\mu, n}$ для всех $\lambda \neq \mu$. Остальное очевидно (см. утверждение 2)).

Следующее следствие описывает аннуляторы всех $\mathfrak{u}_{n}$-подмодулей из $P_{n}$. В частности, оно классифицирует точные $\mathfrak{u}_{n}$-подмодули $\mathfrak{u}_{n}$-модуля $P_{n}$.

СледСТвИЕ 3.15. 1) $\mathfrak{u}_{n}$-подмодуль $P_{\lambda, n}$ из $P_{n}$ является точным подмодулем тогда и только тогда, когда $\lambda \in\left[\omega^{n-1}+1, \omega^{n}\right]$.

2) Имеем $\operatorname{ann}_{\mathfrak{u}_{n}}\left(P_{\omega^{n-1}, n}\right)=P_{n-1} \partial_{n}$.

3) Имеем $\operatorname{ann}_{\mathfrak{u}_{n}}\left(P_{\lambda, n}\right)=\left\{\begin{array}{lll}0, & \text { если } & \lambda \in\left(\omega^{n-1}, \omega^{n}\right], \\ \bigoplus_{i=m+1}^{n} P_{i-1} \partial_{i}, & \text { если } & \lambda \in\left(\omega^{m-1}, \omega^{m}\right], \\ \mathfrak{u}_{n}, & & m=1, \ldots, n-1, \\ & \text { если } & \lambda=1 .\end{array}\right.$

ДокАзАтельство. Сначала докажем утверждение 2). Вложение $\supseteq$ очевидно. Обратное вложение $\subseteq$ следует из того, что $P_{n-1}-$ точный $\mathfrak{u}_{n-1}$-модуль (лемма $3.14,2)), \mathfrak{u}_{n-1} \simeq \mathfrak{u}_{n} / P_{n-1} \partial_{n}$ и $P_{n-1}=P_{\omega^{n-1}, n}$ является $\left(\mathfrak{u}_{n} / P_{n-1} \partial_{n}\right)$ модулем.

Для доказательства утверждения 1) заметим, что если $N$ - подмодуль из $M$, то $\operatorname{ann}(N) \supseteq \operatorname{ann}(M)$. Ввиду этого факта и утверждения 2), чтобы завершить доказательство утверждения 1$)$, достаточно показать, что $\mathfrak{u}_{n}$-модуль $P_{\omega^{n-1}+1, n}$ точен. Поскольку $P_{\omega^{n-1}, n} \subseteq P_{\omega^{n-1}+1, n}$, имеем $\mathfrak{a}:=\operatorname{ann}_{\mathfrak{u}_{n}}\left(P_{\omega^{n-1}+1, n}\right) \subseteq$ $\operatorname{ann}_{\mathfrak{u}_{n}}\left(P_{\omega^{n-1}, n}\right)=P_{n-1} \partial_{n}$. Так как $x_{n} \in P_{\omega^{n-1}+1, n}$ и $p \partial_{n} *\left(x_{n}\right)=p \neq 0$ для всех ненулевых элементов $p \in P_{n-1}$, имеем $\mathfrak{a}=0$.

Докажем утверждение 3 ). Воспользуемся индукцией по $n \geqslant 2$. Начальный случай состоит в том, что $n=2$, и нужно рассмотреть три случая: $\lambda \in\left(\omega, \omega^{2}\right]$, $\lambda=(1, \omega]$ и $\lambda=1$. Первый случай - это утверждение 1$)$. Последний случай очевиден. Во втором случае имеем

$$
\operatorname{ann}_{\mathfrak{u}_{2}}\left(P_{m, 2}\right)=\operatorname{ann}_{\mathfrak{u}_{2}}\left(\bigoplus_{i=0}^{m-1} K x_{1}^{i}\right)=K\left[x_{1}\right] \partial_{2} .
$$

Пусть $n>2$, и предположим, что утверждение 3) выполнено для всех $n^{\prime}<n$. Если $\lambda \in\left(\omega^{n-1}, \omega^{n}\right]$, то $\mathfrak{u}_{n}$-модуль $P_{\lambda, n}$ точен по утверждению 1$)$. Если $\lambda=1$, то $\operatorname{ann}_{\mathfrak{u}_{n}}\left(P_{1, n}\right)=\operatorname{ann}_{\mathfrak{u}_{n}}(K)=\mathfrak{u}_{n}$. Если $1<\lambda \leqslant \omega^{n-1}$, т. е. $\lambda \in\left(\omega^{m-1}, \omega^{m}\right]$ для некоторого $m \in\{1, \ldots, n-1\}$, то $P_{\lambda, n} \subseteq P_{n-1}$. В силу утверждения 2$)$ имеем $\mathfrak{a}:=$ $\operatorname{ann}_{\mathfrak{u}_{n}}\left(P_{\lambda, n}\right) \supseteq \operatorname{ann}_{\mathfrak{u}_{n}}\left(P_{\omega^{n-1}, n}\right)=P_{n-1} \partial_{n}$. Поскольку $\mathfrak{u}_{n-1} \simeq \mathfrak{u}_{n} / P_{n-1} \partial_{n}$, вложение $P_{\lambda, n} \subseteq P_{n-1}$ является вложением $\mathfrak{u}_{n-1}$-модулей. Более того, $\mathfrak{u}_{n-1}$-подмодуль $P_{\lambda, n}$ из $P_{n-1}$ можно отождествить с $\mathfrak{u}_{n-1}$-подмодулем $P_{\lambda, n-1}$ из $P_{n-1}$. Теперь результат следует индукцией по $n$. 
СлЕДСТВИЕ 3.16. При $n \geqslant 3$ идеал $I_{\omega^{n-2}+1}=K x_{n-1} \partial_{n}+\sum_{\alpha \in \mathbb{N}^{n-2}} K x^{\alpha} \partial_{n}$ является наименьшим идеалом I алгебры Ли $\mathfrak{u}_{n}$, который является точным $\mathfrak{u}_{n-1}$-модулем $\left(\right.$ m.e. $\left.\operatorname{ann}_{\mathfrak{u}_{n-1}}(I)=0\right)$.

ДокАЗАТЕЛЬСтво. В силу леммы $3.14,1)$ и следствия $3.15,1) \mathfrak{u}_{n-1}$-модуль $I_{\omega^{n-2}+1}$ точен, но его предшественник $I_{\omega^{n-2}}$ не точен, так как $\operatorname{ann}_{\mathfrak{u}_{n-1}}\left(I_{\omega^{n-2}}\right)=$ $\operatorname{ann}_{\mathfrak{u}_{n-1}}\left(\sum_{\alpha \in \mathbb{N}^{n-2}} K x^{\alpha} \partial_{n}\right) \ni \partial_{n-1}$. Следствие доказано.

Вложение алгебр Ли $\mathfrak{u}_{n} \subseteq \mathfrak{u}_{n+1}=\mathfrak{u}_{n} \oplus P_{n-1} \partial_{n}$ сохраняет линейные упорядочения на базисах $\mathcal{B}_{n}$ и $\mathcal{B}_{n+1}$. Изоморфизм $\mathfrak{u}_{n}$-модулей $P_{n} \rightarrow P_{n} \partial_{n+1}$, $p \mapsto p \partial_{n+1}$ (лемма $\left.3.14,1\right)$ ) индуцирует линейное упорядочение на мономах $\left\{x^{\alpha}\right\}_{\alpha \in \mathbb{N}^{n}}$ алгебры многочленов $P_{n}$ по правилу $x^{\alpha}>x^{\beta}$ тогда и только тогда, когда $X_{\alpha, n+1}>X_{\beta, n+1}$, т. е. тогда и только тогда, когда $\alpha_{n}=\beta_{n}$, $\alpha_{n-1}=\beta_{n-1}, \ldots, \alpha_{m+1}=\beta_{m+1}$ и $\alpha_{m}>\beta_{m}$ для некоторого $m \in\{1, \ldots, n\}$. Это так называемое обратное лексикографическое упорядочение на $\left\{x^{\alpha}\right\}_{\alpha \in \mathbb{N}^{n}}$ или $\mathbb{N}^{n}\left(\alpha>\beta\right.$ тогда и только тогда, когда $\left.x^{\alpha}>x^{\beta}\right)$. В силу леммы $\left.\left.3.4,3\right), 4\right)$ и леммы $3.14,1$ ) если $x^{\alpha}>x^{\beta}$ (где $\alpha, \beta \in \mathbb{N}^{n}$ ), то:

(i) $\partial_{k} * x^{\alpha}>\partial_{k} * x^{\beta}$ для всех $k=1, \ldots, n$ таких, что $\alpha_{k} \neq 0$;

(ii) $X_{\gamma, k} * x^{\alpha}>X_{\gamma, k} * x^{\beta}$ для всех $k=1, \ldots, n-1$ и $\gamma \in \mathbb{N}^{k-1}$ таких, что $X_{\gamma, k} * x^{\alpha} \neq 0$, т. е. $\alpha_{k} \neq 0$.

Пусть $\mathcal{M}\left(P_{n}\right)$ - множество всех ненулевых подмодулей $\mathfrak{u}_{n}$-модуля $P_{n}$; это линейно упорядоченное множество относительно $\subseteq$. В силу леммы $3.14,3$ ) отображение

$$
\kappa_{n}:\left[1, \omega^{n}\right] \rightarrow \mathcal{M}\left(P_{n}\right), \quad \lambda \mapsto P_{\lambda, n},
$$

является изоморфизмом линейно упорядоченных множеств. Каждый ненулевой многочлен $p \in P_{n}$ имеет однозначное представление в виде суммы

$$
\lambda_{\alpha} x^{\alpha}+\sum_{\alpha>\beta} \lambda_{\beta} x^{\beta}, \quad \lambda_{\alpha} \in K^{*}, \quad \lambda_{\beta} \in K .
$$

Элементы $\lambda_{\alpha} x^{\alpha}$ и $\lambda_{\alpha}$ называются старшим членом и старшим коэффициентом многочлена $p$ соответственно (относительно линейного упорядочения $>$ ). Ординальное число

$$
\operatorname{ord}(p):=\operatorname{ord}([1, \alpha])
$$

$\left(\right.$ где $\left.[1, \alpha] \subseteq\left[1, \omega^{n}\right]\right)$ называется ординальной степенъю многочлена $p$. Для всех ненулевых многочленов $p, q \in P_{n}$ и для $\lambda \in K^{*}$ имеем:

(i) $\operatorname{ord}(p+q) \leqslant \max \{\operatorname{ord}(p)$, ord $(q)\}$, как только $p+q \neq 0$;

(ii) $\operatorname{ord}(\lambda p)=\operatorname{ord}(p)$;

(iii) $\operatorname{ord}(u * p)<\operatorname{ord}(p)$ для всех $u \in \mathfrak{u}_{n}$ таких, что $u * p \neq 0$.

Для каждого ординала $\lambda \in\left[1, \omega^{n}\right)$ имеется однозначное представление:

$$
\lambda=\alpha_{n} \omega^{n-1}+\alpha_{n-1} \omega^{n-2}+\cdots+\alpha_{2} \omega+\alpha_{1},
$$

где $\alpha_{i} \in \mathbb{N}$ и не все $\alpha_{j}$ равны нулю (отметим сдвиг на 1 в индексах коэффициентов $\left.\alpha_{i}\right)$. Тогда

$$
P_{\lambda, n}=\left\{\begin{array}{lll}
\oplus\left\{K x^{\beta} \mid \beta \in \mathbb{N}^{n}, x^{\beta} \leqslant x_{1}^{-1} x^{\alpha}\right\}, & \text { если } & \alpha_{1} \neq 0 \\
\oplus\left\{K x^{\beta} \mid \beta \in \mathbb{N}^{n}, x^{\beta}<x^{\alpha}\right\}, & \text { если } & \alpha_{1}=0
\end{array}\right.
$$


где $x^{\alpha}=\prod_{i=1}^{n} x_{i}^{\alpha_{i}}$. Заметим, что $\alpha_{1} \neq 0$ тогда и только тогда, когда $\lambda-$ непредельный ординал. Векторное пространство $P_{\lambda, n}$ обладает наибольшим мономом тогда и только тогда, когда $\lambda$ - непредельный ординал, и в этом случае $x_{1}^{-1} x^{\alpha}-$ наибольший моном из $P_{\lambda, n}$. Ординальное число $\lambda \in\left[1, \omega^{n}\right)$ однозначно представляется в виде суммы:

$$
\lambda=\alpha_{m} \omega^{m-1}+\alpha_{m-1} \omega^{m-2}+\cdots+\alpha_{j} \omega^{j-1}, \quad \alpha_{m} \neq 0, \quad \alpha_{j} \neq 0,
$$

где $\alpha_{i} \in \mathbb{N}, 1 \leqslant m \leqslant n$ и $1 \leqslant j \leqslant m$. Натуральные числа $\alpha_{m}$ и $\alpha_{j}$ называются кратностъю и кократностъю ординального числа $\lambda$. Натуральные числа $m-1$ и $j-1$ называются степенъю и костепенъю ординального числа $\lambda$.

Следующая лемма очевидна.

ЛЕмма 3.17. Пусть $\lambda \in\left[1, \omega^{n}\right)$, m.e. $\lambda=\alpha_{m} \omega^{m-1}+\alpha_{m-1} \omega^{m-2}+\cdots$ $\cdots+\alpha_{j} \omega^{j-1}$, где $\alpha_{i} \in \mathbb{N}, \alpha_{m} \neq 0, \alpha_{j} \neq 0, m \leqslant n u j \geqslant 1$. Тогда

$$
\begin{gathered}
P_{\lambda, n}=\sum_{i=0}^{\alpha_{m}-1} x_{m}^{i} P_{m-1}+x_{m}^{\alpha_{m}} \sum_{0 \leqslant i \leqslant \alpha_{m-1}-1} x_{m-1}^{i} P_{m-2}+\cdots \\
\cdots+x_{m}^{\alpha_{m}} x_{m-1}^{\alpha_{m-1}} \cdots x_{k+1}^{\alpha_{k+1}} \sum_{0 \leqslant i \leqslant \alpha_{k}-1} x_{k}^{i} P_{k-1}+\cdots \\
\cdots+x_{m}^{\alpha_{m}} x_{m-1}^{\alpha_{m-1}} \cdots x_{j+1}^{\alpha_{j+1}} \sum_{0 \leqslant i \leqslant \alpha_{j}-1} x_{j}^{i} P_{j-1} .
\end{gathered}
$$

B частности, $P_{\alpha_{m} \omega^{m-1}, n}=\sum_{i=0}^{\alpha_{m}-1} x_{m}^{i} P_{m-1}$.

ЗАмЕчАниЕ 3.18. Если $\alpha_{k}=0$, то соответствующее слагаемое отсутствует.

Пусть $V$ - векторное пространство над полем $K$. Линейное отображение $\varphi: V \rightarrow V$ называется отображением/оператором Фредгольма, если его ядро и коядро конечномерны, и тогда

$$
\operatorname{ind}(\varphi):=\operatorname{dim}_{K}(\operatorname{ker}(\varphi))-\operatorname{dim}_{K}(\operatorname{coker}(\varphi))
$$

называется индексом отображения $\varphi$. Пусть $\mathcal{F}(V)$ - множество всех линейных отображений Фредгольма в $V$. На самом деле это моноид, так как

$$
\operatorname{ind}(\varphi \psi)=\operatorname{ind}(\varphi)+\operatorname{ind}(\psi) \quad \forall \varphi, \psi \in \mathcal{F}(V) .
$$

Пусть $V$ - векторное пространство со счетным $K$-базисом $\left\{e_{i}\right\}_{i \in \mathbb{N}}$, а $\partial-$ $K$-линейное отображение на $V$, заданное по правилу $\partial e_{i}=e_{i-1}$ для всех $i \in \mathbb{N}$, где $e_{-1}:=0$.

Например, $V=K[x], e_{i}:=\frac{x^{i}}{i !}$ и $\partial=\frac{d}{d x}$. Подалгебра из $\operatorname{End}_{K}(V)$, порожденная отображением $\partial$, является алгеброй многочленов $K[\partial]$. Поскольку отображение $\partial$ локально нильпотентно, алгебра $\operatorname{End}_{K}(V)$ содержит алгебру $K[[\partial]]=\left\{\sum_{i=0}^{\infty} \lambda_{i} \partial^{i} \mid \lambda_{i} \in K\right\}$ формальных степенных рядов от $\partial$. Множество $K[[\partial]]^{*}=\left\{\sum_{i=0}^{\infty} \lambda_{i} \partial^{i} \in K[[\partial]] \mid \lambda_{0} \in K^{*}\right\}$ - группа единиц алгебры $K[[\partial]]$. Векторное пространство $V$ является $K[\partial]$-модулем. 
Следующая лемма тривиальна.

Лемма 3.19. 1) Имеем

$$
\begin{array}{r}
\operatorname{End}_{K[\partial]}(V)=\left\{\varphi \in \operatorname{End}_{K}(V), \varphi\left(e_{i}\right)=\sum_{j=0}^{i} \lambda_{j} e_{i-j}=\left(\sum_{j \geqslant 0} \lambda_{j} \partial^{j}\right)\left(e_{i}\right), i \in \mathbb{N} \mid\right. \\
\left.\lambda_{i} \in K, i \in \mathbb{N}\right\}=K[[\partial]],
\end{array}
$$

$$
\begin{array}{r}
\operatorname{Aut}_{K[\partial]}(V)=\left\{\varphi \in \operatorname{End}_{K}(V), \varphi\left(e_{i}\right)=\sum_{j=0}^{i} \lambda_{j} e_{i-j}, i \in \mathbb{N} \mid\right. \\
\left.\quad \lambda_{0} \neq 0, \lambda_{i} \in K, i \in \mathbb{N}\right\}=K[[\partial]]^{*} .
\end{array}
$$

2) В частности, все ненулевые элементы $\operatorname{End}_{K[\partial]}(V)$ являются сюргективными отображениями Фредгольма.

3) Пусть $a=\sum_{i \geqslant d} \lambda_{i} \partial^{i} \in K[[\partial]] u \lambda_{d} \neq 0$. Тогда $d=\operatorname{ind}(a)=\operatorname{dim}_{K}(\operatorname{ker}(a))$.

Пусть $V$ - векторное пространство с $K$-базисом $\left\{e_{i}\right\}_{i \in \mathbb{N}}$, а $x-K$-линейное отображение на $V$, определенное следующим образом: $x e_{i}:=e_{i+1}$ для всех $i \in \mathbb{N}$. Например, $V=K[x], e_{i}:=x^{i}$ и $x: K[x] \rightarrow K[x], p \mapsto x p$. Алгебра многочленов $K[x]$ является подалгеброй алгебры $\operatorname{End}_{K}(V)$.

Следующая лемма очевидна.

Лемма 3.20. 1) Имеем $\operatorname{End}_{K[x]}(V)=\{p: V \rightarrow V, a \mapsto p a \mid p \in K[x]\} \simeq$ $K[x]$ (посредством $p \mapsto p)$. В частности, все ненулевые элементы $\operatorname{End}_{K[x]}(V)$ являются ингективными отображениями.

2) Имеем $\operatorname{Aut}_{K[x]}(V)=K^{*}$.

3) Для всех $p \in \operatorname{End}_{K[x]}(V)=K[x]$ выполнены равенства $\operatorname{deg}(p)=-\operatorname{ind}(p)=$ $\operatorname{dim}_{K}(\operatorname{coker}(p))$.

Следующее предложение описывает алгебру всех $\mathfrak{u}_{n}$-гомоморфизмов (и ее группу единиц) $\mathfrak{u}_{n}$-модуля $P_{n}$. Ясно, что $K\left[x_{n}\right] \subseteq P_{n}-$ вложение $K\left[\partial_{n}\right]$-модулей и $K\left[\left[\frac{d}{d x_{n}}\right]\right]=\operatorname{End}_{K\left[\partial_{n}\right]}\left(K\left[x_{n}\right]\right)$ (лемма $\left.\left.3.19,1\right)\right) . K$-дифференцирование $\frac{d}{d x_{n}}$ алгебры многочленов $K\left[x_{n}\right]$ также обозначается через $\partial_{n}$.

ПРЕДЛОЖЕНИЕ 3.21. 1) Отображсение $\operatorname{End}_{\mathfrak{u}_{n}}\left(P_{n}\right) \rightarrow \operatorname{End}_{K\left[\partial_{n}\right]}\left(K\left[x_{n}\right]\right)=$ $K\left[\left[\frac{d}{d x_{n}}\right]\right],\left.\varphi \mapsto \varphi\right|_{K\left[x_{n}\right]}$, является изоморфизмом $K$-алгебр с обратным отображением $\varphi^{\prime} \mapsto \varphi$, где $\varphi\left(x^{\beta} x_{n}^{i}\right):=X_{\beta, n} \varphi^{\prime}\left(\frac{x_{n}^{i+1}}{i+1}\right)$ для всех $\beta \in \mathbb{N}^{n-1} u i \in \mathbb{N}$.

2) Отображение $\operatorname{Aut}_{\mathfrak{u}_{n}}\left(P_{n}\right) \rightarrow \operatorname{Aut}_{K\left[\partial_{n}\right]}\left(K\left[x_{n}\right]\right)=K\left[\left[\frac{d}{d x_{n}}\right]\right]^{*},\left.\varphi \mapsto \varphi\right|_{K\left[x_{n}\right]}$, является групповым изоморфизмом с обратным отображением, как в утверждении 1).

3) Каждое ненулевое отображение $\varphi \in \operatorname{End}_{\mathfrak{u}_{n}}\left(P_{n}\right)$ является сюорбективным отображением с ядром $\operatorname{ker}(\varphi)=\bigoplus_{i=0}^{d-1} P_{n-1} x_{n}^{i}$, где $d=\operatorname{ind}\left(\left.\varphi\right|_{K\left[x_{n}\right]}\right)=$ $\operatorname{dim}_{K}\left(\operatorname{ker}\left(\left.\varphi\right|_{K\left[x_{n}\right]}\right)\right)$.

4) Для всех натуральных $d \geqslant 1$ выполняется $\left(\frac{\partial}{\partial x_{n}}\right)^{d} \in \operatorname{End}_{\mathfrak{u}_{n}}\left(P_{n}\right) u$ $\operatorname{ker}_{P_{n}}\left(\frac{\partial}{\partial x_{n}}\right)^{d}=\bigoplus_{i=0}^{d-1} P_{n-1} x_{n}^{i}$. В частности, $\left(\frac{\partial}{\partial x_{n}}\right)^{d}: P_{n} / \bigoplus_{i=0}^{d-1} P_{n-1} x_{n}^{i} \rightarrow P_{n}-$ изоморфизм и $\mathfrak{n}_{n}$-модулей. 
ДокАЗАтельство. Сначала докажем утверждение 1). В силу леммы 3.19 имеем $\operatorname{End}_{K\left[\partial_{n}\right]}\left(K\left[x_{n}\right]\right)=K\left[\left[\frac{d}{d x_{n}}\right]\right]$. Поскольку $K\left[x_{n}\right]=\bigcap_{i=1}^{n-1} \operatorname{ker}_{P_{n}}\left(\partial_{i}\right)$ и отображения $\partial_{1}, \ldots, \partial_{n}$ перестановочны, отображение $\left.\varphi \mapsto \varphi\right|_{K\left[x_{n}\right]}-$ корректно определенный гомоморфизм $K$-алгебр. Для всех элементов $\beta \in \mathbb{N}^{n-1}$ и $i \in \mathbb{N}$

$$
\varphi\left(x^{\beta} x_{n}^{i}\right)=\varphi\left(X_{\beta, n} * \frac{x_{n}^{i+1}}{i+1}\right)=X_{\beta, n} \varphi\left(\frac{x_{n}^{i+1}}{i+1}\right) .
$$

Значит, отображение ограничения является мономорфизмом. Чтобы доказать, что отображение ограничения - сюръекция, необходимо показать, что для данного отображения $\varphi^{\prime} \in \operatorname{End}_{K\left[\partial_{n}\right]}\left(K\left[x_{n}\right]\right)$ его продолжение $\varphi$, определенное, как в формулировке утверждения 1 ), является $\mathfrak{u}_{n}$-гомоморфизмом. Отображение $\varphi$ является $K$-линейным отображением, так что надо проверить, что $\varphi X_{\alpha, i}=X_{\alpha, i} \varphi$ для всех $i=1, \ldots, n$ и $\alpha \in \mathbb{N}^{i-1}$.

Случай 1: $i<n$. Для всех $\beta \in \mathbb{N}^{n-1}$ и $j \in \mathbb{N}$ имеем

$$
\begin{aligned}
\varphi X_{\alpha, i}\left(x^{\beta} \cdot x_{n}^{j}\right)= & \varphi\left(x^{\alpha} \partial_{i} * x^{\beta} x_{n}^{j}\right)=\varphi\left(\beta_{i} x^{\alpha+\beta-e_{i}} x_{n}^{j}\right)=\beta_{i} X_{\alpha+\beta-e_{i}, n} \varphi^{\prime}\left(\frac{x_{n}^{j+1}}{j+1}\right), \\
X_{\alpha, i} \varphi\left(x^{\beta} \cdot x_{n}^{j}\right)= & x^{\alpha} \partial_{i} *\left(x^{\beta} \partial_{n} * \varphi^{\prime}\left(\frac{x_{n}^{j+1}}{j+1}\right)\right)=\beta_{i} x^{\alpha+\beta-e_{i}} \partial_{n} * \varphi^{\prime}\left(\frac{x_{n}^{j+1}}{j+1}\right) \\
& \quad+x^{\beta} \partial_{n} * x^{\alpha} \partial_{i} * \varphi^{\prime}\left(\frac{x_{n}^{j+1}}{j+1}\right)=\beta_{i} x^{\alpha+\beta-e_{i}} \partial_{n} * \varphi^{\prime}\left(\frac{x_{n}^{j+1}}{j+1}\right)+0 \\
= & \beta_{i} X_{\alpha+\beta-e_{i}, n} \varphi^{\prime}\left(\frac{x_{n}^{j+1}}{j+1}\right) .
\end{aligned}
$$

Случай 2: $i=n$. Пусть $\beta \in \mathbb{N}^{n-1}$ и $j \in \mathbb{N}$. Предположим, что $j \geqslant 1$; тогда

$$
\begin{gathered}
\varphi X_{\alpha, n}\left(x^{\beta} x_{n}^{j}\right)=\varphi\left(x^{\alpha+\beta} j x_{n}^{j-1}\right)=X_{\alpha+\beta, n} \varphi^{\prime}\left(x_{n}^{j}\right), \\
X_{\alpha, n} \varphi\left(x^{\beta} x_{n}^{j}\right)=x^{\alpha} \partial_{n} *\left(x^{\beta} \partial_{n} * \varphi^{\prime}\left(\frac{x_{n}^{j+1}}{j+1}\right)\right)=x^{\alpha+\beta} \partial_{n} * \partial_{n} * \varphi^{\prime}\left(\frac{x_{n}^{j+1}}{j+1}\right) \\
=X_{\alpha+\beta, n} \varphi^{\prime}\left(\partial_{n} * \frac{x_{n}^{j+1}}{j+1}\right)=X_{\alpha+\beta, n} \varphi^{\prime}\left(x_{n}^{j}\right) .
\end{gathered}
$$

Предположим, что $j=0$; тогда

$$
\begin{gathered}
\varphi X_{\alpha, n}\left(x^{\beta}\right)=\varphi(0)=0, \\
X_{\alpha, n} \varphi\left(x^{\beta}\right)=x^{\alpha} \partial_{n} * x^{\beta} \partial_{n} * \varphi^{\prime}\left(x_{n}\right)=x^{\alpha+\beta} \partial_{n} * \partial_{n} * \varphi^{\prime}\left(x_{n}\right) \\
=X_{\alpha+\beta, n} \varphi^{\prime}\left(\partial_{n} * x_{n}\right)=X_{\alpha+\beta, n} \varphi^{\prime}(1)=0
\end{gathered}
$$

$\left(\right.$ так как $\left.\varphi^{\prime}(1) \in K\right)$.

Утверждение 2) следует из утверждения 1). Утверждение 3) следует из утверждения 1) и леммы 3.19. Утверждение 4) следует из утверждения 1). Предложение доказано.

3.9. Мономиальные подпространства алгебры многочленов $P_{n}$. Пусть $S$ - подмножество из $\mathbb{N}^{n}$; векторное пространство $P_{n}(S):=\bigoplus_{\alpha \in S} K x^{\alpha}$ называется мономиалъным подпространством алгебры многочленов $P_{n}$ с ноcuтелем $S$. По определению положим $P_{n}(\varnothing):=0$. Ясно, что $\bigcap_{i \in I} P_{n}\left(S_{i}\right)=$ $P_{n}\left(\bigcap_{i \in I} S_{i}\right)$. 
ПримеР 3.22. Для каждого натурального $i=1, \ldots, n$ и ординального числа $\lambda \in\left[1, \omega^{n}\right)$ векторное пространство $\left\{p \in P_{n} \mid \frac{\partial p}{\partial x_{i}} \in P_{\lambda, n}\right\}$ является мономиальным подпространством из $P_{n}$, а потому таковым является и их пересечение

$$
\begin{aligned}
P_{\lambda, n}^{\prime}:=\left\{p \in P_{n} \mid \frac{\partial p}{\partial x_{i}} \in P_{\lambda, n}, i=1, \ldots, n\right\} & \\
& =\oplus\left\{K x^{\alpha} \mid \alpha \in \mathbb{N}^{n}, \frac{\partial x^{\alpha}}{\partial x_{i}} \in P_{\lambda, n}, i=1, \ldots, n\right\}
\end{aligned}
$$

Ясно, что $P_{\lambda, n} \subseteq P_{\lambda, n}^{\prime}$. Следующая теорема описывает векторное пространство $P_{\lambda, n}^{\prime}$ и показывает, что вложение всегда строгое и $\operatorname{dim}_{K}\left(P_{\lambda, n}^{\prime} / P_{\lambda, n}\right)<\infty$.

TeOpema 3.23. Пyсmь $\lambda \in\left[1, \omega^{n}\right)$, m.e. $\lambda=\alpha_{n} \omega^{n-1}+\alpha_{n-1} \omega^{n-2}+\cdots$ $\cdots+\alpha_{i} \omega^{i-1}+\cdots+\alpha_{j} \omega^{j-1}$, где $\alpha_{i} \in \mathbb{N}, \alpha_{j} \neq 0 u j \geqslant 1$. Тогда:

1) $P_{\lambda, n}^{\prime}=P_{\lambda, n} \oplus \bigoplus_{i=j}^{n} K \theta_{i}$, где $\theta_{i}=\left\{\begin{array}{lll}x^{\alpha}=\prod_{k=1}^{n} x_{k}^{\alpha_{k}}, & \text { если } \quad i=j, \\ x_{i} \prod_{k=i}^{n} x_{k}^{\alpha_{k}}, & \text { если } j<i \leqslant n ;\end{array}\right.$

2) $1 \leqslant \operatorname{dim}_{K}\left(P_{\lambda, n}^{\prime} / P_{\lambda, n}\right)=n-j+1 \leqslant n$;

3) $\lambda$ - непредельный ординал (т.е. $\left.\alpha_{1} \neq 0\right)$, если и только если выполнено равенство $\operatorname{dim}_{K}\left(P_{\lambda, n}^{\prime} / P_{\lambda, n}\right)=n$;

4) $1 \leqslant \operatorname{dim}_{K}\left(P_{\lambda+1, n}^{\prime} / P_{\lambda, n}^{\prime}\right)=j \leqslant n-1$ и множество элементов $\left\{x_{i} x^{\alpha}+P_{\lambda, n}^{\prime} \mid\right.$ $i=1, \ldots, j\}$ - базис векторного пространства $P_{\lambda+1, n}^{\prime} / P_{\lambda, n}^{\prime}$;

5) векторные пространства $\left\{P_{\lambda, n}^{\prime} \mid \lambda \in\left[1, \omega^{n}\right)\right\}$ различнъ; в частности, если $\lambda<\mu$, mo $P_{\lambda, n}^{\prime} \mp P_{\mu, n}^{\prime}$.

ДоказАтельство. Сначала докажем утверждение 1). Пусть $R$ - правая часть равенства в формулировке утверждения 1). Тогда $P_{\lambda, n}^{\prime} \supseteq R$ (согласно лемме 3.17$)$. В частности, множество $P_{\lambda, n}^{\prime} \backslash P_{\lambda, n}$ непусто.

Случай 1: $\lambda$ - непредельный ординал, т. е. $j=1\left(\alpha_{1} \neq 0\right)$. В этом случае

$$
P_{\lambda, n}=\oplus\left\{K x^{\alpha} \mid x^{\beta} \leqslant x^{\alpha^{\prime}}\right\}
$$

(см. (3.11)), где $x^{\alpha^{\prime}}:=x_{1}^{-1} x^{\alpha}$, т. е. $\alpha_{1}^{\prime}=\alpha_{1}-1$ и $\alpha_{i}^{\prime}=\alpha_{i}$ для $i=2, \ldots, n$. Пусть $x^{\beta} \in P_{\lambda, n}^{\prime} \backslash P_{\lambda, n}$. Тогда $x^{\beta}>x^{\alpha^{\prime}}$ и существуют натуральное $i$ такое, что $1 \leqslant i \leqslant n$ и и $\beta_{j}=\alpha_{j}$ для всех $j>i$ и $\beta_{i}=\alpha_{i}^{\prime}+1$. Тогда с необходимостью $\beta_{k}=0$ для всех $k<i$ (так как $\frac{\partial x^{\beta}}{\partial x_{k}} \leqslant x^{\alpha^{\prime}}$ для всех $k<i$ ), и потому $x^{\beta}=\theta_{i}$.

Случай 2: $\lambda$ - предельный ординал, т. е. $j>1\left(\alpha_{1}=0\right)$. В этом случае

$$
P_{\lambda, n}=\oplus\left\{K x^{\gamma} \mid x^{\gamma}<x^{\alpha}\right\}
$$

(см. (3.11)). Пусть $x^{\beta} \notin P_{\lambda, n}$. Тогда $x^{\beta} \in P_{\lambda, n}^{\prime}$, если и только если $\frac{\partial x^{\beta}}{\partial x_{i}}<x^{\alpha}$ для всех $i=1, \ldots, n$ (напомним, что $P_{\lambda, n}=\oplus\left\{K x^{\gamma} \mid x^{\gamma}<x^{\alpha}\right\}$ ), т. е. тогда и только тогда, когда:

(i) $\frac{\partial x^{\beta}}{\partial x_{i}} \leqslant x^{\alpha}$ для всех $i=1, \ldots, n$;

(ii) $\frac{\partial x^{\beta}}{\partial x_{i}} \notin K^{*} x^{\alpha}$ для всех $i=1, \ldots, n$. 
Условие (i) - это случай 1 для $\lambda+1$. Значит,

$$
\begin{aligned}
P_{\lambda, n}^{\prime} & =P_{\lambda, n}^{\prime} \cap P_{\lambda+1, n}^{\prime}=P_{\lambda, n}^{\prime} \cap\left(P_{\lambda+1, n} \oplus \bigoplus_{i=1}^{n} K \theta_{i}^{\prime}\right) \\
& =P_{\lambda, n}^{\prime} \cap\left(P_{\lambda, n} \oplus K x^{\alpha} \oplus \bigoplus_{i=1}^{n} K \theta_{i}^{\prime}\right)=P_{\lambda, n} \oplus K x^{\alpha} \oplus P_{\lambda, n}^{\prime} \cap \bigoplus_{i=1}^{n} K \theta_{i}^{\prime} \\
& =P_{\lambda, n} \oplus K x^{\alpha} \oplus \bigoplus_{i=1}^{n} P_{\lambda, n}^{\prime} \cap K \theta_{i}^{\prime},
\end{aligned}
$$

где

$$
\theta_{i}^{\prime}:=\left\{\begin{array}{lll}
x_{i} \prod_{k=i}^{n} x_{k}^{\alpha_{k}}, & \text { если } & i>j, \\
x_{i} x^{\alpha}, & \text { если } & i \leqslant j .
\end{array}\right.
$$

Заметим, что $\theta_{j}=x^{\alpha}$ и $\theta_{i}^{\prime}=\theta_{i} \in P_{\lambda, n}^{\prime}$ для $j<i \leqslant n$. Условие (ii) исключает в точности элементы $\left\{\theta_{i}^{\prime} \mid i \leqslant j\right\}$, т. е. $\bigoplus_{i=1}^{n}\left(P_{\lambda, n}^{\prime} \cap K \theta_{i}^{\prime}\right)=\bigoplus_{j<i \leqslant n} K \theta_{i}^{\prime}=$ $\bigoplus_{j<i \leqslant n} K \theta_{i}$. Доказательство утверждения 1) закончено.

Утверждение 2) следует из утверждения 1). Утверждение 3) следует из утверждения 2).

Докажем утверждение 4). Ординальное число $\lambda+1-$ непредельный ординал. В силу утверждения 3$)$ имеем $\operatorname{dim}_{K}\left(P_{\lambda+1, n}^{\prime} / P_{\lambda+1, n}\right)=n$. Заметим, что $P_{\lambda, n} \subset$ $P_{\lambda+1, n} \subset P_{\lambda+1, n}^{\prime}$ и $\operatorname{dim}_{K}\left(P_{\lambda+1, n} / P_{\lambda, n}\right)=1$. В силу утверждения 1$)$ имеем

$$
\operatorname{dim}_{K}\left(P_{\lambda+1, n}^{\prime} / P_{\lambda, n}\right)=\operatorname{dim}_{K}\left(P_{\lambda+1, n}^{\prime} / P_{\lambda+1, n}\right)+\operatorname{dim}_{K}\left(P_{\lambda+1, n} / P_{\lambda, n}\right)=n+1 .
$$

Наконец, в силу утверждения 2)

$\operatorname{dim}_{K}\left(P_{\lambda+1, n}^{\prime} / P_{\lambda, n}^{\prime}\right)=\operatorname{dim}_{K}\left(P_{\lambda+1, n}^{\prime} / P_{\lambda, n}\right)-\operatorname{dim}_{K}\left(P_{\lambda, n}^{\prime} / P_{\lambda, n}\right)=n+1-(n-j+1)=j$ и $1 \leqslant j=\operatorname{dim}_{K}\left(P_{\lambda+1, n}^{\prime} / P_{\lambda, n}^{\prime}\right) \leqslant n-1$. Элементы $\theta_{i}^{\prime}:=x_{i} x^{\alpha}, i=1, \ldots, j$, являются элементами $\theta_{i}, i=1, \ldots, j$, в утверждении 1$)$, но для ординала $\lambda+1$, а не для $\lambda$. Ясно, что $\theta_{i}^{\prime} \in P_{\lambda+1, n}^{\prime} \backslash P_{\lambda, n}^{\prime}$ для $i=1, \ldots, j$. Значит, элементы $\left\{\theta_{i}^{\prime}+P_{\lambda, n} \mid i=1, \ldots, j\right\} K$-линейно независимы в векторном пространстве $P_{\lambda+1, n}^{\prime} / P_{\lambda, n}^{\prime}$, так как векторные пространства $P_{\lambda+1, n}^{\prime}$ и $P_{\lambda, n}^{\prime}-$ мономиальные пространства. Эти элементы образуют базис векторного пространства $P_{\lambda+1, n}^{\prime} / P_{\lambda, n}^{\prime}$, так как $j=\operatorname{dim}_{K}\left(P_{\lambda+1, n}^{\prime} / P_{\lambda, n}^{\prime}\right)$.

Утверждение 5) следует из утверждения 4). Теорема доказана.

Например, для всех натуральных $i$ и $j$ имеем

$P_{i \omega^{n-1}, n}^{\prime}=P_{i \omega^{n-1}, n} \oplus K x_{n}^{i}, \quad P_{i \omega^{n-1}+j, n}^{\prime}=P_{i \omega^{n-1}+j, n} \oplus K x_{1}^{j} x_{n}^{i} \oplus \bigoplus_{2 \leqslant k \leqslant n} K x_{k} x_{n}^{i}$.

ПримеР 3.24. Рассмотрим следующие условия:

$$
P_{i-1} \frac{\partial p}{\partial x_{i}} \subseteq P_{\lambda, n}, \quad i=1, \ldots, n .
$$


Для каждого натурального $i=1, \ldots, n$, элемента $\alpha \in \mathbb{N}^{i-1}$ и ординального числа $\lambda \in\left[1, \omega^{n}\right)$ векторное пространство $\left\{p \in P_{n} \mid x^{\alpha} \frac{\partial p}{\partial x_{i}} \in P_{\lambda, n}\right\}$ является мономиальным подпространством из $P_{n}$, значит, таковым является пересечение

$$
\begin{aligned}
P_{\lambda, n}^{\prime \prime}:=\left\{p \in P_{n} \mid p \text { удовлетворяет }(3.13)\right\} & \\
= & \oplus\left\{K x^{\beta} \mid \beta \in \mathbb{N}^{n}, x^{\beta} \text { удовлетворяет (3.13) }\right\} .
\end{aligned}
$$

Для всех ординальных чисел $\lambda \in\left[1, \omega^{n}\right)$

$$
P_{\lambda, n} \subseteq P_{\lambda, n}^{\prime \prime} \subseteq P_{\lambda, n}^{\prime}
$$

Первое вложение следует из того, что $P_{\lambda, n}-\mathfrak{u}_{n}$-модуль. Если $\lambda \leqslant \mu$, то $P_{\lambda, n}^{\prime \prime} \subseteq$ $P_{\mu, n}^{\prime \prime}$. Следующее следствие показывает, что данные вложения строгие, и дает $K$-базис для каждого векторного пространства $P_{\lambda, n}^{\prime \prime}$.

СлеДСТвиЕ 3.25. Пусть $\lambda \in\left[1, \omega^{n}\right)$, m. е. $\lambda=\alpha_{m} \omega^{m-1}+\alpha_{m-1} \omega^{m-2}+\cdots$ $\cdots+\alpha_{j} \omega^{j-1}$, где $\alpha_{i} \in \mathbb{N}, \alpha_{m} \neq 0, \alpha_{j} \neq 0, m \leqslant n u j \geqslant 1$. Тогда:

1) $P_{\lambda, n}^{\prime \prime}=P_{\lambda, n} \oplus K x^{\alpha}=P_{\lambda+1, n}$, где $x^{\alpha}=\prod_{k=j}^{m} x_{k}^{\alpha_{k}}$;

2) векторные пространства $\left\{P_{\lambda, n}^{\prime \prime} \mid \lambda \in\left[1, \omega^{n}\right)\right\}$ различны; в частности, если $\lambda<\mu$, mo $P_{\lambda, n}^{\prime \prime} \varsubsetneqq P_{\mu, n}^{\prime \prime}$;

3) $\operatorname{dim}_{K}\left(P_{\lambda, n}^{\prime \prime} / P_{\lambda, n}\right)=1$;

4) $\operatorname{dim}_{K}\left(P_{\lambda+1, n}^{\prime \prime} / P_{\lambda, n}^{\prime \prime}\right)=1$.

ДокАзАтельство. Утверждение 1) сразу следует из леммы 3.17 , включений $P_{\lambda, n} \subseteq P_{\lambda, n}^{\prime \prime} \subseteq P_{\lambda, n}^{\prime}$ и теоремы 3.23.

Утверждения 2)-4) следуют из утверждения 1). Следствие доказано.

\section{§ 4. Локальная конечномерность и локальная нильпотентность алгебры Ли $\mathfrak{u}_{n}$}

Цель этого параграфа - доказательство теоремы 4.2. Ключевые идеи состоят в том, чтобы использовать однорядность алгебры $\mathfrak{u}_{n}$, индукцию по ординалам $\lambda \in\left[1, \operatorname{ord}\left(\Omega_{n}\right)\right]$ и теорему 4.1 , дающую достаточные условия нильпотентности алгебры Ли.

ТЕОРема 4.1. Пусть $J-$ идеал алгебры Ли $\mathcal{G}$ такой, что факторалгебра $Л и \overline{\mathcal{G}}:=\mathcal{G} / J$ является конечномерной нильпотентной алгеброй Ли, J - нильпотентная алгебра Ли и каждьй элемент $a \in \mathcal{G}$ действует нильпотентно на $J\left(\right.$ m.е. $(\operatorname{ad} a)^{n}(J)=0$ для некоторого натурального $\left.n=n(a)\right)$. Тогда $\mathcal{G}-$ нильпотентная алгебра Ли.

ДокАЗАТЕльство. Воспользуемся индукцией по $d=\operatorname{dim}_{K}(\overline{\mathcal{G}})$. Случай $d=0$, т. е. $\mathcal{G}=J$, очевиден.

Пусть $d=1$. Это самый важный случай, так как мы сведем общий случай к нему. Тогда $\mathcal{G}=K a \oplus J$, где $a \in \mathcal{G} \backslash J . \quad$ Пусть $\delta:=\operatorname{ad}(a) \in \operatorname{Inn}(\mathcal{G})$ и $\partial:=\operatorname{ad}(J):=\{\operatorname{ad}(j): \mathcal{G} \rightarrow \mathcal{G} \mid j \in J\}$. Алгебра Ли $J$ нильпотентна, т. е.

$$
\partial^{n}(J)=\underbrace{\operatorname{ad}(J) \cdots \operatorname{ad}(J)}_{n}(J)=0
$$


для некоторого натурального $n \geqslant 1$. Для всех элементов $b \in J$

$$
[\delta, \operatorname{ad}(b)]=[\operatorname{ad}(a), \operatorname{ad}(b)]=\operatorname{ad}([a, b]) \in \partial ;
$$

отсюда следует, что $\partial \delta \subseteq \delta \partial+\partial$. По условию отображение $\delta$ действует нильпотентно на идеале $J$. Значит, увеличивая (если нужно) число $n$, можно считать, что $\delta^{n}(J)=0$. Чтобы доказать, что $\mathcal{G}$ - нильпотентная алгебра Ли, нужно показать, что $(K \delta+\partial)^{m+1}(\mathcal{G})=0$ для некоторого натурального $m$ (из $\mathcal{G}=K a \oplus J$ следует, что $\operatorname{ad}(\mathcal{G})=K \delta+\partial)$. Достаточно показать, что

$$
(K \delta+\partial)^{m}(J)=0
$$

для некоторого натурального $m$, так как $(K \delta+\partial)(\mathcal{G}) \subseteq[\mathcal{G}, \mathcal{G}]=[K a+J$, $K a+J] \subseteq J$. Докажем, что достаточно взять $m=n(n+1)$. Пусть $m=n(n+1)$. Тогда

$$
(K \delta+\partial)^{m} \subseteq \sum_{s=0}^{m} W_{s} ;
$$

здесь $W_{s}-$ множество конечных линейных комбинаций элементов $w_{s}$, где $w_{s}-$ слово длины $m$ в алфавите $\{\delta, \partial\}$, которое содержит ровно $s$ элементов $\partial$ и $m-s$ элементов $\delta$ в своем произведении. Достаточно показать, что $w_{s}(J)=0$ для всех $s=0,1, \ldots, m$.

Заметим, что $\delta(J) \subseteq J$ и $\delta \partial^{i}(J) \subseteq \partial^{i}(J)$ для всех $i \geqslant 1$. Для всех $s$ таких, что $n \leqslant s \leqslant m$, имеем $w_{s}(J) \subseteq \partial^{s}(J)=0$, так как $s \geqslant n$ и $\partial^{n}(J)=0$. Для всех $s$ таких, что $0 \leqslant s<n$, слово $w_{s}$ имеет вид $\delta^{n_{1}} \partial \delta^{n_{2}} \partial \cdots \delta^{n_{s}} \partial \delta^{n_{s+1}}$, где $n_{1}+\cdots+n_{s+1}=m-s$. Хотя бы одно из чисел $n_{i}$ не меньше $n$, так как иначе будем иметь

$$
n^{2}=m-n \leqslant m-s=n_{1}+\cdots+n_{s+1} \leqslant(s+1)(n-1) \leqslant n(n-1) .
$$

Противоречие. Тогда $\delta^{n_{i}} \partial \cdots \delta^{n_{s}} \partial \delta^{n_{s+1}}(J) \subseteq \delta^{n_{i}}(J)=0$, так как $n_{i} \geqslant n$ и $\delta^{n}(J)=0$. Значит, $w_{s}(J)=0$.

Пусть $d>1$. Алгебра Ли $\overline{\mathcal{G}}$ нильпотентна. Зафиксируем идеал, скажем, $\overline{\mathcal{J}}$ из $\overline{\mathcal{G}}$ такой, что $\operatorname{dim}_{K}(\overline{\mathcal{G}} / \overline{\mathcal{J}})=1$. Пусть $\pi: \mathcal{G} \rightarrow \overline{\mathcal{G}}, g \mapsto \bar{g}:=g+J,-$ канонический эпиморфизм алгебр Ли. Идеал $\mathcal{J}:=\pi^{-1}(\overline{\mathcal{J}})$ алгебры Ли $\mathcal{G}$ имеет коразмерность $1\left(\operatorname{dim}_{K}(\mathcal{G} / \mathcal{J})=\operatorname{dim}_{K}(\overline{\mathcal{G}} / \overline{\mathcal{J}})=1\right)$, и $J$ - идеал алгебры Ли $\mathcal{J}$. Пара $(\mathcal{J}, J)$ удовлетворяет условиям теоремы, и $\operatorname{dim}_{K}(\mathcal{J} / J)=\operatorname{dim}_{K}(\overline{\mathcal{G}})-1<d$. В силу индукции по $d$ алгебра Ли $\mathcal{J}$ нильпотентна. Теперь пара $(\mathcal{G}, \mathcal{J})$ такая же, как в случае $d=1$, рассмотренном выше. В самом деле, $\operatorname{dim}_{K}(\mathcal{G} / \mathcal{J})=1$, и элемент $a \in \mathcal{G} \backslash \mathcal{J}$ в разложении $\mathcal{G}=K a \oplus \mathcal{J}$ действует нильпотентно на $\mathcal{J}$, так как $\overline{\mathcal{G}}$ - конечномерная нильпотентная алгебра Ли. Подробнее, пусть $\delta:=\operatorname{ad}(a)$. Тогда $\delta^{s}(\mathcal{G}) \subseteq J$ для некоторого $s$, так как $\overline{\mathcal{G}}$ - конечномерная нильпотентная алгебра Ли; $\delta^{t}(J)=0$ для некоторого $t$ по условию. Отсюда следует, что $\delta^{s+t}(\mathcal{J}) \subseteq \delta^{s+t}(\mathcal{G}) \subseteq \delta^{t}(J)=0$. Значит, $\mathcal{G}$ - нильпотентная алгебра Ли. Теорема доказана.

ТЕОРЕмА 4.2. Алгебры Ли $\mathfrak{u}_{n}$ локально конечномерны и локально нильпотентны. 
ДокАЗАТЕЛьство. Необходимо показать, что подалгебра Ли $\mathcal{G}$ алгебры $\mathfrak{u}_{n}$, порожденная конечным множеством элементов, скажем, $a_{1}, \ldots, a_{\nu},-$ конечномерная нильпотентная алгебра Ли. Случай $\nu=1$ тривиален. Без ограничения общности можно считать, что $\nu \geqslant 2$, элементы $a_{i} K$-линейно независимы и

$$
\operatorname{ord}\left(a_{1}\right)<\operatorname{ord}\left(a_{2}\right)<\cdots<\operatorname{ord}\left(a_{\nu}\right)
$$

Воспользуемся индукцией по ординальному числу $\lambda:=\operatorname{ord}\left(a_{\nu}\right) \in\left[1, \operatorname{ord}\left(\Omega_{n}\right)\right]$. По определению $\lambda$ - непредельный ординал. Начальный случай $\lambda=1$ очевиден, так как $\mathcal{G}=I_{1}=K \partial_{n}$. Итак, пусть $\lambda$ - непредельный ординал такой, что $\lambda>1$; предположим, что результат выполнен для всех непредельных ординалов $\lambda^{\prime}$ таких, что $\lambda^{\prime}<\lambda$. Тогда $\lambda^{\prime \prime}:=\operatorname{ord}\left(a_{\nu-1}\right)$ - непредельный ординал такой, что $\operatorname{ord}\left(a_{i}\right) \leqslant \lambda^{\prime \prime}<\lambda$ для всех $i=1, \ldots, \nu-1$. По индукции подалгебра Ли, скажем, $V$ алгебры $\mathfrak{u}_{n}$, порожденная элементами $a_{1}, \ldots, a_{\nu-1},-$ конечномерная нильпотентная алгебра Ли. Внутреннее дифференцирование $\delta:=\operatorname{ad}\left(a_{\nu}\right)$ алгебры Ли $\mathfrak{u}_{n}$ является локально нильпотентным дифференцированием (предложение $2.1,5)$ ), и $\operatorname{dim}_{K}(V)<\infty$; отсюда следует, что $\delta^{s+1}(V)=0$ для некоторого натурального $s$. Векторное пространство $U:=V+\delta(V)+\cdots+\delta^{s}(V)$ является конечномерным $\delta$-инвариантным подпространством идеала $I_{\lambda^{\prime \prime}}$ (см. (3.3)). По индукции подалгебра Ли, скажем, $J$ алгебры $\mathfrak{u}_{n}$, порожденная $U,-$ конечномерная нильпотентная алгебра Ли. Тогда $\delta^{t}(J)=0$ для некоторого натурального $t \geqslant 1$. Ясно, что $\delta(J) \subseteq J$, так как $\delta(U) \subseteq U$ и $\delta$ - дифференцирование. Видим, что $\mathcal{G}=K a_{\nu}+J=K a_{\nu} \oplus J$ (ясно, что $\mathcal{G} \supseteq K a_{\nu}+J$; с другой стороны, $K a_{\nu}+J$ - подалгебра Ли алгебры $\mathfrak{u}_{n}$, содержащая элементы $a_{1}, \ldots, a_{\nu}$, и потому $\left.\mathcal{G} \subseteq K a_{\nu}+J\right)$. Воспользуемся следующим утверждением: napa $(\mathcal{G}, J)$ удовлетворяет условиям теоремы 4.1. Отсюда будет следовать, что $\mathcal{G}$ - нильпотентная алгебра Ли (по теореме 4.1) и $\operatorname{dim}_{K}(\mathcal{G})=1+\operatorname{dim}_{K}(J)<\infty$. Чтобы доказать утверждение, достаточно показать, что $(\delta+\partial)^{m}(J)=0$ для всех $m \gg 0$, где $\partial=\operatorname{ad}(J)$. Зафиксируем число $n$ такое, что $\delta^{n}(J)=0$ и $\partial^{n}(J)=0$. В силу схожих причин, как в доказательстве теоремы 4.1, достаточно взять $m=n(n+1)$ :

$$
(\delta+\partial)^{m}(J) \subseteq \sum_{s=0}^{n-1} \sum_{n_{1}+\cdots+n_{s+1}=m-s} \delta^{n_{1}} \partial \delta^{n_{2}} \partial \cdots \delta^{n_{s}} \partial \delta^{n_{s+1}}(J)=0 .
$$

Теорема доказана.

\section{§ 5. Проблема изоморфизма для факторалгебр алгебр Ли $\mathfrak{u}_{n}$}

Мы уже знаем, что алгебры Ли $\mathfrak{u}_{n}$ и $\mathfrak{u}_{m}$ не изоморфны при $n \neq m$. Цель этого параграфа - получить ответ на следующий

Вопрос. Пусть $I$ и $J$ - идеалы алгебр Ли $\mathfrak{u}_{n}$ и $\mathfrak{u}_{m}$ соответственно. При каких условиях алгебры Ли $\mathfrak{u}_{n} / I$ и $\mathfrak{u}_{m} / J$ изоморфны?

Сначала рассмотрим случай, когда $n=m$ (теорема 5.1), а затем из него выведем общий случай (следствие 5.2). Пусть $\omega^{0}:=1$. 
ТеОрема 5.1. 1) Пусть $I$ - идеал алгебры Ли $\mathfrak{u}_{n}$, m.е. $I=I_{\lambda}$ для некоторого $\lambda \in\left[1, \operatorname{ord}\left(\Omega_{n}\right)\right] \cup\{0\}$ (теорема 3.6), где $I_{0}:=\{0\}$. Тогда алгебры Ли $\mathfrak{u}_{n} u$ $\mathfrak{u}_{n} / I_{\lambda}$ изоморфнь, если и только если $\lambda=i \omega^{n-2}$, где $i \in \mathbb{N}$.

2) Пусть $I$ и $J$ - идеаль алгебры Ли $\mathfrak{u}_{n}$, m.е. $I=I_{\lambda} u J=I_{\mu}$ для некоторых элементов $\lambda, \mu \in\left[1, \operatorname{ord}\left(\Omega_{n}\right)\right] \cup\{0\}$ (теорема 3.6, 1)). Тогда алгебры Ли $\mathfrak{u}_{n} / I_{\lambda}$ $u \mathfrak{u} / I_{\mu}$ изоморфны, если и только если выполнено одно из следующих условий:

(a) $\lambda=i \omega^{n-2}+\nu u \mu=j \omega^{n-2}+\nu$, гдe $i, j \in \mathbb{N} u \nu \in\left[1, \omega^{n-2}\right) \cup\{0\}$;

(b) $\lambda=\omega^{n-1}+\omega^{n-2}+\cdots+\omega^{s}+i \omega^{s-2}+\nu u \mu=\omega^{n-1}+\omega^{n-2}+\cdots+\omega^{s}+j \omega^{s-2}+\nu$, где $2 \leqslant s \leqslant n-1 ; i, j \in \mathbb{N} u \nu \in\left[1, \omega^{s-2}\right) \cup\{0\} ;$

(c) $\lambda=\mu=\omega^{n-1}+\omega^{n-2}+\cdots+\omega+\varepsilon$, где $\varepsilon=0,1$.

Для натуральных чисел $n$ и $m$ таких, что $2 \leqslant n<m$, имеется естественный изоморфизм алгебр Ли

$$
\mathfrak{u}_{n} \simeq \mathfrak{u}_{m} / I_{\nu_{m n}}, \quad X_{\alpha, i} \mapsto X_{\alpha, i}+I_{\nu_{m n}}
$$

где $X_{\alpha, i} \in \mathcal{B}_{n}, \nu_{m n}:=\omega^{m-1}+\omega^{m-2}+\cdots+\omega^{n}$.

Подробнее, $I_{\nu_{m n}}=\mathfrak{u}_{m, n+1}=\bigoplus_{j=n+1}^{m} P_{j-1} \partial_{j}$.

СлеДСтвиЕ 5.2. Пусть $n$ и $m$ - натуральные числа такие, что $2 \leqslant n<m$, и пусть I и J-идеаль алгебр Ли $\mathfrak{u}_{n} u \mathfrak{u}_{m}$ соответственно. Тогда алгебры Ли $\mathfrak{u}_{n} / I$ u $\mathfrak{u}_{m} / J$ изоморфны, если и только если

$$
\begin{gathered}
(I, J) \in\left\{\left(I_{\lambda}, I_{\mu}\right) \mid \lambda=i \omega^{n-2}+\nu, \mu=\omega^{m-1}+\omega^{m-2}+\cdots+\omega^{n}+j \omega^{n-2}+\nu,\right. \\
\left.\nu \in\left[1, \omega^{n-2}\right) \cup\{0\}, i, j \in \mathbb{N}\right\} \\
\cup \bigcup_{s=2}^{n-1}\left\{\left(I_{\lambda}, I_{\mu}\right) \mid \lambda=\omega^{n-1}+\omega^{n-2}+\cdots+\omega^{s}+i \omega^{s-2}+\nu,\right. \\
\left.\mu=\omega^{m-1}+\omega^{m-2}+\cdots+\omega^{s}+j \omega^{s-2}+\nu, \nu \in\left[1, \omega^{s-2}\right) \cup\{0\}, i, j \in \mathbb{N}\right\} \\
\cup\left\{\left(I_{\lambda}, I_{\mu}\right) \mid \lambda=\omega^{n-1}+\omega^{n-2}+\cdots+\omega+\varepsilon, \mu=\omega^{m-1}+\omega^{m-2}+\cdots\right. \\
\cdots+\omega+\varepsilon, \varepsilon=0,1\} .
\end{gathered}
$$

Напомним, что $\mathfrak{u}_{n}=\mathfrak{u}_{n-1} \oplus P_{n-1} \partial_{n}$, где $\mathfrak{u}_{n-1}$ - подалгебра Ли алгебры $\mathfrak{u}_{n}$, а $P_{n-1} \partial_{n}$ - абелев идеал алгебры Ли $\mathfrak{u}_{n}$. Ранее мы ввели $K$-базис $\mathcal{B}_{n}=\left\{X_{\alpha, i}\right\}$ алгебры Ли $\mathfrak{u}_{n}$ (см. (2.1)). Определим $K$-линейное отображение $f_{n}: \mathfrak{u}_{n} \rightarrow \mathfrak{u}_{n}$ одним из двух равносильных способов:

$$
\begin{gathered}
f_{n}(u):=\left\{\begin{array}{lll}
u, & \text { если } & u \in \mathfrak{u}_{n-1}, \\
{\left[\partial_{n-1}, u\right],} & \text { если } & u \in P_{n-1} \partial_{n},
\end{array}\right. \\
f_{n}\left(X_{\alpha, i}\right):=\left\{\begin{array}{lll}
X_{\alpha, i}, & \text { если } & \alpha \in \mathbb{N}^{i-1}, i \neq n, \\
\alpha_{n-1} X_{\alpha-e_{n-1}, n}, & \text { если } & \alpha \in \mathbb{N}^{n-1}, i=n .
\end{array}\right.
\end{gathered}
$$

ЛЕмма 5.3. Для каждого натурального $i \geqslant 1 K$-линейное отображение $f_{n}^{i}$ : $\mathfrak{u}_{n} \rightarrow \mathfrak{u}_{n}$ является эпиморфизмом алгебр Ли $u \operatorname{ker}\left(f_{n}^{i}\right)=\sum_{j=0}^{i-1} P_{n-2} x_{n-1}^{j} \partial_{n}=$ $I_{i \omega^{n-2}}$.

ДокАЗАТЕЛЬСтво. По самому определению отображения $f_{n}$ имеем

$$
\operatorname{ker}\left(f_{n}^{i}\right)=\operatorname{ker}_{P_{n-1} \partial_{n}}\left(\operatorname{ad}\left(\partial_{n-1}\right)^{i}\right)=\sum_{j=0}^{i-1} P_{n-2} x_{n-1}^{j} \partial_{n}=I_{i \omega^{n-2}}
$$


Чтобы завершить доказательство леммы, достаточно показать, что $K$-линейное отображение $f_{n}$ - гомоморфизм алгебр Ли, т. е.

$$
f_{n}([u, v])=\left[f_{n}(u), f_{n}(v)\right]
$$

для всех элементов $u$ и $v$ базиса $\mathcal{B}_{n}$ алгебры Ли $\mathfrak{u}_{n}$ (см. (2.1)). Поскольку $\mathfrak{u}_{n}=\mathfrak{u}_{n-1} \oplus P_{n-1} \partial_{n}$, имеем $\mathcal{B}_{n}=\mathcal{B}_{n-1} \cup\left\{X_{\alpha, n}\right\}_{\alpha \in \mathbb{N}^{n-1}}$. Равенство (5.3) очевидно, если либо $u, v \in \mathcal{B}_{n-1}$ (так как $f_{n}(a)=a$ для всех $a \in \mathfrak{u}_{n-1}$ ), либо $u, v \in\left\{X_{\alpha, n}\right\}_{\alpha \in \mathbb{N}^{n-1}}\left(\right.$ так как $f_{n}\left(P_{n-1} \partial_{n}\right) \subseteq P_{n-1} \partial_{n}$ и $\left[P_{n-1} \partial_{n}, P_{n-1} \partial_{n}\right]=0$ ). В оставшихся случаях, когда $u \in \mathcal{B}_{n-1}$ и $v=X_{\alpha, n}$ для некоторого $\alpha \in \mathbb{N}^{n-1}$, равенство следует из следующих трех фактов: $\partial_{n-1}$ - центральный элемент алгебры Ли $\mathfrak{u}_{n-1}$ (предложение $\left.\left.2.1,6\right)\right), f_{n}\left(P_{n-1} \partial_{n}\right) \subseteq P_{n-1} \partial_{n}$ и $\left.f_{n}\right|_{P_{n-1} \partial_{n}}=\operatorname{ad}\left(\partial_{n-1}\right)$. В самом деле, применяя внутреннее дифференцирование $\delta:=\operatorname{ad}\left(\partial_{n-1}\right)$ алгебры Ли $\mathfrak{u}_{n}$ к равенству $\left[u, X_{\alpha, n}\right]=w$, где $w \in P_{n-1} \partial_{n}$, получаем требуемое равенство

$$
\begin{aligned}
f_{n}(w) & =\delta\left(\left[u, X_{\alpha, n}\right]\right)=\left[\delta(u), X_{\alpha, n}\right]+\left[u, \delta\left(X_{\alpha, n}\right)\right]=\left[0, X_{\alpha, n}\right]+\left[f_{n}(u), f_{n}\left(X_{\alpha, n}\right)\right] \\
& =\left[f_{n}(u), f_{n}\left(X_{\alpha, n}\right)\right] .
\end{aligned}
$$

СлеДСТвИЕ 5.4. Пусть $n \geqslant 3$. Идеал $P_{n-1} \partial_{n}-$ наименъший идеал $I$ алгебры Ли $\mathfrak{u}_{n}$ такой, что факторалгебра Ли $\mathfrak{u}_{n} / I$ изоморфна алгебре Ли $\mathfrak{u}_{n-1}$.

ДоказАтельство. Очевидно, что $\mathfrak{u}_{n} / P_{n-1} \partial_{n} \simeq \mathfrak{u}_{n-1}$ и $P_{n-1} \partial_{n}=I_{\omega^{n-1}}$. Предположим, что $I \neq P_{n-1} \partial_{n}$, и получим противоречие. Тогда $I=I_{\lambda}$ для некоторого $\lambda<\omega^{n-1}$. Зафиксируем натуральное $i \geqslant 1$ такое, что $\lambda<i \omega^{n-2}$. Тогда $I_{\lambda} \subset I_{i \omega^{n-2}}$. Имеется естественный эпиморфизм алгебр Ли $\mathfrak{u}_{n} / I \rightarrow \mathfrak{u}_{n} / I_{i \omega^{n-2}} ;$ поэтому u. $\operatorname{dim}\left(\mathfrak{u}_{n} / I_{i \omega^{n-2}}\right) \leqslant \mathrm{u} . \operatorname{dim}\left(\mathfrak{u}_{n} / I\right)=\mathrm{u} \cdot \operatorname{dim}\left(\mathfrak{u}_{n-1}\right)$. В силу леммы 5.3 имеем $\mathfrak{u}_{n} / I_{i \omega^{n-2}} \simeq \mathfrak{u}_{n}$; отсюда

$$
\text { u. } \operatorname{dim}\left(\mathfrak{u}_{n-1}\right) \geqslant \text { u. } \operatorname{dim}\left(\mathfrak{u}_{n} / I_{i \omega^{n-2}}\right)=\text { u. } \operatorname{dim}\left(\mathfrak{u}_{n}\right)>\text { u. } \operatorname{dim}\left(\mathfrak{u}_{n-1}\right) .
$$

Противоречие. Следствие доказано.

ДоказАтельСтво теоремы 5.1. Докажем утверждение 1). ( $\Leftarrow)$ В силу леммы 5.3 для каждого натурального $i \geqslant 1$ отображение $f_{n}^{i}: \mathfrak{u}_{n} \rightarrow \mathfrak{u}_{n}$ - эпиморфизм алгебр Ли с ядром $I_{i \omega^{n-2}}$. Значит, $\mathfrak{u}_{n} \simeq \mathfrak{u}_{n} / I_{i \omega^{n-2}}$. Импликация $(\Rightarrow)$ следует из утверждения 2).

Чтобы доказать утверждение 2 ), воспользуемся индукцией по $n \geqslant 2$. Начальный шаг $n=2$ - непосредственное следствие леммы 5.3 и классификации идеалов алгебры Ли $\mathfrak{u}_{2}$. В силу теоремы $\left.3.6,1\right)$ собственные идеалы алгебры Ли $\mathfrak{u}_{2}$ - это $I_{n}, n \geqslant 1$, и $I_{\omega}=P_{1} \partial_{2}$. В силу леммы 5.3 имеем $\mathfrak{u}_{2} / I_{n} \simeq \mathfrak{u}_{2}$ для всех $n \geqslant 1 ; \operatorname{dim}_{K}\left(\mathfrak{u}_{2} / I_{\omega}\right)=\operatorname{dim}_{K}\left(K \partial_{1}\right)=1<\operatorname{dim}_{K}\left(\mathfrak{u}_{2}\right)=\infty$. Утверждение 2) доказано.

Пусть $n>2$, и предположим, что результат справедлив для всех $n^{\prime}<n$. Напомним, что $\mathfrak{u}_{n-1} \simeq \mathfrak{u}_{n} / I_{\omega^{n-1}}, I_{\omega^{n-1}}=P_{n-1} \partial_{n}$ и и. $\operatorname{dim}\left(\mathfrak{u}_{n-1}\right)<\mathrm{u} \cdot \operatorname{dim}\left(\mathfrak{u}_{n}\right)$. Алгебра Ли $\mathfrak{u}_{n}$ - однорядная артинова алгебра Ли; отсюда следует, что таковой является каждая из ее факторалгебр Ли. Значит, если $\mathfrak{u}_{n} / I_{\lambda} \simeq \mathfrak{u}_{n} / I_{\mu}$, то u. $\operatorname{dim}\left(\mathfrak{u}_{n} / I_{\lambda}\right)=$ u. $\operatorname{dim}\left(\mathfrak{u}_{n} / I_{\mu}\right)$. 
Шаг 1: u. $\operatorname{dim}\left(\mathfrak{u}_{n} / I_{\lambda}\right)=\mathrm{u} \cdot \operatorname{dim}\left(\mathfrak{u}_{n}\right)$ для всех $\lambda<\omega^{n-1}$.

В самом деле, из $\lambda<\omega^{n-1}$ следует, что $\lambda \leqslant i \omega^{n-2}$ для некоторого $i \geqslant 1$. Тогда $\mathfrak{u}_{n} \simeq \mathfrak{u}_{n} / I_{i \omega^{n-2}}$ (лемма 5.3), и потому

$$
\text { u. } \operatorname{dim}\left(\mathfrak{u}_{n}\right) \geqslant \text { u. } \operatorname{dim}\left(\mathfrak{u}_{n} / I_{\lambda}\right) \geqslant \text { u. } \operatorname{dim}\left(\mathfrak{u}_{n} / I_{i \omega^{n-2}}\right)=\text { u. } \operatorname{dim}\left(\mathfrak{u}_{n}\right),
$$

откуда $\mathrm{u} \cdot \operatorname{dim}\left(\mathfrak{u}_{n}\right)=\mathrm{u} \cdot \operatorname{dim}\left(\mathfrak{u}_{n} / I_{\lambda}\right)$.

Предположим, что $\mathfrak{u}_{n} / I_{\lambda} \simeq \mathfrak{u}_{n} / I_{\mu}$ для некоторых $\lambda$ и $\mu$.

Шаг 2: достаточно рассмотреть случай, когда $\lambda, \mu<\omega^{n-1}$.

Если $\lambda, \mu \geqslant \omega^{n-1}$, то утверждение 2) следует после применения индукции по $n$, так как $I_{\omega^{n-1}} \subseteq I_{\lambda}, I_{\omega^{n-1}} \subseteq I_{\mu}$ и $\mathfrak{u}_{n} / I_{\omega^{n-1}} \simeq \mathfrak{u}_{n-1}$. Без ограничения общности можно считать, что $\lambda \leqslant \mu$. Случай, когда $\lambda<\omega^{n-1} \leqslant \mu$, невозможен в силу шага 1 , так как

$$
\begin{aligned}
\text { u. } \operatorname{dim}\left(\mathfrak{u}_{n} / I_{\lambda}\right) & =\text { u. } \operatorname{dim}\left(\mathfrak{u}_{n}\right)>\text { u. } \operatorname{dim}\left(\mathfrak{u}_{n-1}\right) \\
& =\text { u. } \operatorname{dim}\left(\mathfrak{u}_{n} / I_{\omega^{n-1}}\right) \geqslant \text { u. } \operatorname{dim}\left(\mathfrak{u}_{n} / I_{\mu}\right)=\text { u. } \operatorname{dim}\left(\mathfrak{u}_{n} / I_{\lambda}\right) .
\end{aligned}
$$

Противоречие. Это завершает доказательство шага 2.

Шаг 3: ввиду леммы 5.3 можно считать, что $\lambda, \mu<\omega^{n-2}$.

Идея доказательства теоремы состоит в том, что для каждой факторалгебры Ли $\mathfrak{u}_{n} / I_{\lambda}$, где $\lambda<\omega^{n-2}$, вводится инвариант относительно изоморфизмов, который различен для всех ординальных чисел $\lambda<\omega^{n-2}$. Этот инвариант это однорядная размерность некоторых идеалов алгебры Ли $\mathfrak{u}_{n} / I_{\lambda}$. Для каждого ординального числа $\nu \in\left[1, \mathrm{u} \cdot \operatorname{dim}\left(\mathfrak{u}_{n} / I_{\lambda}\right)\right]$ пусть $I_{\nu}^{\prime}-$ единственный идеал однорядной артиновой алгебры Ли $\mathfrak{u}_{n} / I_{\lambda}$ с однорядной размерностью $\nu$. Каждый идеал однорядной артиновой алгебры Ли является характеристическим идеалом. Это решающий факт, используемый в рассуждениях ниже. Ясно, что

$$
\begin{gathered}
I_{\omega^{n-2}}^{\prime} \equiv I_{\omega^{n-2}} \equiv P_{n-2} \partial_{n} \bmod I_{\lambda} \\
I_{\omega^{n-1}+\omega^{n-2}}^{\prime} \equiv I_{\omega^{n-1}+\omega^{n-2}} \equiv P_{n-2} \partial_{n-1}+P_{n-1} \partial_{n} \bmod I_{\lambda} .
\end{gathered}
$$

Пусть $\mathfrak{a}:=I_{\omega^{n-1}+\omega^{n-2}}^{\prime}$ и $\theta:=x_{n-1} \partial_{n}+I_{\lambda} \in \mathfrak{u}_{n} / I_{\lambda}$. Заметим, что $I_{\omega^{n-2}+1}^{\prime}=$ $K \theta \oplus I_{\omega^{n-2}}^{\prime}$. Для элемента $v \in \mathfrak{u}_{n} / I_{\lambda}$ пусть $\operatorname{Cen}_{\mathfrak{a}}(v):=\{a \in \mathfrak{a} \mid[a, v]=0\}$.

Шаг 4: для всех $\sigma \in \operatorname{Aut}_{K}\left(\mathfrak{u}_{n} / I_{\lambda}\right)$ и $u \in S:=I_{\omega^{n-2}+1}^{\prime} \backslash I_{\omega^{n-2}}^{\prime}$ выполнено $\operatorname{Cen}_{\mathfrak{a}}(\sigma(u))=\operatorname{Cen}_{\mathfrak{a}}(\theta)=I_{\omega^{n-1}+\lambda}^{\prime}$.

Второе равенство следует из двух следующих фактов: $\left[x_{n-1} \partial_{n}, I_{\omega^{n-1}}\right]=$ $\left[x_{n-1} \partial_{n}, P_{n-1} \partial_{n}\right]=0$ и $\left[x^{\alpha} \partial_{n-1}, x_{n-1} \partial_{n}\right]=x^{\alpha} \partial_{n}$ для всех $\alpha \in \mathbb{N}^{n-2}$.

Первое равенство следует из того, что $\sigma(u)=\xi \theta+v+I_{\lambda}$ для некоторых элементов $\xi \in K^{*}, v \in P_{n-2} \partial_{n}$ и

$$
\left[v, I_{\omega^{n-1}+\omega^{n-2}}\right] \subseteq\left[P_{n-2} \partial_{n}, P_{n-2} \partial_{n-1}+P_{n-1} \partial_{n}\right]=0 .
$$

По-другому, используя равенство $\operatorname{Cen}_{\mathfrak{a}}(\theta)=I_{\omega^{n-1}+\lambda}^{\prime}$ и то, что $I_{\omega^{n-1}+\lambda}^{\prime}$ и $\mathfrak{a}-$ характеристические идеалы алгебры Ли $\mathfrak{u}_{n} / I_{\lambda}$, видим, что

$$
I_{\omega^{n-1}+\lambda}^{\prime}=\sigma\left(I_{\omega^{n-1}+\lambda}^{\prime}\right)=\sigma\left(\operatorname{Cen}_{\mathfrak{a}}(\theta)\right)=\operatorname{Cen}_{\sigma(\mathfrak{a})}(\sigma(\theta))=\operatorname{Cen}_{\mathfrak{a}}(\sigma(\theta)) .
$$


Поскольку $\sigma(\mathfrak{a})=\mathfrak{a}$ и $\sigma(S)=S$ для всех автоморфизмов $\sigma \in \operatorname{Aut}_{K}\left(\mathfrak{u}_{n} / I_{\lambda}\right)$, шаг 4 означает, что ординальное число

$$
\omega^{n-1}+\lambda=u \cdot \operatorname{dim}\left(I_{\omega^{n-1}+\lambda}^{\prime}\right)=u \cdot \operatorname{dim}\left(\operatorname{Cen}_{\mathfrak{a}}(\sigma(u))\right)
$$

является инвариантом относительно изоморфизмов для алгебры $\mathfrak{u}_{n} / I_{\lambda}$, где u. $\operatorname{dim}\left(I_{\omega^{n-1}+\lambda}\right)$ - однорядная размерность $\mathfrak{u}_{n}$-модуля $I_{\omega^{n-1}+\lambda}$. Если $\mathfrak{u}_{n} / I_{\lambda} \simeq$ $\mathfrak{u}_{n} / I_{\mu}$ для некоторых ординалов $\lambda, \mu<\omega^{n-2}$, то $\omega^{n-1}+\lambda=\omega^{n-1}+\mu$, откуда $\lambda=\mu$. Теорема доказана.

СлЕДСтвиЕ 5.5. Пусть $I_{\lambda}-$ идеал алгебры Ли $\mathfrak{u}_{n}$, әде $\lambda \in\left[1, \operatorname{ord}\left(\Omega_{n}\right)\right] \cup\{0\}$, причем $I_{0}:=0$. Тогда:

1) u. $\operatorname{dim}\left(\mathfrak{u}_{n} / I_{\lambda}\right)=u \cdot \operatorname{dim}\left(\mathfrak{u}_{n}\right)$ для всех $\lambda \in\left[1, \omega^{n-1}\right) \cup\{0\}$;

2) u. $\operatorname{dim}\left(\mathfrak{u}_{n} / I_{\lambda}\right)=\mathrm{u} \cdot \operatorname{dim}\left(\mathfrak{u}_{s}\right)$ для всех $\lambda \in\left[\omega^{n-1}+\omega^{n-2}+\cdots+\omega^{s}, \omega^{n-1}+\right.$ $\left.\omega^{n-2}+\cdots+\omega^{s-1}\right)$ u $s$ makux, чmo $2 \leqslant s \leqslant n-1$;

3) u. $\operatorname{dim}\left(\mathfrak{u}_{n} / I_{\lambda}\right)=1-\varepsilon$ для всех $\lambda=\omega^{n-1}+\omega^{n-2}+\cdots+\omega+\varepsilon$, где $\varepsilon=0,1$.

ДокАЗАтельство. Утверждение 1) - это шаг 1 в доказательстве теоремы 5.1. Утверждение 2) следует из утверждения 1) и (5.1). Утверждение 3) тривиально.

ДОКАЗАТЕЛЬСТВО СЛЕДСТВИЯ 5.2. Заметим, что однорядные размерности и. $\operatorname{dim}\left(\mathfrak{u}_{i}\right), i \geqslant 2$, различны, и если $\mathfrak{u}_{n} / I_{\lambda} \simeq \mathfrak{u}_{m} / I_{\mu}$, то u. $\operatorname{dim}\left(\mathfrak{u}_{n} / I_{\lambda}\right)=$ u. $\operatorname{dim}\left(\mathfrak{u}_{m} / I_{\mu}\right)$. Изоморфизм алгебр Ли $\mathfrak{u}_{n} \simeq \mathfrak{u}_{m} / I_{\nu_{m n}}$ (см. (5.1)) индуцирует биекцию из множества $\mathcal{J}\left(\mathfrak{u}_{n}\right)$ всех ненулевых идеалов алгебры Ли $\mathfrak{u}_{n}$ в множество $\mathcal{J}\left(\mathfrak{u}_{m}, I_{\nu_{m n}}\right)$ всех идеалов алгебры Ли $\mathfrak{u}_{m}$, которые собственным образом содержат идеал $I_{\nu_{m n}}$ :

$$
\mathcal{J}\left(\mathfrak{u}_{n}\right) \rightarrow \mathcal{J}\left(\mathfrak{u}_{m}, I_{\nu_{m n}}\right), \quad I_{\lambda} \mapsto I_{\nu_{m n}+\lambda} .
$$

Теперь следствие вытекает из теоремы 5.1, следствия 5.5 и (5.4).

\section{§6. Алгебра Ли $\mathfrak{u}_{\infty}$}

В этом параграфе подробно изучается алгебра Ли $\mathfrak{u}_{\infty}$. Многие свойства алгебры Ли $\mathfrak{u}_{\infty}$ схожи со свойствами алгебр Ли $\mathfrak{u}_{n}, n \geqslant 2$, но имеются несколько отличий. Например, алгебра Ли $\mathfrak{u}_{\infty}$ не разрешима, не артинова, но почти артинова, $\boldsymbol{u} \cdot \operatorname{dim}\left(\mathfrak{u}_{\infty}\right)=\omega^{\omega}$. Получена классификация всех идеалов алгебры Ли $\mathfrak{u}_{\infty}$ (теорема 6.2), все они - характеристические идеалы (следствие $6.4,2$ )). Дается критерий изоморфности факторалгебр Ли алгебры й (следствие 6.3).

Пусть $P_{\infty}:=\bigcup_{n \geqslant 1} P_{n}=K\left[x_{1}, x_{2}, \ldots\right]$ - алгебра многочленов от счетного числа переменных, а $A_{\infty}:=\bigcup_{n \geqslant 1} A_{n}=K\left\langle x_{1}, x_{2}, \ldots, \partial_{1}, \partial_{2}, \ldots\right\rangle$ - бесконечная алгебра Вейля. Алгебра Ли $\mathfrak{u}_{\infty}$ является подалгеброй Ли алгебры Ли $\left(A_{\infty},[\cdot, \cdot]\right)$. Алгебра многочленов $P_{\infty}$ является $A_{\infty}$-модулем. В частности, алгебра многочленов $P_{\infty}-\mathfrak{u}_{\infty}$-модуль. Алгебра Ли

$$
u_{\infty}:=\bigoplus_{i \geqslant 1} P_{i-1} \partial_{i}
$$


- прямая сумма абелевых (бесконечномерных для $i \geqslant 2$ ) подалгебр Ли $P_{i-1} \partial_{i}$. Для каждого натурального $i \geqslant 1$

$$
\mathfrak{u}_{\infty, i}:=\bigoplus_{j \geqslant i} P_{j-1} \partial_{j}
$$

- идеал алгебры Ли $\mathfrak{u}_{\infty}$ в силу (2.3). Ясно, что $\mathfrak{u}_{i, i} \subset \mathfrak{u}_{i+1, i} \subset \cdots \subset \mathfrak{u}_{\infty, i}=$ $\bigcup_{n \geqslant i} \mathfrak{u}_{n, i}$ для всех $i \geqslant 2$. Имеется строго убывающая цепочка идеалов алгебры Ли $\mathfrak{u}_{\infty}$

$$
\mathfrak{u}_{\infty, 1}=\mathfrak{u}_{\infty} \supset \mathfrak{u}_{\infty, 2} \supset \cdots \supset \mathfrak{u}_{\infty, n} \supset \cdots \supset \bigcap_{i \geqslant 1} \mathfrak{u}_{\infty, i}=0
$$

причем $\mathfrak{u}_{\infty} / \mathfrak{u}_{\infty, n+1} \simeq \mathfrak{u}_{n}$ для всех $n \geqslant 2$.

ПредЛОжениЕ 6.1. 1) Алгебра Ли $\mathfrak{u}_{\infty}$ не разрешима.

2) Алгебра Ли $\mathfrak{u}_{\infty}$ локально нильпотентна и локально конечномерна.

3) Каждъй элемент $u \in \mathfrak{u}_{\infty}$ действует локально нильпотентно на $\mathfrak{u}_{\infty}$-модуле $P_{\infty}$.

4) Цепочка ненулевых идеалов в (6.1) - ряд коммутантов алгебры Ли $\mathfrak{u}_{\infty}$, m.e. $\left(\mathfrak{u}_{\infty}\right)_{(i)}=\mathfrak{u}_{\infty, i+1}$ для всех $i \geqslant 0$.

5) Нижний центральный ряд алгебры Ли $\mathfrak{u}_{\infty}$ стабилизируется на первом шаге, m.е. $\left(\mathfrak{u}_{\infty}\right)^{(0)}=\mathfrak{u}_{\infty} u\left(\mathfrak{u}_{\infty}\right)^{(i)}=\mathfrak{u}_{\infty, 2}$ для всех $i \geqslant 1$.

6) Все внутренние дифберенцирования алгебры Ли $\mathfrak{u}_{\infty}$ являются локально нильпотентными дифберениированиями.

7) Центр $Z\left(\mathfrak{u}_{\infty}\right)$ алгебры Ли $\mathfrak{u}_{\infty}$ равен нулю. $B$ частности, с. $\operatorname{dim}\left(\mathfrak{u}_{\infty}\right)=0$.

8) Алгебры Ли $\mathfrak{u}_{\infty} u \mathfrak{u}_{n}$, где $n \geqslant 2$, не изоморфны.

9) Алгебра Ли $\mathfrak{u}_{\infty}$ содержит копию каждой нилъпотентной конечномерной алгебры Ли.

10) Пусть $a \in \mathfrak{u}_{\infty}$. Тогда внутреннее дифберениирование $\operatorname{ad}(a)$ является нильпотентным дифферениированием алгебры Ли $\mathfrak{u}_{\infty}$, если и только если $a=0$.

ДокАзАтельство. Утверждение 1) следует из утверждения 4). Утверждение 2) следует из теоремы 4.2 и того, что $\mathfrak{u}_{\infty}=\bigcup_{n \geqslant 2} \mathfrak{u}_{n}$. Утверждение 3 ) следует из предложения $2.1,4)$ и того, что $P_{\infty}=\bigcup_{n \geqslant 1} P_{n}$ и $\mathfrak{u}_{\infty}=\bigcup_{n \geqslant 2} \mathfrak{u}_{n}$.

Утверждения 4), 5) следуют из (2.3) и разложения $\mathfrak{u}_{\infty}=\bigoplus_{i \geqslant 1} P_{i-1} \partial_{i}$. Утверждение 6) следует из предложения $2.1,5)$ и того, что $\mathfrak{u}_{\infty}=\bigcup_{n \geqslant 2} \mathfrak{u}_{n}$ и $\mathfrak{u}_{2} \subseteq \mathfrak{u}_{3} \subseteq \cdots$.

Докажем утверждение 7$)$. Если $z \in Z\left(\mathfrak{u}_{\infty}\right)$, то $z \in \mathfrak{u}_{n}$ для некоторого $n$, и потому $z \in Z\left(\mathfrak{u}_{n}\right)=K \partial_{n}$ (предложение $\left.2.1,6\right)$ ). Поскольку $\left[\partial_{n}, x_{n} \partial_{n+1}\right]=\partial_{n+1}$, должно выполняться $z=0$.

Докажем утверждение 8). Алгебра Ли $\mathfrak{u}_{n}$ разрешима (предложение $\left.2.1,1\right)$ ), но алгебра Ли $\mathfrak{u}_{\infty}$ не разрешима (утверждение 1)). Значит, $\mathfrak{u}_{\infty} \not{4} \mathfrak{u}_{n}$ для всех $n \geqslant 2$. В силу предложения $2.1,7$ ) алгебры Ли $\mathfrak{u}_{n}, n \geqslant 2$, попарно неизоморфны.

Теперь докажем утверждение 9). Любая нильпотентная конечномерная алгебра Ли является подалгеброй алгебры Ли $\mathrm{UT}_{n}(K)$ для некоторого $n \geqslant 2$. Теперь результат следует из включений $\mathrm{UT}_{n}(K) \subseteq \mathfrak{u}_{n} \subseteq \mathfrak{u}_{\infty}$.

Наконец докажем утверждение 10). Достаточно показать, что если $a \neq 0$, то дифференцирование $\delta$ не нильпотентно. Предположим, что это не так для 
некоторого $a$, и получим противоречие. Элемент $a$ можно записать в виде суммы $p_{n} \partial_{n}+p_{n+1} \partial_{n+1}+\cdots$, где $p_{i} \in P_{i-1}$ для всех $i \geqslant n$ и $p_{n} \neq 0$. Ввиду изоморфизма алгебр Ли $\mathfrak{u}_{\infty} / \mathfrak{u}_{\infty, n+2} \simeq \mathfrak{u}_{n+1}$ внутреннее дифференцирование $\delta^{\prime}=\operatorname{ad}\left(p_{n} \partial_{n}+p_{n+1} \partial_{n+1}\right)$, индуцированное $\delta$, является нильпотентным дифференцированием алгебры Ли $\mathfrak{u}_{n+1}$. В силу леммы 2.2 имеем $p_{n}=0$. Противоречие.

Следующая теорема перечисляет все идеалы алгебры Ли $\mathfrak{u}_{\infty}$.

Теорема 6.2. Множество $\mathcal{J}\left(\mathfrak{u}_{\infty}\right)$ всех ненулевых идеалов алгебры Ли $\mathfrak{u}_{\infty}$ равно множеству $\left\{\mathfrak{u}_{\infty}, \mathfrak{u}_{\infty, n}, I_{\lambda, n}:=I_{\lambda}(n)+\mathfrak{u}_{\infty, n+1} \mid n \geqslant 2, \lambda \in\left[1, \omega^{n-1}\right)\right\}$, где $I_{\lambda}(n)$ - идеал $I_{\lambda}$ алгебры Ли $\mathfrak{u}_{n}$, определенный в $(3.3)$, m.e. $I_{\lambda}(n)=$ $\bigoplus_{(\alpha, n) \leqslant \lambda} K X_{\alpha, n} . \quad B$ частности, каждый ненулевой идеал I алгебры Ли $\mathfrak{u}_{\infty}$ содержит идеал $\mathfrak{u}_{\infty, n^{\prime}+1}$ для некоторого $n^{\prime} \geqslant 2$, и если $I=I_{\lambda, n}$, то имеем $n=\min \left\{n^{\prime} \geqslant 2 \mid \mathfrak{u}_{\infty, n^{\prime}+1} \subseteq I\right\}$.

ДокАЗАТЕЛЬСтво. Пусть $I$ - ненулевой идеал алгебры Ли $\mathfrak{u}_{\infty}$ и $a-$ ненулевой элемент из $I$. Тогда

$$
a=a_{l} \partial_{l}+a_{l+1} \partial_{l+1}+\cdots+a_{m} \partial_{m}=a_{l} \partial_{l}+\cdots
$$

для некоторых элементов $l \geqslant 1, a_{i} \in P_{i-1}$ и $a_{l} \neq 0$. Пусть $d-$ полная степень многочлена $a_{l}=\sum_{\alpha \in \mathbb{N}^{l-1}} \lambda_{\alpha} x^{\alpha} \in P_{l-1}$, где $\lambda_{\alpha} \in K$. Зафиксируем $\alpha=\left(\alpha_{i}\right) \in$ $\mathbb{N}^{l-1}$ такое, что $|\alpha|:=\alpha_{1}+\cdots+\alpha_{l-1}=d$. Применение $\operatorname{ad}(\partial)^{\alpha}:=\prod_{i=1}^{l-1} \operatorname{ad}\left(\partial_{i}\right)^{\alpha_{i}}$ к элементу $а$ дает элемент идеала $I$ типа $\alpha ! \lambda_{\alpha} \partial_{l}+\cdots$. Значит, без ограничения общности с самого начала можно считать, что $a_{l}=1$, т. е. $a=\partial_{l}+a_{l+1} \partial_{l+1}+\cdots$ $\cdots+a_{m} \partial_{m}$. Тогда

$$
I \ni\left[a, x_{l} \partial_{m+1}\right]=\left[\partial_{l}, x_{l} \partial_{m+1}\right]=\partial_{m+1} .
$$

Отсюда следует, что $I \supseteq\left[\partial_{m+1}, P_{s-1} \partial_{s}\right]=\left[\partial_{m+1}, P_{s-1}\right] \partial_{s}=P_{s-1} \partial_{s}$ для всех $s>m+1$. Это означает, что $\mathfrak{u}_{\infty, m+2} \subseteq I$. Рассмотрим эпиморфизм алгебр Ли (где $n=\min \left\{n^{\prime} \geqslant 2 \mid \mathfrak{u}_{\infty, n^{\prime}+1} \subseteq I\right\}$ )

$$
\pi_{n}: \mathfrak{u}_{\infty} \rightarrow \mathfrak{u}_{\infty} / \mathfrak{u}_{\infty, n+1} \simeq \mathfrak{u}_{n}, \quad u \mapsto \bar{u}:=u+\mathfrak{u}_{\infty, n+1}
$$

Образ $\pi_{n}(I)$ идеала $I$ - идеал алгебры $\mathfrak{u}_{n}$ такой, что $\pi_{n}(I) \subset \mathfrak{u}_{n, n}=P_{n-1} \partial_{n}$, по определению числа $n$. Без ограничения общности можно считать, что $I \neq$ $\mathfrak{u}_{\infty}, \mathfrak{u}_{\infty, 2}, \ldots$. Тогда $I=I_{\lambda, n}$ для некоторого $\lambda \in\left[1, \omega^{n-1}\right)$ по теореме $\left.3.6,1\right)$. Из двух очевидных фактов:

(i) $I_{\lambda, n} \supseteq I_{\mu, m}$ тогда и только тогда, когда либо $n<m$, либо, напротив, $n=m$ и $\lambda \geqslant \mu$,

(ii) $\mathfrak{u}_{\infty, n-1} \supset I_{\lambda, n} \supset \mathfrak{u}_{\infty, n}$ для всех $n \geqslant 2$, следует, что алгебра Ли $\mathfrak{u}_{\infty}$ является однорядной алгеброй Ли (т. е. для любых двух различных идеалов $I$ и $J$ из $\mathfrak{u}_{\infty}$ либо $I \subset J$, либо $\left.I \supset J\right)$. Значит, цепочка

$$
\begin{aligned}
\mathfrak{u}_{\infty}=\mathfrak{u}_{\infty, 1} \supset \cdots \supset I_{\lambda, 2} \supset \cdots \supset I_{1,2} \supset \mathfrak{u}_{\infty, 2} \supset \cdots \\
\cdots \supset \mathfrak{u}_{\infty, n} \supset \cdots \supset I_{\mu, n} \supset \cdots \supset I_{1, n} \supset \mathfrak{u}_{\infty, n+1} \supset \cdots
\end{aligned}
$$

содержит все ненулевые идеалы алгебры Ли $\mathfrak{u}_{\infty}$. Теорема доказана. 
В сочетании с теоремой 5.1 и следствием 5.2 следующее следствие дает критерий изоморфности факторалгебр Ли алгебры Ли $\mathfrak{u}_{\infty}$.

СлЕДСтвиЕ 6.3. 1) Пусть $I-$ - деал алгебры Ли $\mathfrak{u}_{\infty}$. Тогда $\mathfrak{u}_{\infty} / I \simeq \mathfrak{u}_{\infty}$, если и только если $I=0$.

2) Пусть $I$ и $J$ - ненулевье идеаль алгебрь Ли $\mathfrak{u}_{\infty} u n=\min \left\{n^{\prime} \geqslant 2 \mid\right.$ $\left.\mathfrak{u}_{\infty, n^{\prime}+1} \subseteq I, \mathfrak{u}_{\infty, n^{\prime}+1} \subseteq J\right\}\left(n<\infty\right.$, см. теорему 6.2). Тогда $\mathfrak{u}_{\infty} / I \simeq \mathfrak{u}_{\infty} / J$, если и только если $\mathfrak{u}_{n} / I^{\prime} \simeq \mathfrak{u}_{n} / J^{\prime}$, где $I^{\prime}:=I / \mathfrak{u}_{\infty, n+1}$ u $J^{\prime}:=J / \mathfrak{u}_{\infty, n+1} \subseteq \mathfrak{u}_{n}=$ $\mathfrak{u}_{\infty} / \mathfrak{u}_{\infty, n+1}$.

В отличие от алгебр Ли $\mathfrak{u}_{n}, n \geqslant 2$, никакая собственная факторалгебра Ли алгебры $\mathfrak{u}_{\infty}$ не изоморфна $\mathfrak{u}_{\infty}$.

Алгебра Ли $\mathcal{G}$ называется почти артиновой алгеброй Ли, если все ее собственные факторалгебры - артиновы алгебры Ли (т. е. для каждого ненулевого идеала $I$ алгебры Ли $\mathcal{G}$ факторалгебра $\mathcal{G} / I$ - артинова алгебра Ли).

СлЕДСТвиЕ 6.4. 1) Алгебра Ли $\mathfrak{u}_{\infty}$ является однорядной, не артиновой, не нётеровой, почти артиновой алгеброй Ли, и ее однорядная размерность ecmь u. $\operatorname{dim}\left(\mathfrak{u}_{\infty}\right)=\omega^{\omega}$.

2) Все идеалы алгебры Ли $\mathfrak{u}_{\infty}$ являются характеристическими идеалами.

ДокАзАтельство. Сначала докажем утверждение 1). Мы только что видели, что алгебра Ли $\mathfrak{u}_{\infty}$ однорядна (см. (6.3)). В силу теоремы 6.2 алгебра Ли $\mathfrak{u}_{\infty}$ не является ни артиновой, ни нётеровой, но является почти артиновой алгеброй Ли (так как все алгебры Ли $\mathfrak{u}_{n}$ артиновы; теорема $\left.3.6,2\right)$ ). В силу теоремы 6.2 каждый ненулевой идеал алгебры Ли $\mathfrak{u}_{\infty}$ содержит идеал $\mathfrak{u}_{\infty, n+1}$ для некоторого $n$, и факторалгебра $\mathfrak{u}_{\infty} / \mathfrak{u}_{\infty, n+1} \simeq \mathfrak{u}_{n}$ является однорядной артиновой алгеброй Ли, откуда

$$
\begin{aligned}
\text { u. } \operatorname{dim}\left(\mathfrak{u}_{\infty}\right)=\max \left\{\mathrm{u} \cdot \operatorname{dim}\left(\mathfrak{u}_{\infty} / \mathfrak{u}_{\infty, n+1}\right)=\mathrm{u} \cdot \operatorname{dim}\left(\mathfrak{u}_{n}\right)\right. & \\
& \left.=\omega^{n-1}+\omega^{n-2}+\cdots+\omega+1 \mid n \geqslant 2\right\}=\omega^{\omega}
\end{aligned}
$$

по теореме 3.6, 2).

Докажем утверждение 2). Идеалы $\mathfrak{u}_{\infty, n}, n \geqslant 1$, являются характеристическими идеалами, так как они образуют ряд коммутантов алгебры Ли $\mathfrak{u}_{\infty}$ $($ предложение $6.1,4)$ ). В силу теоремы 6.2 остается показать, что каждый идеал $I_{\lambda, n}-$ характеристический идеал. Пусть $\sigma$ - автоморфизм алгебры Ли $\mathfrak{u}_{\infty}$. Поскольку $\sigma\left(\mathfrak{u}_{\infty, n+1}\right)=\mathfrak{u}_{\infty, n+1}$, автоморфизм $\sigma$ индуцирует автоморфизм $\sigma_{n}$ факторалгебры Ли $\mathfrak{u}_{\infty} / \mathfrak{u}_{\infty, n+1} \simeq \mathfrak{u}_{n}$. Тогда $\sigma_{n}\left(\pi_{n}\left(I_{\lambda, n}\right)\right)=\pi_{n}\left(I_{\lambda}\right)$ в силу следствия 3.11 , где $\pi_{n}$ такое, как в (6.2). Значит, $\sigma\left(I_{\lambda, n}\right)=I_{\lambda, n}$, как и требуется.

Пусть $U_{\infty}:=U\left(\mathfrak{u}_{\infty}\right)$ - универсальная обертывающая алгебра алгебры Ли $\mathfrak{u}_{\infty}$. Цепочка алгебр Ли $\mathfrak{u}_{2} \subset \mathfrak{u}_{3} \subset \cdots \subset \mathfrak{u}_{\infty}=\bigcup_{n \geqslant 2} \mathfrak{u}_{n}$ дает цепочку универсальных обертывающих алгебр $U_{2} \subset U_{3} \subset \cdots \subset U_{\infty}=\bigcup_{n \geqslant 2} U_{n}$.

СледСтвиЕ 6.5. 1) Внутренние дифференцирования $\left\{\operatorname{ad}(u) \mid u \in \mathfrak{u}_{\infty}\right\}$ универсальной обертывающей алгебры $U_{\infty}$ алгебры Ли $\mathfrak{u}_{\infty}$ являются локально нильпотентными дифферениированиями.

2) Каждое мультипликативное подмножество $S$ из $U_{\infty}$, порожденное произвольным множеством элементов из $\mathfrak{u}_{\infty}$, является (левым и правым) множеством Оре в $U_{\infty}$. Значит, $S^{-1} U_{\infty} \simeq U_{\infty} S^{-1}$. 
ДокАзАтЕльство. Утверждения 1), 2) следуют из следствия 2.3 и того, что $U_{\infty}=\bigcup_{n \geqslant 2} U_{n}$.

\section{§ 7. Алгебра Ли $\widehat{\mathfrak{u}}_{\infty}$}

В этом параграфе изучается пополнение $\widehat{\mathfrak{u}}_{\infty}$ алгебры Ли $\mathfrak{u}_{\infty}$ относительно идеальной топологии на $\mathfrak{u}_{\infty}$. Его свойства еще дальше отклоняются от свойств алгебр Ли $\mathfrak{u}_{n}, n \geqslant 2$, и $\mathfrak{u}_{\infty}$. Например, ни одно ненулевое внутреннее дифференцирование алгебры Ли $\widehat{\mathfrak{u}}_{\infty}$ не является локально нильпотентным дифференцированием; алгебра Ли $\widehat{\mathfrak{u}}_{\infty}$ не является ни локально нильпотентной, ни локально конечномерной. Основной результат этого параграфа - классификация всех замкнутых и открытых идеалов топологической алгебры Ли $\widehat{\mathfrak{u}}_{\infty}$ (теорема $7.2,1)$ ). В результате доказано, что все открытые и все замкнутые идеалы топологической алгебры Ли $\widehat{\mathfrak{u}}_{\infty}$ являются топологически характеристическими идеалами (следствие $7.3,3)$ ).

Алгебра Ли $\mathfrak{u}_{\infty}$ - топологическая алгебра Ли, где топология - идеальная топология на $\mathfrak{u}_{\infty}$, т. е. множество $\left\{u+I \mid u \in \mathfrak{u}_{\infty}, I \in \mathcal{J}\left(\mathfrak{u}_{\infty}\right)\right\}$ является базисом идеальной топологии на $\mathfrak{u}_{\infty}$, где $\mathcal{J}\left(\mathfrak{u}_{\infty}\right)$ - множество ненулевых идеалов алгебры Ли $\mathfrak{u}_{\infty}$. Напомним, что топологическая алгебра Ли означает, что отображения $K \times \mathfrak{u}_{\infty} \rightarrow \mathfrak{u}_{\infty},(\lambda, v) \mapsto \lambda v, \mathfrak{u}_{\infty} \times \mathfrak{u}_{\infty} \rightarrow \mathfrak{u}_{\infty},(u, v) \mapsto u-v$, и $\mathfrak{u}_{\infty} \times \mathfrak{u}_{\infty} \rightarrow \mathfrak{u}_{\infty},(u, v) \mapsto[u, v]$, являются непрерывными отображениями, где топологии на $K \times \mathfrak{u}_{\infty}$ и $\mathfrak{u}_{\infty} \times \mathfrak{u}_{\infty}$ - топологии произведений, а топология на $K-$ дискретная топология. В силу теоремы 6.2 каждый ненулевой идеал алгебры Ли $\mathfrak{u}_{\infty}$ содержит идеал $\mathfrak{u}_{\infty, n}$ для некоторого $n \geqslant 1$. Значит, в определении топологии на $\mathfrak{u}_{\infty}$ вместо всех ненулевых идеалов можно взять идеалы $\left\{\mathfrak{u}_{\infty, n}\right\}_{n \geqslant 1}$.

Пополнение $\widehat{\mathfrak{u}}_{\infty}$ топологического пространства $\mathfrak{u}_{\infty}$ - это топологическая алгебра Ли

$$
\widehat{\mathfrak{u}}_{\infty}=\left\{\sum_{i \geqslant 1} a_{i} \partial_{i} \mid a_{i} \in P_{i-1}, i \geqslant 1\right\},
$$

где $\sum_{i \geqslant 1} a_{i} \partial_{i}$ - бесконечная сумма, однозначно определяемая ее коэффициентами $a_{i}$. Вложение $\mathfrak{u}_{\infty} \subseteq \widehat{\mathfrak{u}}_{\infty}$ является вложением топологических алгебр Ли, а топология на $\widehat{\mathfrak{u}}_{\infty}$ - это (по определению) наиболее сильная топология на $\widehat{\mathfrak{u}}_{\infty}$, для которой отображение $\mathfrak{u}_{\infty} \rightarrow \widehat{\mathfrak{u}}_{\infty}, a \mapsto a$, непрерывно.

Имеется строго убывающая цепочка идеалов алгебры Ли $\widehat{\mathfrak{u}}_{\infty}$

$$
\widehat{\mathfrak{u}}_{\infty, 1}=\widehat{\mathfrak{u}}_{\infty} \supset \widehat{\mathfrak{u}}_{\infty, 2} \supset \cdots \supset \widehat{\mathfrak{u}}_{\infty, n}:=\left\{\sum_{i \geqslant n} a_{i} \partial_{i} \in \widehat{\mathfrak{u}}_{\infty}\right\} \supset \cdots \supset \bigcap_{i \geqslant 1} \widehat{\mathfrak{u}}_{\infty, i}=0,
$$

причем $\widehat{\mathfrak{u}}_{\infty} / \widehat{\mathfrak{u}}_{\infty, n+1} \simeq \mathfrak{u}_{\infty} / \mathfrak{u}_{\infty, n+1} \simeq \mathfrak{u}_{n+1} / P_{n} \partial_{n+1} \simeq \mathfrak{u}_{n}$ для всех $n \geqslant 2$. Алгебру Ли $\widehat{\mathfrak{u}}_{\infty}$ можно также рассматривать как проективный предел проективной системы эпиморфизмов алгебр Ли

$$
\cdots \rightarrow \mathfrak{u}_{n+1} \rightarrow \mathfrak{u}_{n} \rightarrow \cdots \rightarrow \mathfrak{u}_{3} \rightarrow \mathfrak{u}_{2} \rightarrow 0
$$

где эпиморфизм $\mathfrak{u}_{n+1} \rightarrow \mathfrak{u}_{n}$ - это композиция естественного эпиморфизма $\mathfrak{u}_{n+1} \rightarrow \mathfrak{u}_{n+1} / P_{n} \partial_{n+1}$ и изоморфизма $\mathfrak{u}_{n+1} / P_{n} \partial_{n+1} \simeq \mathfrak{u}_{n}$. Каждый замкнутый 
идеал $\widehat{\mathfrak{u}}_{\infty, n}$ алгебры Ли $\widehat{\mathfrak{u}}_{\infty}$ является пополнением/замыканием идеала $\mathfrak{u}_{\infty, n}$ алгебры Ли $\mathfrak{u}_{\infty}$. Для каждого элемента $a \in \mathfrak{u}_{\infty}$ множество $\left\{a+\widehat{\mathfrak{u}}_{\infty, n}\right\}_{n \geqslant 1}$ является базисом всех открытых окрестностей элемента $a$. Эти множества $a+\widehat{\mathfrak{u}}_{\infty, n}$, $n \geqslant 1$, являются открытыми и замкнутыми множествами в $\widehat{\mathfrak{u}}_{\infty}$.

Алгебра многочленов $P_{\infty}=\bigcup_{n \geqslant 1} P_{n}=K\left[x_{1}, x_{2}, \ldots\right]$ является левым $\widehat{\mathfrak{u}}_{\infty}$-модулем: $a \cdot p=\sum_{i=1}^{n} a_{i} \partial_{i}(p)$ для любых $p \in P_{n}$ и $a=\sum_{i \geqslant 1} a_{i} \partial_{i} \in \widehat{\mathfrak{u}}_{\infty}$.

ПРеДЛОЖЕНИЕ 7.1. 1) Алгебра Ли $\widehat{\mathfrak{u}}_{\infty}$ не разрешима.

2) Алгебра Ли $\widehat{\mathfrak{u}}_{\infty}$ не локально нильпотентна и не локально конечномерна.

3) Каждый элемент $u \in \widehat{\mathfrak{u}}_{\infty}$ действует локально нильпотентно на $\widehat{\mathfrak{u}}_{\infty}$-модуле $P_{\infty}$.

4) Цепочка ненулевых идеалов в (7.1) - ряд коммутантов алгебры Ли $\widehat{\mathfrak{u}}_{\infty}$, m.e. $\left(\widehat{\mathfrak{u}}_{\infty}\right)_{(i)}=\widehat{\mathfrak{u}}_{\infty, i+1}$ для всех $i \geqslant 0$.

5) Нижний иентральныц ряд алгебры Ли $\widehat{\mathfrak{u}}_{\infty}$ стабилизируется на первом шаге, m.е. $\left(\widehat{\mathfrak{u}}_{\infty}\right)^{(0)}=\widehat{\mathfrak{u}}_{\infty} u\left(\widehat{\mathfrak{u}}_{\infty}\right)^{(i)}=\widehat{\mathfrak{u}}_{\infty, 2}$ для всех $i \geqslant 1$.

6) Все ненулевые внутренние дифберенцирования алгебры Ли $\widehat{\mathfrak{u}}_{\infty}$ не являются локально нильпотентными дифберенцированиями.

7) Централизатор $\operatorname{Cen}_{\widehat{\mathfrak{u}}_{\infty}}\left(\partial_{1}, \partial_{2}, \ldots\right)=\left\{\sum_{i \geqslant 1} \lambda_{i} \partial_{i} \in \widehat{\mathfrak{u}}_{\infty} \mid \lambda_{i} \in K\right\}$ является максимальной абелевой подалгеброй Ли из $\widehat{\mathfrak{u}}_{\infty}$.

8) Центр $Z\left(\widehat{\mathfrak{u}}_{\infty}\right)$ алгебры Ли $\widehat{\mathfrak{u}}_{\infty}$ равен нулю.

9) Алгебры Ли $\mathfrak{u}_{n}$, где $n \geqslant 2, \mathfrak{u}_{\infty} u \widehat{\mathfrak{u}}_{\infty}$ попарно неизоморфнъ.

ДоказАтельство. Утверждение 1) следует из утверждения 4).

Докажем утверждение 2). Пусть $a=\sum_{n \geqslant 1} \frac{x_{1}^{n}}{n !} \partial_{n}$. Тогда

$$
b_{i}:=\left(\operatorname{ad} \partial_{1}\right)^{i}(a)=\sum_{n \geqslant i} \frac{x_{1}^{n-i}}{(n-i) !} \partial_{n} \neq 0, \quad i \geqslant 1 .
$$

Следовательно, элементы $b_{1}, b_{2}, \ldots \quad K$-линейно независимы. Значит, подалгебра Ли $\mathcal{G}$ из $\widehat{\mathfrak{u}}_{\infty}$, порожденная элементами $\partial_{1}$ и $a$, не является ни нильпотентной, ни конечномерной.

Утверждение 3) следует из предложения 6.1, 3).

Докажем утверждение 4). Пусть $\delta_{i}:=\operatorname{ad}\left(\partial_{i}\right)$ для $i \geqslant 1$. Утверждение вытекает из следующих очевидных фактов: $\partial_{i} \in \widehat{\mathfrak{u}}_{\infty, i}$ и $\left[\partial_{i}, \widehat{\mathfrak{u}}_{\infty, i}\right]=\widehat{\mathfrak{u}}_{\infty, i+1}$ для всех $i \geqslant 1$. Отсюда следует, что $\left(\widehat{\mathfrak{u}}_{\infty}\right)_{(i)} \supseteq \widehat{\mathfrak{u}}_{\infty, i+1}$. Обратное вложение следует из предложения $6.1,4)$ и того, что $\widehat{\mathfrak{u}}_{\infty} / \widehat{\mathfrak{u}}_{\infty, i+1} \simeq \mathfrak{u}_{n}$.

Утверждение 5) следует из того, что $\left[\partial_{1}, \widehat{\mathfrak{u}}_{\infty}\right]=\widehat{\mathfrak{u}}_{\infty, 2}$ и $\left[\partial_{1}, \widehat{\mathfrak{u}}_{\infty, 2}\right]=\widehat{\mathfrak{u}}_{\infty, 2}$. Первое равенство дает равенство $\left[\widehat{\mathfrak{u}}_{\infty}, \widehat{\mathfrak{u}}_{\infty}\right]=\widehat{\mathfrak{u}}_{\infty, 2}$, а второе - равенства $\left(\widehat{\mathfrak{u}}_{\infty}\right)^{(i)}=$ $\widehat{\mathfrak{u}}_{\infty, 2}$ для $i \geqslant 1$.

Докажем утверждение 7). Имеем $C:=\operatorname{Cen}_{\widehat{\mathfrak{u}}_{\infty}}\left(\partial_{1}, \partial_{2}, \ldots\right)=\left\{\sum_{i \geqslant 1} a_{i} \partial_{i} \in \widehat{\mathfrak{u}}_{\infty} \mid\right.$ $a_{1} \in K, a_{i} \in \bigcap_{j=1}^{i-1} \operatorname{ker}_{P_{i-1}}\left(\partial_{j}\right)=K$ для всех $\left.i \geqslant 2\right\}$. Поскольку централизатор $C=\operatorname{Cen}_{\widehat{\mathfrak{u}}_{\infty}}(C)$ - абелева подалгебра Ли из $\widehat{\mathfrak{u}}_{\infty}$, он автоматически является максимальной абелевой подалгеброй из $\widehat{\mathfrak{u}}_{\infty}$.

Докажем утверждение 8). Пусть $z=\sum_{i \geqslant 1} \lambda_{i} \partial_{i}$ - центральный элемент алгебры Ли $\widehat{\mathfrak{u}}_{\infty}$. В силу утверждения 7) имеем $\lambda_{i} \in K$ для всех $i \geqslant 1$. Для всех $i \geqslant 1$ имеем $\lambda_{i} \partial_{i+1}=\left[z, x_{i} \partial_{i+1}\right]=0$, т. е. $z=0$. 
Теперь докажем утверждение 9). Мы уже знаем, что алгебры Ли $\mathfrak{u}_{n}$, где $n \geqslant 2$, и $\mathfrak{u}_{\infty}$ попарно неизоморфны (предложение $\left.6.1,8\right)$ ). Алгебры Ли $\mathfrak{u}_{n}$ разрешимы (предложение $2.1,1)$ ), но алгебра Ли $\widehat{\mathfrak{u}}_{\infty}$ не разрешима (утверждение 1$)$ ); отсюда следует, что $\widehat{\mathfrak{u}}_{\infty} \not 千 \mathfrak{u}_{n}$ для всех $n \geqslant 2$. Алгебра Ли $\mathfrak{u}_{\infty}$ локально нильпотентна (предложение $6.1,2)$ ), но алгебра Ли $\widehat{\mathfrak{u}}_{\infty}$ не локально нильпотентна (утверждение 2)); отсюда следует, что $\widehat{\mathfrak{u}}_{\infty} \nsucceq \mathfrak{u}_{\infty}$.

Наконец докажем утверждение 6). Поскольку центр алгебры Ли $\widehat{\mathfrak{u}}_{\infty}$ равен нулю (утверждение 8)), необходимо показать, что если $a-$ ненулевой элемент из $\widehat{\mathfrak{u}}_{\infty}$, то внутреннее дифференцирование $\delta=\operatorname{ad}(a)$ не является локально нильпотентным отображением. Запишем $a=\sum_{i \in I} p_{i} \partial_{i}=p_{n} \partial_{n}+\cdots$, где $p_{i} \in P_{i-1}$, $I=\left\{i \geqslant 1 \mid p_{i} \neq 0\right\} \neq \varnothing$ и $n$ - минимальное число в $I$. Пусть $b=\sum_{m \geqslant 0} \frac{x_{n}^{m}}{m !} \partial_{n+m}$. Тогда

$$
\delta^{i}(b)=p_{n}^{i} \sum_{m \geqslant i} \frac{x_{n}^{m-i}}{(m-i) !} \partial_{n+m} \neq 0, \quad i \geqslant 1 .
$$

Значит, $\delta$ не является нильпотентным отображением. Предложение доказано.

Следующая теорема дает классификацию всех открытых и замкнутых идеалов топологической алгебры Ли $\widehat{\mathfrak{u}}_{\infty} ;$ каждый ненулевой замкнутый идеал из $\widehat{\mathfrak{u}}_{\infty}$ открыт, и наоборот.

ТЕОрема 7.2. 1) Множество $\mathcal{J}\left(\widehat{\mathfrak{u}}_{\infty}\right)$ всех открытых идеалов алгебры Ли $\widehat{\mathfrak{u}}_{\infty}$ равно множеству всех ненулевых замкнутых идеалов алгебры Ли $\widehat{\mathfrak{u}}_{\infty}$ и равно множеству $\left\{\widehat{\mathfrak{u}}_{\infty}, \widehat{\mathfrak{u}}_{\infty, n}, I_{\lambda, n}:=I_{\lambda}(n)+\widehat{\mathfrak{u}}_{\infty, n+1} \mid n \geqslant 2, \lambda \in\left[1, \omega^{n-1}\right)\right\}$, дде $I_{\lambda}(n)-$ идеал $I_{\lambda}$ алгебры Ли $\mathfrak{u}_{n}$, определенный в $(3.3)$, m.е. $I_{\lambda}(n)=\bigoplus_{(\alpha, n) \leqslant \lambda} K X_{\alpha, n}$. $B$ частности, каждый ненулевой замкнутый идеал $I$ алгебры Ли $\widehat{\mathfrak{u}}_{\infty}$ содержит идеал $\widehat{\mathfrak{u}}_{\infty, n^{\prime}+1}$ для некоторого $n^{\prime} \geqslant 2$, причем если $I=I_{\lambda, n}$, то $n=\min \left\{n^{\prime} \geqslant 2 \mid \widehat{\mathfrak{u}}_{n^{\prime}+1} \subseteq I\right\}$.

2) Пусть $I$ - ненулевой идеал алгебры Ли $\widehat{\mathfrak{u}}_{\infty}$. Тогда $I$ - открытый идеал, если и только если I - замкнутый идеал.

3) Топологические алгебры Ли $\mathfrak{u}_{\infty} u \widehat{\mathfrak{u}}_{\infty}$ хаусдорфовы.

4) Нулевой идеал замкнутый, но не открытый идеал топологической алгебры Ли $\widehat{\mathfrak{u}}_{\infty}$.

Доказательство. Докажем утверждение 1) только для открытых идеалов. Для замкнутых оно будет следовать из утверждения 2). Каждый открытый идеал из $\widehat{\mathfrak{u}}_{\infty}$ должен быть ненулевым идеалом. Очевидно, что идеалы $\widehat{\mathfrak{u}}_{\infty}$, $\widehat{\mathfrak{u}}_{\infty, n}$ и $I_{\lambda, n}$ в утверждении 1$)$ - открытые идеалы. Пусть $I$ - открытый идеал алгебры Ли $\widehat{\mathfrak{u}}_{\infty}$, для которого $I \neq \widehat{\mathfrak{u}}_{\infty, n}$ при всех $n \geqslant 1$. Необходимо показать, что $I=I_{\lambda, n}$ для некоторых $\lambda$ и $n$ из утверждения 1$)$. Идеал $I$ содержит открытую окрестность нулевого элемента, т. е. $\widehat{\mathfrak{u}}_{\infty, n+1} \subseteq I$ для некоторого натурального $n \geqslant 2$. Можно считать, что $n=\min \left\{n^{\prime} \mid n^{\prime} \geqslant 2, \widehat{\mathfrak{u}}_{\infty, n^{\prime}+1} \subseteq I\right\}$. Ввиду изоморфизма алгебр Ли $\widehat{\mathfrak{u}}_{\infty} / \widehat{\mathfrak{u}}_{\infty, n+1} \simeq \mathfrak{u}_{n}$, теоремы $\left.3.6,1\right)$ и минимальности $n$ требуемое равенство и утверждение 1) очевидны.

Докажем утверждение 2$)$. $(\Rightarrow)$ Пусть $I$ - открытый идеал. В силу утверждения 1), верного для открытых идеалов, идеал $I$ таков, как в утверждении 1$)$. Все идеалы в утверждении 1$)$, т. е. $\widehat{\mathfrak{u}}_{\infty}, \widehat{\mathfrak{u}}_{\infty, n}$ и $I_{\lambda, n}$, очевидно, являются ненулевыми замкнутыми идеалами. Отсюда следует, что $I$ - ненулевой замкнутый 
идеал. $(\Leftarrow)$ Пусть $I$ - ненулевой замкнутый идеал в $\widehat{\mathfrak{u}}_{\infty}$. Достаточно показать, что $\widehat{\mathfrak{u}}_{\infty, n+1} \subseteq I$ для некоторого $n \geqslant 1$. Из этого будет автоматически следовать, что $I$ - открытый идеал. Идеал $\widehat{\mathfrak{u}}_{\infty, n+1}$ является замыканием идеала $\mathfrak{u}_{\infty, n+1}$ алгебры Ли $\mathfrak{u}_{\infty}$. Зафиксируем ненулевой элемент из $I$, скажем $a=\sum_{m \geqslant n} a_{m} \partial_{m}$, где $n \geqslant 1, a_{m} \in P_{m-1}$ для всех $m \geqslant n$ и $a_{n} \neq 0$. Пусть $d-$ полная степень многочлена $a_{n}=\sum_{\alpha \in \mathbb{N}^{n-1}} \lambda_{\alpha} x^{\alpha} \in P_{n-1}$, где $\lambda_{\alpha} \in K$. Зафиксируем $\alpha=\left(\alpha_{i}\right) \in \mathbb{N}^{n-1}$ такое, что $|\alpha|:=\alpha_{1}+\cdots+\alpha_{n-1}=d$. Применение $\operatorname{ad}(\partial)^{\alpha}:=\prod_{i=1}^{n-1} \operatorname{ad}\left(\partial_{i}\right)^{\alpha_{i}}$ к элементу а дает элемент идеала $I$ типа $\alpha ! \lambda_{\alpha} \partial_{n}+\cdots$. Значит, без ограничения общности можно с самого начала считать, что $a_{n}=1$, т. е. $a=\partial_{n}+a_{n+1} \partial_{n+1}+\cdots$. Для каждого $\alpha \in \mathbb{N}^{n}$ имеем

$$
I \ni\left[a, \frac{x^{\alpha+e_{n}}}{\alpha_{n}+1} \partial_{n+1}\right]=X_{\alpha, n+1},
$$

и потому $P_{n} \partial_{n+1} \subseteq I$. Для всех натуральных $l \geqslant n+1$ идеал алгебры Ли $\mathfrak{u}_{l}$, порожденный ее подпространством $P_{n} \partial_{n+1}$, равен $\bigoplus_{i=n+1}^{l} P_{i-1} \partial_{i}$. Значит, $\mathfrak{u}_{\infty, n+1}=\bigoplus_{i \geqslant n+1} P_{i-1} \partial_{i} \subseteq I$. Отсюда следует, что замыкание идеала $\mathfrak{u}_{\infty, n+1}$, которое равно $\widehat{\mathfrak{u}}_{\infty, n+1}$, принадлежит $I$, как и требуется.

Докажем утверждение 3$)$. Пусть $a=\sum_{i \geqslant 1} a_{i} \partial_{i}$ и $b=\sum_{i \geqslant 1} b_{i} \partial_{i}$ - различные элементы из $\mathfrak{u}_{\infty}$ (соответственно, $\left.\widehat{\mathfrak{u}}_{\infty}\right)$, где $a_{i}, b_{i} \in P_{i-1}$ для всех $i \geqslant 1$. Тогда $a_{n} \neq b_{n}$ для некоторого $n$, откуда $\left(a+\mathfrak{u}_{\infty, n+1}\right) \cap\left(b+\mathfrak{u}_{\infty, n+1}\right)=\varnothing$ (соответственно, $\left.\left(a+\widehat{\mathfrak{u}}_{\infty, n+1}\right) \cap\left(b+\widehat{\mathfrak{u}}_{\infty, n+1}\right)=\varnothing\right)$. Это означает, что $\mathfrak{u}_{\infty}$ и $\widehat{\mathfrak{u}}_{\infty}$ хаусдорфовы.

Утверждение 4) очевидно. Теорема доказана.

Топологическая алгебра Ли $\mathcal{G}$ называется замкнуто однорядной (соответственно, открыто однорядной), если множество всех замкнутых (соответственно, открытых) идеалов линейно упорядочено по включению. В силу теоремы $7.2,1)$ алгебра Ли $\widehat{\mathfrak{u}}_{\infty}$ является замкнуто и открыто однорядной алгеброй Ли. Значит, цепочка

$$
\begin{aligned}
\widehat{\mathfrak{u}}_{\infty}=\widehat{\mathfrak{u}}_{\infty, 1} \supset \cdots \supset I_{\lambda, 2} \supset \cdots \supset I_{1,2} \supset \widehat{\mathfrak{u}}_{\infty, 2} \supset \cdots \\
\cdots \supset \widehat{\mathfrak{u}}_{\infty, n} \supset \cdots \supset I_{\mu, n} \supset \cdots \supset I_{1, n} \supset \widehat{\mathfrak{u}}_{\infty, n+1} \supset \cdots \\
\cdots
\end{aligned}
$$

содержит все открытые и все ненулевые замкнутые идеалы алгебры Ли $\widehat{\mathfrak{u}}_{\infty}$.

Топологическая алгебра Ли $\mathcal{G}$ называется открыто артиновой алгеброй Ли (соответственно, замкнуто артиновой алгеброй Ли), если множество открытых (соответственно, замкнутых) идеалов из $\mathcal{G}$ удовлетворяет условию минимальности. Топологическая алгебра Ли $\mathcal{G}$ называется открыто нётеровой алгеброй Ли (соответственно, замкнуто нётеровой алгеброй Ли), если множество открытых (соответственно, замкнутых) идеалов из $\mathcal{G}$ удовлетворяет условию максимальности. Топологическая алгебра Ли $\mathcal{G}$ называется открыто почти артиновой алгеброй Ли (соответственно, замкнуто почти артиновой алгеброй Ли), если для всех ненулевых открытых (соответственно, замкнутых) идеалов $I$ из $\mathcal{G}$ факторалгебра Ли $\mathcal{G} / I$ - артинова алгебра Ли. Для топологической алгебры Ли $\mathcal{G}$ пусть

$$
\operatorname{Aut}_{c}(\mathcal{G}):=\operatorname{Aut}_{\text {Lie }}(\mathcal{G}) \cap \operatorname{Aut}_{\text {top }}(\mathcal{G})
$$


- ее группа автоморфизмов. Каждый элемент из $\operatorname{Aut}_{c}(\mathcal{G})$ является изоморфизмом алгебры Ли $\mathcal{G}$ и изоморфизмом топологического пространства $\mathcal{G}$. Идеал $I$ топологической алгебры Ли $\mathcal{G}$ называется топологически характеристическим идеалом, если $\sigma(I)=I$ для всех $\sigma \in \operatorname{Aut}_{c}(\mathcal{G})$.

СледСТВИЕ 7.3. 1) Топологическая алгебра Ли $\widehat{\mathfrak{u}}_{\infty}$ является открыто однорядной, замкнуто однорядной, открыто почти артиновой и замкнуто почти артиновой алгеброй Ли, которая не является ни открыто, ни замкнуто артиновой и не является ни открыто, ни замкнуто нётеровой.

2) Однорядные размерности множеств открытых и замкнутых идеалов топологической алгебры Ли $\widehat{\mathfrak{u}}_{\infty}$ совпадают и равны $\mathrm{u} \cdot \operatorname{dim}\left(\widehat{\mathfrak{u}}_{\infty}\right)=\omega^{\omega}$.

3) Все открытые/замкнутые идеалы алгебры Ли $\widehat{\mathfrak{u}}_{\infty}$ являются топологически характеристическими идеалами.

ДоказАтЕльство. Утверждение 1) следует из теоремы 7.2, 1). Утверждение 2) следует из теоремы $7.2,1$ ) и следствия $6.4,1)$.

Докажем утверждение 3 ). Идеалы $\widehat{\mathfrak{u}}_{\infty, n}, n \geqslant 1$, являются топологически характеристическими идеалами, так как они образуют ряд коммутантов алгебры Ли $\widehat{\mathfrak{u}}_{\infty}$ (предложение $\left.7.1,4\right)$ ). В силу теоремы $7.2,1$ ) остается показать, что каждый идеал $I_{\lambda, n}$ алгебры $\widehat{\mathfrak{u}}_{\infty}$ является топологически характеристическим идеалом. Пусть $\sigma \in \operatorname{Aut}_{c}\left(\widehat{\mathfrak{u}}_{\infty}\right)$. Поскольку $\sigma\left(\widehat{\mathfrak{u}}_{\infty, n+1}\right)=\widehat{\mathfrak{u}}_{\infty, n+1}$, автоморфизм $\sigma$ индуцирует автоморфизм $\sigma$ факторалгебры Ли $\widehat{\mathfrak{u}}_{\infty} / \widehat{\mathfrak{u}}_{\infty, n+1} \simeq \mathfrak{u}_{n}$. Так как $\widehat{\mathfrak{u}}_{\infty, n+1} \subseteq I_{\lambda, n}$ и каждый идеал алгебры Ли $\mathfrak{u}_{n}$ является характеристическим идеалом (следствие 3.11 ), должно выполняться $\sigma\left(I_{\lambda, n}\right)=I_{\lambda, n}$, т. е. $I_{\lambda, n}-$ топологически характеристический идеал алгебры $\widehat{\mathfrak{u}}_{\infty}$.

\section{Список литературы}

1. V.V. Bavula, The groups of automorphisms of the Lie algebras of triangular polynomial derivations, arXiv: 1304.4225 .

2. V. V. Bavula, "Every monomorphism of the Lie algebra of triangular polynomial derivations is an automorphism", C. R. Math. Acad. Sci. Paris, 350:11-12 (2012), 553-556.

3. В. В. Бавула, "Конечномерность $\mathrm{Ext}^{n}$-ов и Tor $_{n}$-ов простых модулей над одним классом алгебр", Функи. анализ и его прил., 25:3 (1991), 80-82; англ. пер.: V.V. Bavula, "Finite dimensionality of $\mathrm{Ext}^{n}$ and $\mathrm{Tor}_{n}$ of simple modules over one class of algebras", Funct. Anal. Appl., 25:3 (1991), 229-230.

4. В. В. Бавула, "Обобщенные алгебры Вейля и их представления", Алгебра и анализ, 4:1 (1992), 75-97; англ. пер.: V.V. Bavula, "Generalized Weyl algebras and their representations", St. Petersburg Math. J., 4:1 (1993), 71-92.

5. B. Rotman, G. T. Kneebone, The theory of sets and transfinite numbers, Oldbourne, London, 1966.

В. В. БАВУЛА

(V. V. BAVUlA)

Поступило в редакцию

Department of Pure Mathematics, 05.06 .2012

University of Sheffield, UK

E-mail: v.bavula@sheffield.ac.uk

Перевод с английского Е.И. Хухро 